\title{
Supporting Information: Quantifying polypeptoid conformational landscapes through integrated experiment and simulation
}

Sally Jiao ${ }^{1}$, Audra DeStefano ${ }^{1}$, Jacob I. Monroe ${ }^{1}$, Mikayla Barry ${ }^{2}$, Nicholas Sherck ${ }^{1}$, Thomas Casey $^{3}$, Rachel A. Segalman ${ }^{1,2}$, Songi Han ${ }^{1,3 *}$, M. Scott Shell ${ }^{1 *}$

${ }^{1}$ Department of Chemical Engineering, University of California, Santa Barbara, California 93106 U.S.A.

${ }^{2}$ Department of Materials, University of California, Santa Barbara, California, 93106 U.S.A.

${ }^{3}$ Department of Chemistry and Biochemistry, University of California, Santa Barbara, California, 93106 U.S.A.

*Corresponding authors. Email: songi@chem.ucsb.edu; shell@ucsb.edu 


\section{Table of Contents}

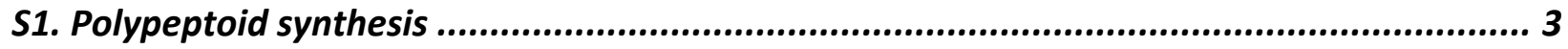

S2. Double Electron-Electron Resonance (DEER) ................................................... 9

S3. Experimental repeats .....................................................................................13

S4. Continuous-wave electron paramagnetic resonance (cw-EPR)....................................14

S5. Simulation box sizes (initial), number of water molecules, equilibration/production times, and number of independent, significant samples...................................................16

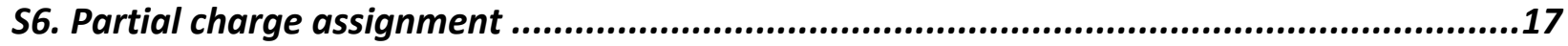

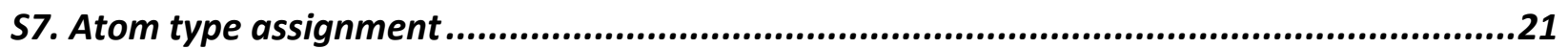

S8. Forcefield parameterization details and missing forcefield parameters...........................24

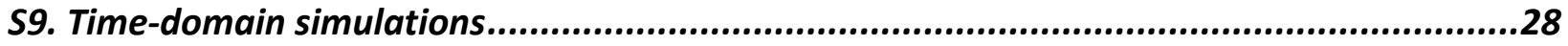

S10. Isomerization rate and correlation times of the $\omega$ dihedral .......................................30

S11. Expanded ensemble simulation methodology, validation, and convergence..................32

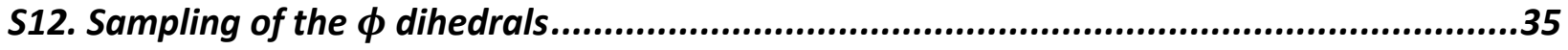

S13. Uncertainty calculations for values derived from simulations....................................36

S14. Umbrella sampling details .........................................................................38

S15. Alternative measures of the distance between distributions ....................................39

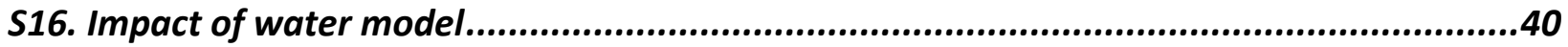

S17. Dihedral preferences for hydrophilic polypeptoid and disarcosine ...............................41

S18. Comparison of labeled and unlabeled molecules .................................................42

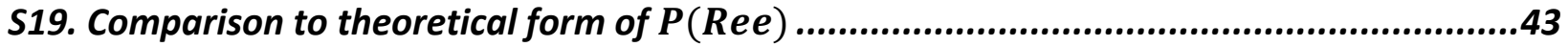

S20. Scaling of mean end-to-end distances from LongDistances........................................44

S21. Length scaling for MFTOID and modified CGenFF forcefields ...................................45

S22. Removing configurations at short distances ...................................................46

S23. Dependence of predicted distribution on CW-EPR optimal region bounds......................49

S24. Visualization of conformational distribution ..................................................51

S25. Conformational trends with increasing chain length .............................................52

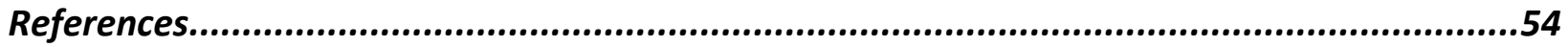




\section{S1. Polypeptoid synthesis}

All solvents and reagents are purchased from commercial suppliers and used without further purification, unless otherwise noted. Polypeptoid sequences are synthesized using an automated Prelude peptide synthesizer following previously established methods. ${ }^{1}$ Rink-amide resin (loading $0.5 \mathrm{mmol} / \mathrm{g}, 100 \mu \mathrm{mol}$ scale) is used and deprotected using 20\% 4-methylpiperdine in dimethylformamide. Bromoacetylation steps are performed for 20 minutes at room temperature with $0.4 \mathrm{M}$ bromoacetic acid and $0.4 \mathrm{M} \mathrm{N}, \mathrm{N}$ '-Diisopropylcarbodiimide in dimethylformamide. A concentration of $1 \mathrm{M}$ in dimethylformamide is used for all amines except 3-amino-1-propanol for which a concentration of $0.5 \mathrm{M}$ is used to reduce viscosity. Prior to polypeptoid synthesis, 3-amino1-propanol is protected with tert-butyldimethylsilyl chloride using an established method. ${ }^{2}$ All other amines (N-methoxyethylamine (Nme) and 4-amino TEMPO) are used as received. All monomer additions proceed for 1 hour at room temperature. The alternating hydrophilic group sequence is used instead of a homopolymer of Nme sidechains to enhance solubility in water (section S2). To summarize, we use a well-established, automated procedure to first add one spin label to a solid substrate, followed by the desired number of hydrophilic monomers, and, finally, a second spin label.

Polypeptoids are cleaved from the solid support using a cocktail of trifluoroacetic acid : water : triisopropylsilane (95: $2.5: 2.5, \mathrm{v} / \mathrm{v} / \mathrm{v})$ for 4 hours. The resin is filtered and rinsed with dichloromethane and the collected solution is dried under vacuum and lyophilized from water and acetonitrile $(1: 1, \mathrm{v} / \mathrm{v})$. Protecting groups are extracted with cyclohexane. The purified product shows a substantial decline in spin labeling due to disproportionation into oxidized and reduced species by trifluoroacetic acid during the cleavage process, so a $7 \mathrm{~N}$ ammonia in methanol : water $(9: 1)$ solution is used to regenerate the radicals for $4-12$ hours. The solution is removed under vacuum and the polypeptoid product is lyophilized from acetonitrile and water. At best, this method can only regenerate $50 \%$ of the spin labels, resulting in imperfect labeling efficiency.

One advantage of the DEER technique is that under dilute conditions, like those used in this study, only interactions between two labels on the same molecule contribute to the DEER signal. The requirement of two nearby spin labels makes DEER robust to impurities. This means that singly labeled molecules due to incomplete radical regeneration or an entire missing Ntmp unit (an impurity present in the 9mer, for example) do not distort the signal or compromise the integrity of the result. Rather, their impact is to decrease overall DEER signal relative to a solution of perfectly labeled molecules at the same concentration. All DEER signals are averaged for a sufficient time to obtain an excellent signal to noise ratio.

To confirm the presence of the target compounds, all polypeptoid samples are characterized

with matrix-assisted laser desorption/ionization (MALDI) spectrometry and liquid chromatography-mass spectrometry (LC-MS). MALDI is done on a Bruker Microflex LRF MALDI TOF mass spectrometer. Alpha-cyano matrix is prepared in 1:1 vol/vol HPLC-quality water and acetonitrile. Matrix-sample mixtures are spotted onto a polished steel MALDI target plate. Mass spectra are the average of at least 500 points collected in positive reflectron mode. LCMS is done on a Waters Acquity H-class Ultra High Pressure Liquid Chromatography system coupled with a Waters Xevo G2-XS Time-of-Flight Mass Spectrometer. All samples are dissolved in $0.1 \%$ formic acid in water and separated using a $0-60 \%$ acetonitrile gradient.

Both MALDI and LC-MS show the desired products, with some impurities. One substantial impurity is the presence of some 9mer polypeptoid with only one spin label; however, this does not impact the DEER signal because DEER requires an interaction between two spin labels. Similarly, the 15 mer shows some smaller molecules that we do not expect to impact the 
DEER measurement. Product masses are summarized in Table S1, MALDI spectra are shown in Figure S1, LC traces are shown in Figure S2, and MS spectra from LC-MS are shown in Figures S3-S6. We attribute multiple LC peaks corresponding to the same product to differences in chain conformation and/or variability in the number of regenerated free radicals.

\begin{tabular}{|l|l|c|c|}
\hline \multirow{2}{*}{ Sequence name } & Sequence $^{\mathrm{a}}$ & \multicolumn{2}{|c|}{ Exact Mass (g/mol) } \\
\cline { 3 - 4 } & & Theoretical & Observed \\
\hline 3mer-singly labeled & Ntmp-Nhp-Nme-Nhp & 573.4 & 577.3 \\
\hline 3 mer & Ntmp-Nhp-Nme-Nhp-Ntmp & 784.5 & 787.5 \\
\hline 9mer & Ntmp-(Nhp-Nme) ${ }_{4}$-Nhp-Ntmp & 1474.9 & 1474.8 \\
\hline 11 mer & Ntmp-(Nhp-Nme) 5 -Nhp-Ntmp & 1706.0 & 1706.9 \\
\hline $15 \mathrm{mer}$ & Ntmp-(Nhp-Nme) ${ }_{7}$-Nhp-Ntmp & 2165.3 & 2167.8 \\
\hline $19 \mathrm{mer}$ & Ntmp-(Nhp-Nme) ${ }_{9}$-Nhp-Ntmp & 2624.5 & 2626.3 \\
\hline
\end{tabular}

Table S1: Polypeptoid sequences with target mass and mass observed from LC-MS.

a "Ntmp" denotes the polypeptoid unit derived from 4-amino TEMPO, "Nhp" from 3-amino, 1propanol, and "Nme" from 2-methoxyethylamine.
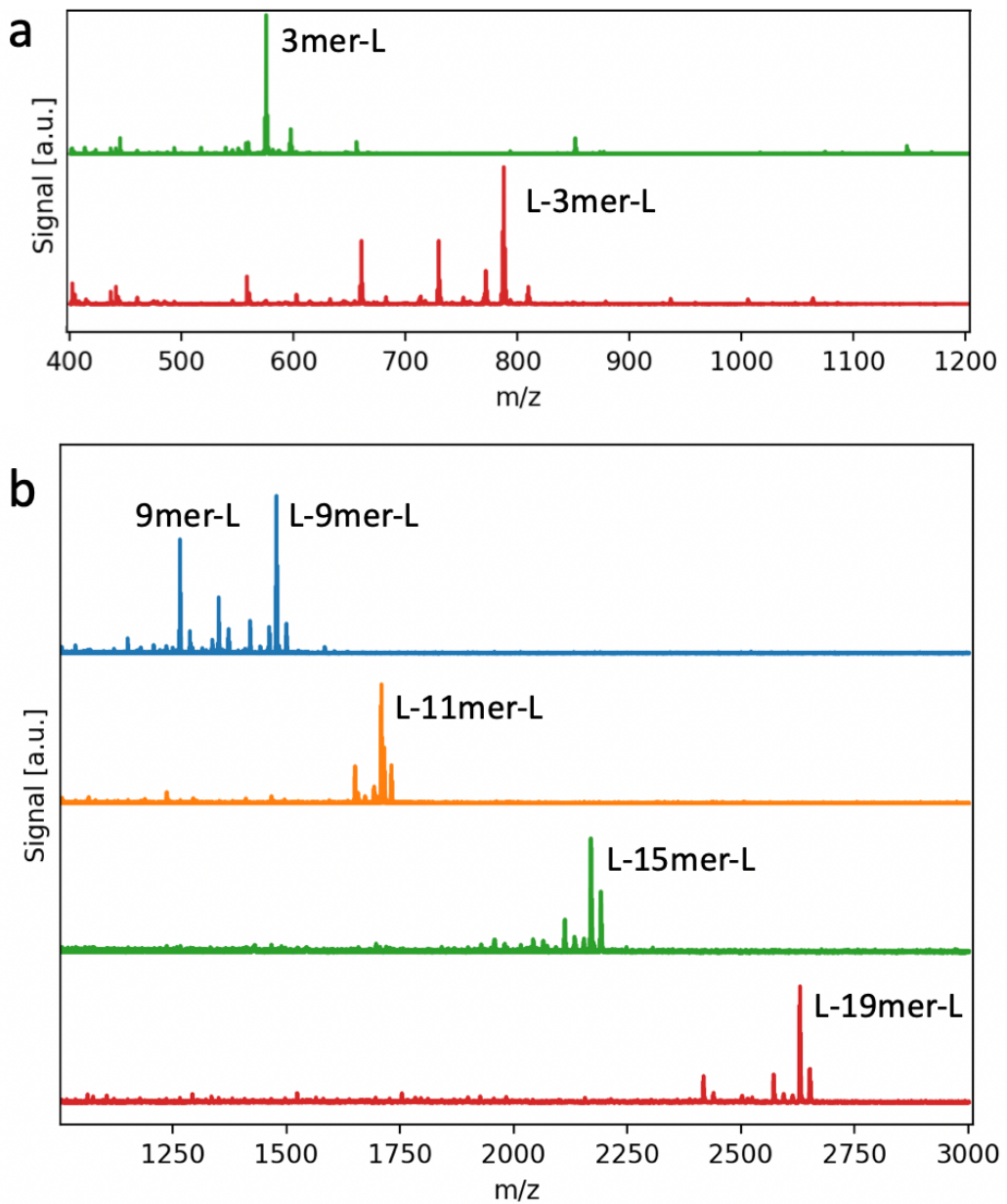

Figure S1: MALDI spectra for the (a) singly and doubly labeled 3mer (3mer-L and L-3mer-L) and the (b) 9mer, $11 \mathrm{mer}, 15 \mathrm{mer}$, and $19 \mathrm{mer}$. 


\section{a}

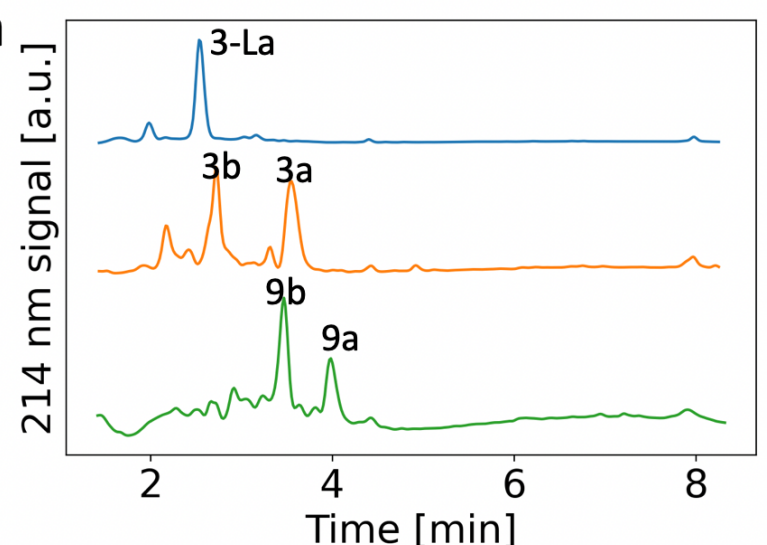

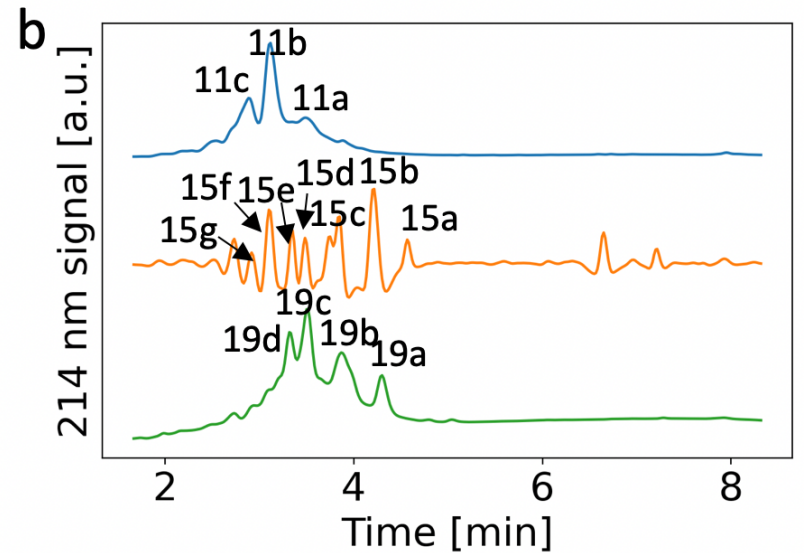

Figure S2: LC traces for the singly and doubly labeled 3mers, 9mer, $11 \mathrm{mer}, 15 \mathrm{mer}$, and $19 \mathrm{mer}$.
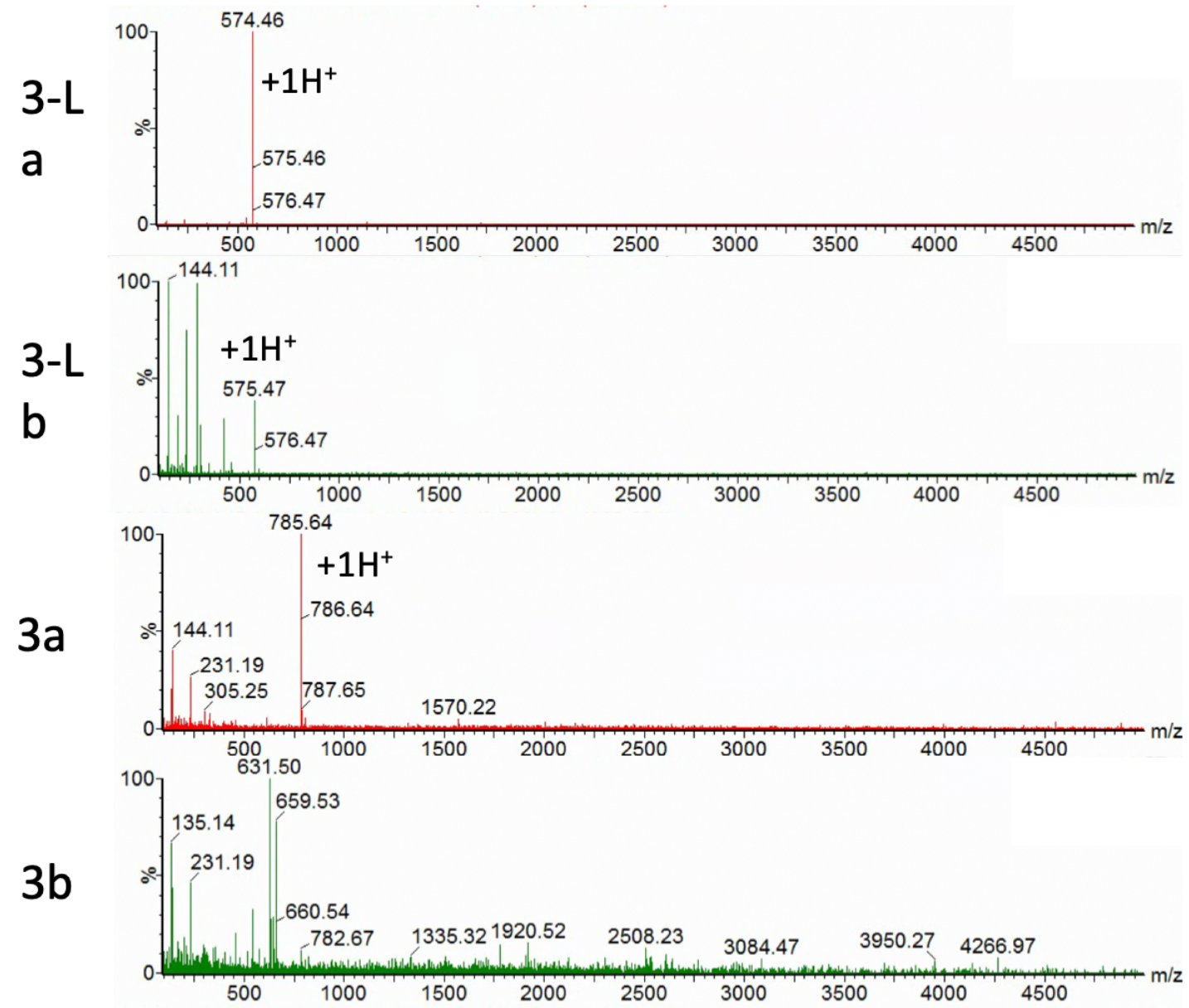

Figure S3: Mass spectra corresponding to singly (3-La, 3-Lb) and doubly (3a, 3b) 3mer LC peaks in Fig. S2. 

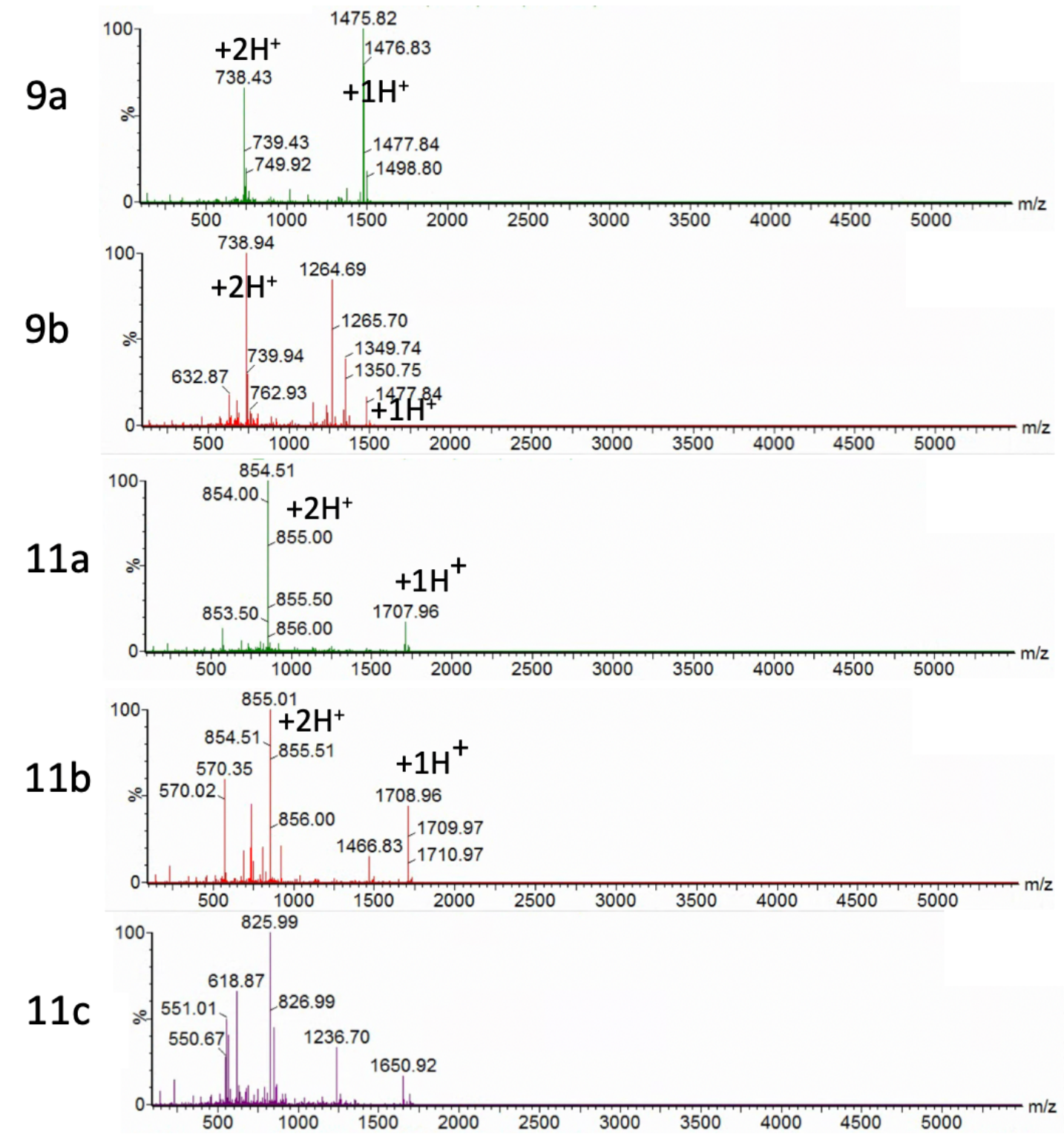

Figure S4: Mass spectra corresponding to 9mer and 11mer LC peaks in Fig. S2. 


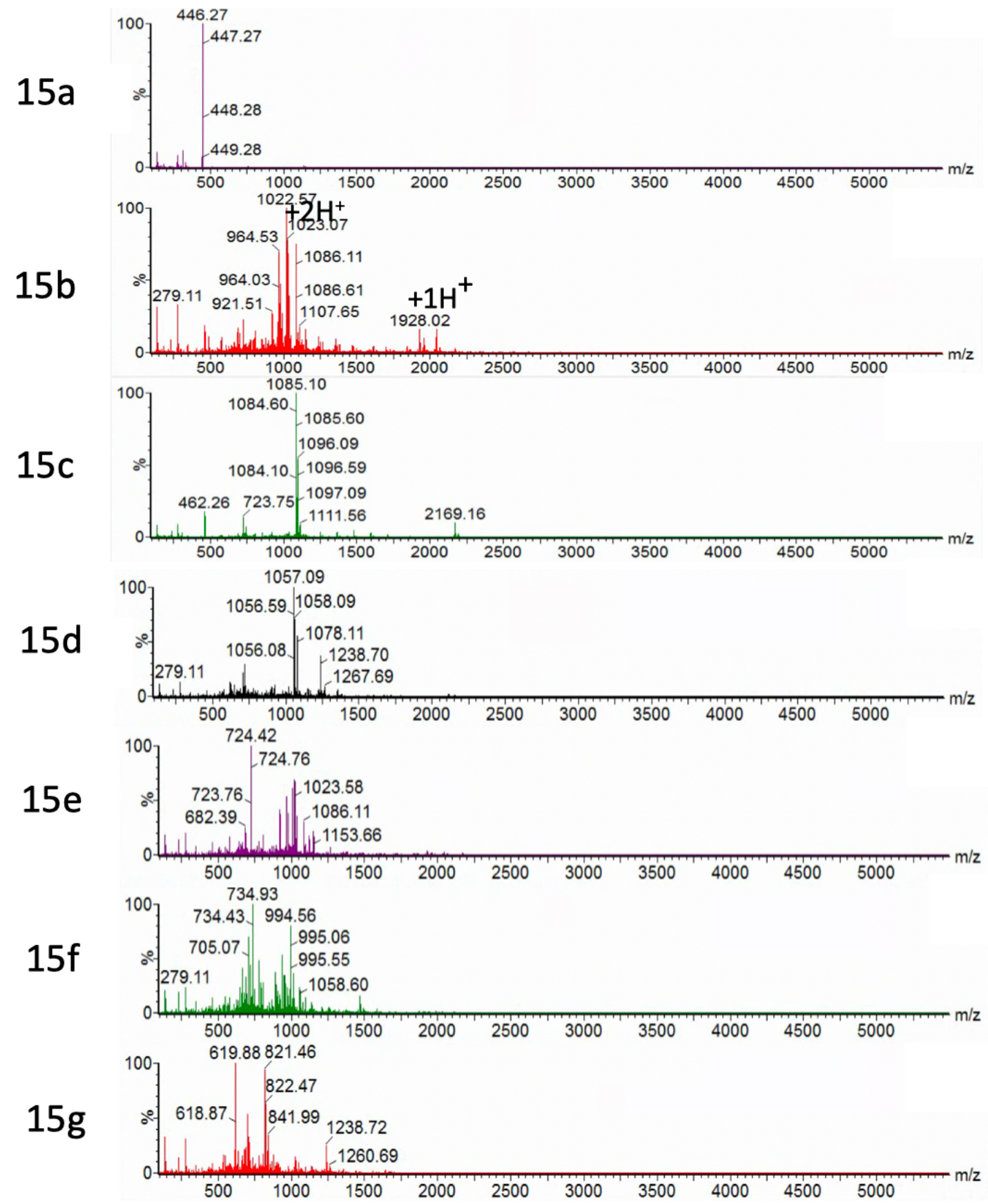

Figure S5: Mass spectra corresponding to 15 mer LC peaks in Fig. S2. We note that this sample has multiple impurities; however, none of the impurities correspond to doubly labeled molecules that contribute to DEER measurements. 

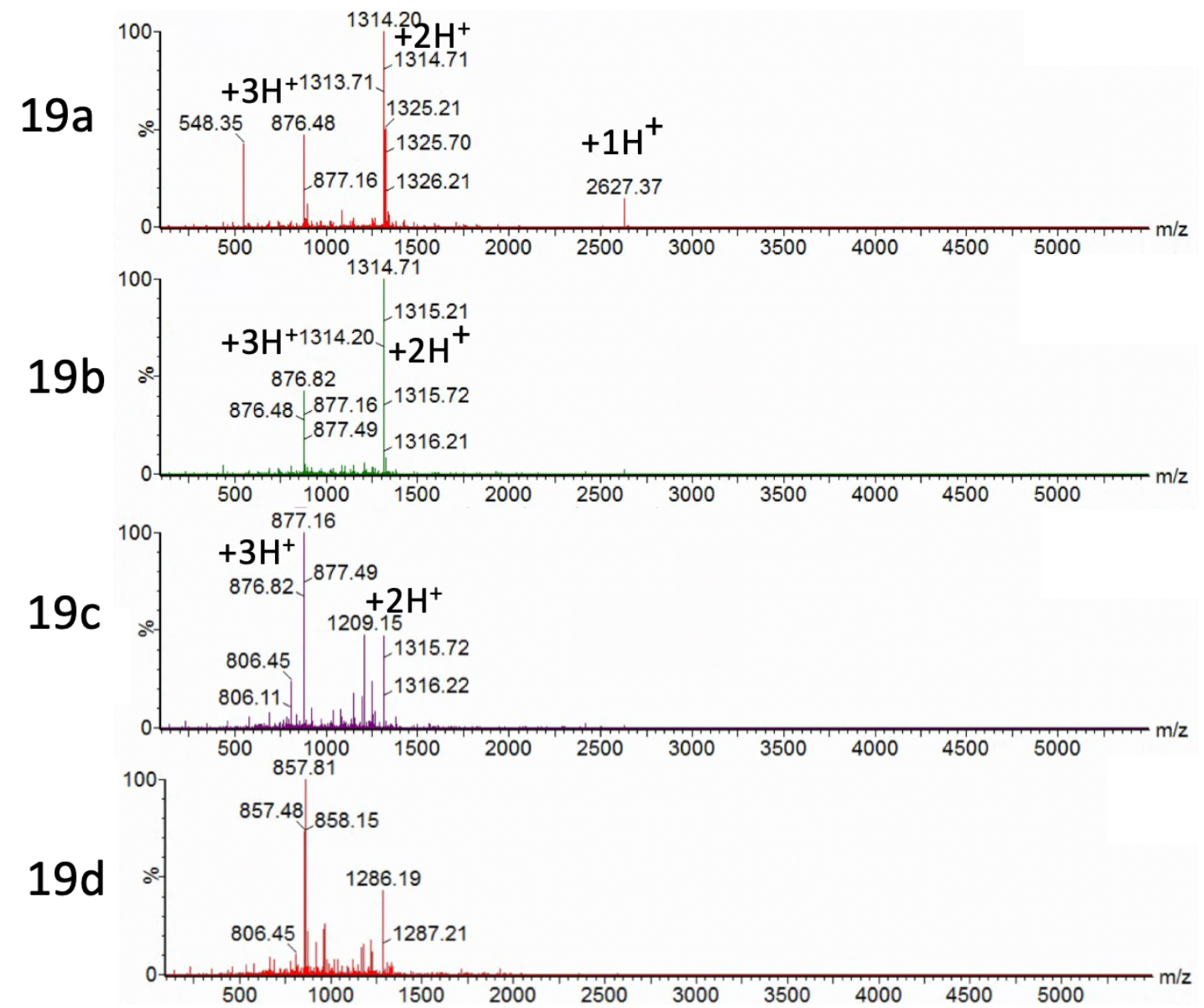

Figure S6: Mass spectra corresponding to $19 \mathrm{mer}$ LC peaks in Fig. S2. 


\section{S2. Double Electron-Electron Resonance (DEER)}

We prepare $200 \mu \mathrm{M}$ spin concentration polypeptoid samples in $\mathrm{D}_{2} \mathrm{O}$ with $30 \%$ by volume of cryoprotectant (d8-glycerol). About $40 \mu \mathrm{L}$ of sample is loaded into a $3 \mathrm{~mm}$ OD, $2 \mathrm{~mm}$ ID tube and flash frozen in liquid nitrogen to capture an ensemble average of conformations. Immediately after flash freezing, samples are loaded into a Bruker QT-II resonator in a pulsed Q-band Bruker E580 Elexsys spectrometer with a $300 \mathrm{~W}$ TWT amplifier (Applied Systems Engineering, Model $177 \mathrm{Ka}$ ); held at $65 \mathrm{~K}$ by a Bruker/ColdEdge FlexLine Cryostat (Model ER 4118HV-CF100). A four-pulse DEER sequence is used for all samples: $\pi_{\mathrm{obs}} / 2-\tau_{1}-\pi_{\mathrm{obs}}-\left(\mathrm{t}-\pi_{\mathrm{pump}}\right)-\left(\tau_{2}-\mathrm{t}\right)-\pi_{\mathrm{obs}}-$ $\tau_{2}$ - echo where subscript "obs" stands for observer pulse $\left(\omega_{A}\right.$ in Fig. 2$)$ and "pump" corresponds to $\omega_{B}$ in Fig. 2. Observer pulses are optimized using a nutation experiment and are approximately 20 seconds for a $\pi / 2$-pulse and 40 seconds for $\pi$-pulse. The $\pi_{\text {pump }}$ pulse is a linear chirp pulse with a length of $100 \mathrm{~ns}$ and a frequency sweep of $80 \mathrm{MHz}$ centered around the frequency designated as $\omega_{B}$ in Fig. 2. The observe frequency $\left(\omega_{A}\right)$ is $90 \mathrm{MHz}$ higher than the pump frequency $\left(\omega_{B}\right)$. The pulse spacing $\tau_{1}$ is $180 \mathrm{~ns}$, except for samples where deuterium electron spin echo envelope modulation (ESEEM) is appreciable, in which case a $\tau_{1}$ of $126 \mathrm{~ns}$ is used to suppress the artifact. The spacing $\tau_{1}$ is selected by $\tau_{1}=4 \pi n / \omega_{H}$ where $\omega_{H}$ is the Larmor frequency and $n$ is the whole number known to suppress electron spin echo envelope modulation. ${ }^{3}$ The spacing $\tau_{2}$ is set to $4 \mu \mathrm{s}$. Data are acquired with a $16 \mathrm{~ns}$ time resolution. Each spectrum is collected over $\sim 12 \mathrm{~h}$ resulting in averaging $\sim 150$ scans.

DEER measurements are limited fundamentally and instrumentally below $2 \mathrm{~nm}$. Briefly, the length of the pump pulse, ${ }^{4}$ limited pulse bandwidth, ${ }^{5}$ breakdown of the assumption of weak dipolar coupling, ${ }^{3,6}$ and the necessity to sample faster than the Nyquist criterion all limit DEER's resolution below $2 \mathrm{~nm}$. Ref [7] describes these limitations well.

Fig. S7 expands upon the DEER results shown in the main text by presenting the raw time domain data (Fig. S7a), $P\left(R_{e e}\right)$ 's obtained by regularization (Fig. S7b), and calculated time domain data obtained from the fitted $P\left(R_{e e}\right)$ 's (Fig. S7c-d). Regularization fits are obtained from LongDistances $^{8}$ using a three-dimensional background correction and model free fitting with a smoothing factor of 30. Uncertainties are calculated using the automated Monte Carlo error propagation method with 1 standard deviation of noise for 400 simulated spectra. Time domain data and traces calculated during the fitting procedure are shown for both LongDistances and Srivastava and Freed Singular Value Decomposition (SF-SVD) to demonstrate goodness of fit. 

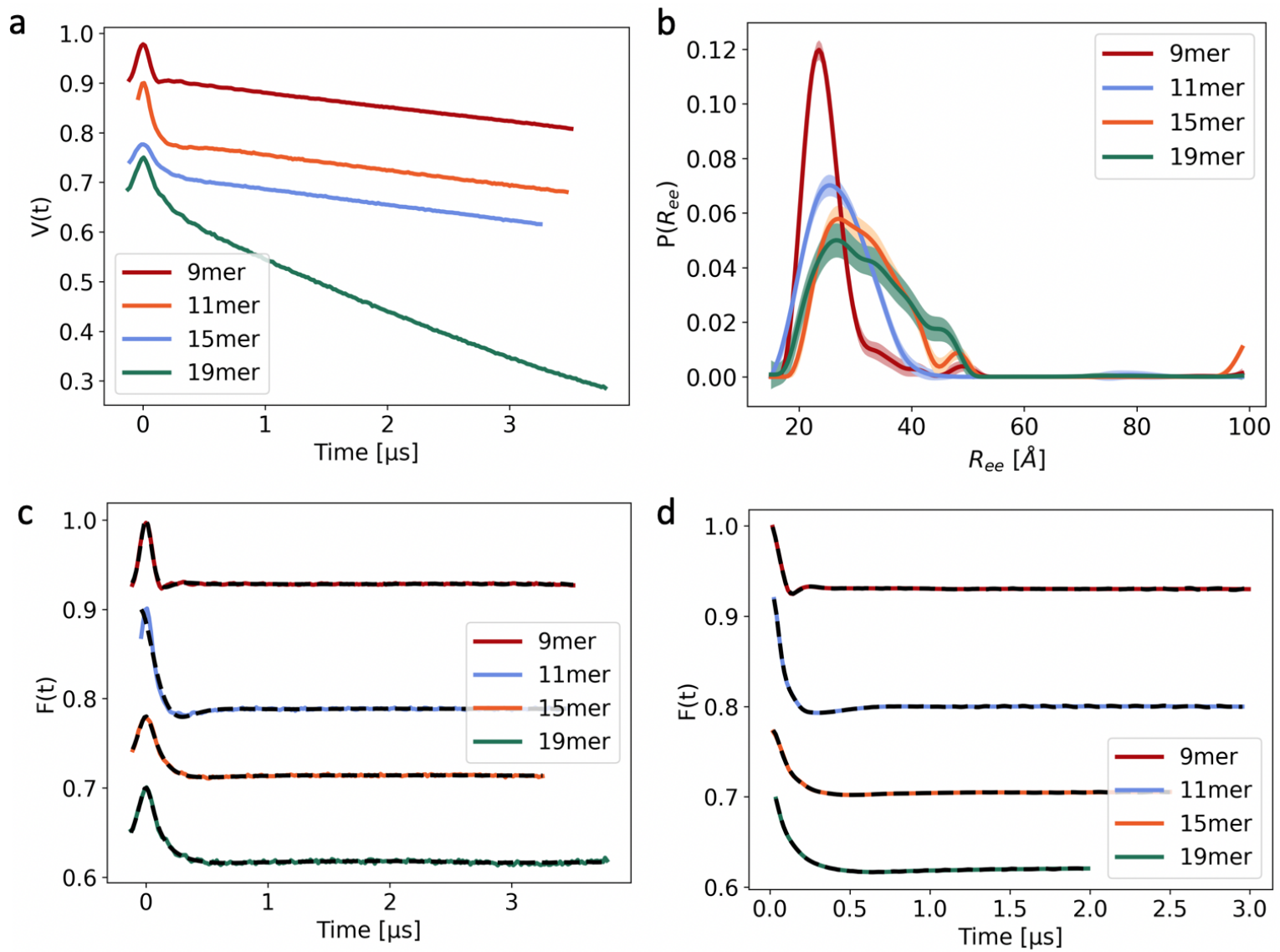

Figure S7: (a) Raw time domain data for the 9mer, 11mer, 15mer, and 19mer polypeptoid sequences. (b) Corresponding regularization $P\left(R_{e e}\right)$ 's with areas normalized to 1 . Background-corrected time domain data (colored) and calculated traces (black dashes) from (c) regularization and (d) SF-SVD.

We estimate uncertainties in the SF-SVD-fitted distance distributions by adding random, artificial noise, of equal magnitude to the noise in the raw data, to the smoothed time-domain signal, ${ }^{9}$ fitting a distance distribution, ${ }^{10}$ and repeating this a total of 20 times. We report the averaged distribution and 95\% confidence interval. The computation of uncertainties for DEERderived distance distributions, crucial for comparisons of broad and multi-featured distributions of disordered molecules, is an evolving field, but the approach used here is consistent with current best practices. ${ }^{11}$ Fig. S8 and S9 demonstrate this approach for the 9 mer and 19 mer polypeptoids considered in this study. 

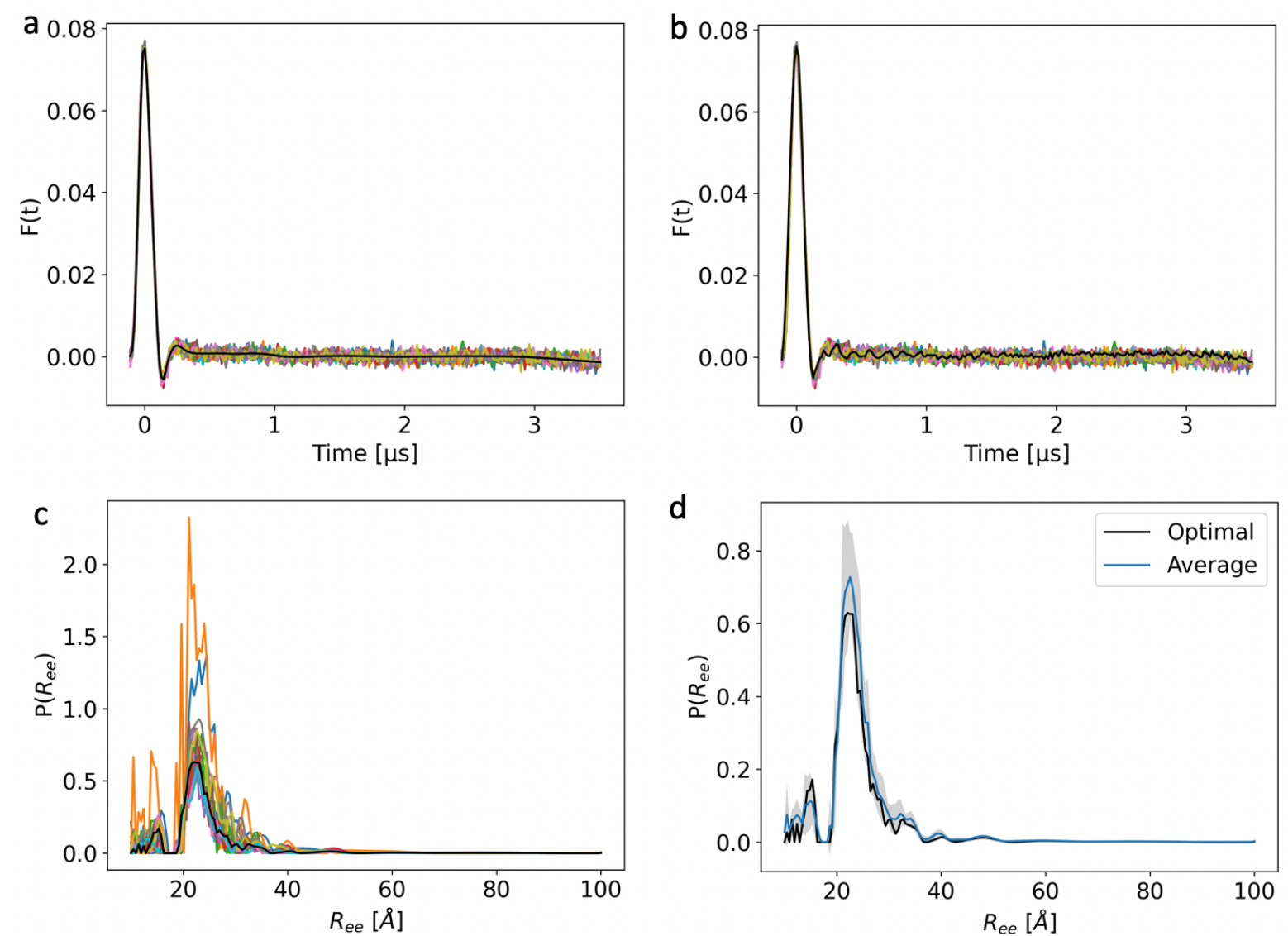

Figure S8: An example of the uncertainty calculation process is shown here for the 9mer polypeptoid. We (a) add artificial noise to the denoised data to (b) encompass that in the raw signal. Then, we (c) calculate distance distributions from the noisy spectra using SF-SVD. (d) From these noisy spectra, $95 \%$ confidence intervals are calculated. The mean distribution obtained from the noisy spectra agrees well with the optimal $P\left(R_{e e}\right)$ obtained from the smoothed spectra. 

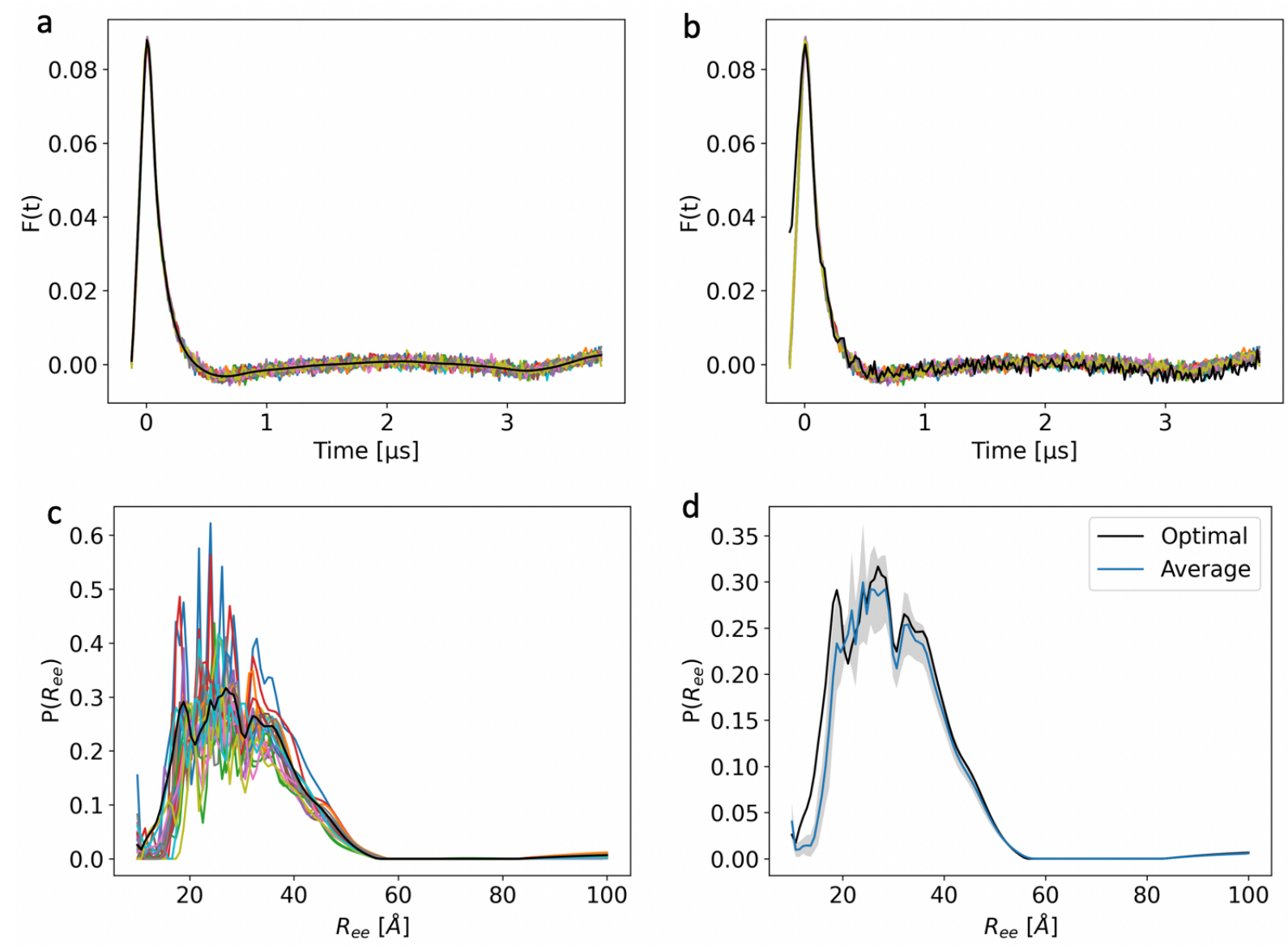

Figure S9: As for the 9mer polypeptoid, this method also works well with broader distributions, such as the $19 \mathrm{mer}$ polypeptoid that is the broadest distribution considered in this study. We (a) add artificial noise to the denoised data to (b) encompass that in the raw signal. Then, we (c) calculate distance distributions from the noisy spectra using SFSVD. (d) From these noisy spectra, 95\% confidence intervals are calculated. Just as for the narrower distribution, the mean of the distributions obtained from noisy spectra agrees well with the optimal $P\left(R_{e e}\right)$ obtained from the smoothed spectra. The uncertainty, however, increases.

The presence of two spin labels significantly decreases solubility, consistent with poor solubility of nitroxide containing polymers in water. ${ }^{12}$ The final polypeptoid concentration, 200 $\mu \mathrm{M}$, is sufficiently dilute that the effect of inter-polypeptoid interactions (e.g. aggregation) is negligible. This is demonstrated visually in Fig. S10 where a more concentrated solution appears cloudy. Concentrations of around $100 \mu \mathrm{M}$ are routinely used for DEER on proteins. ${ }^{6}$

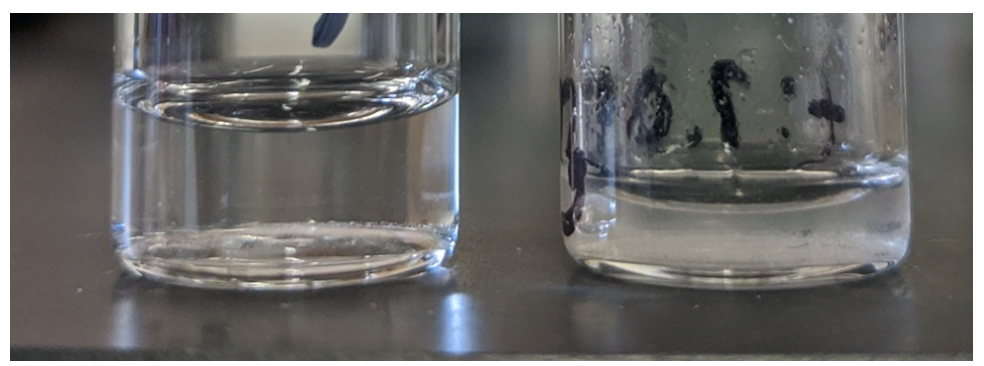

Figure S10: Polypeptoid solubility in water is demonstrated here for the $15 \mathrm{mer}$ at $200 \mu \mathrm{M}$ (well dispersed) and $1 \mathrm{mM}$ (cloudy). 


\section{S3. Experimental repeats}

The reported DEER $P\left(R_{e e}\right)$ uncertainty does not account for experimental error resulting from, for example, imperfect freezing or instrumental noise. However, two independent measurements of the $N=11$ polypeptoid agree within the uncertainty computed as described in section $\mathrm{S} 2$.

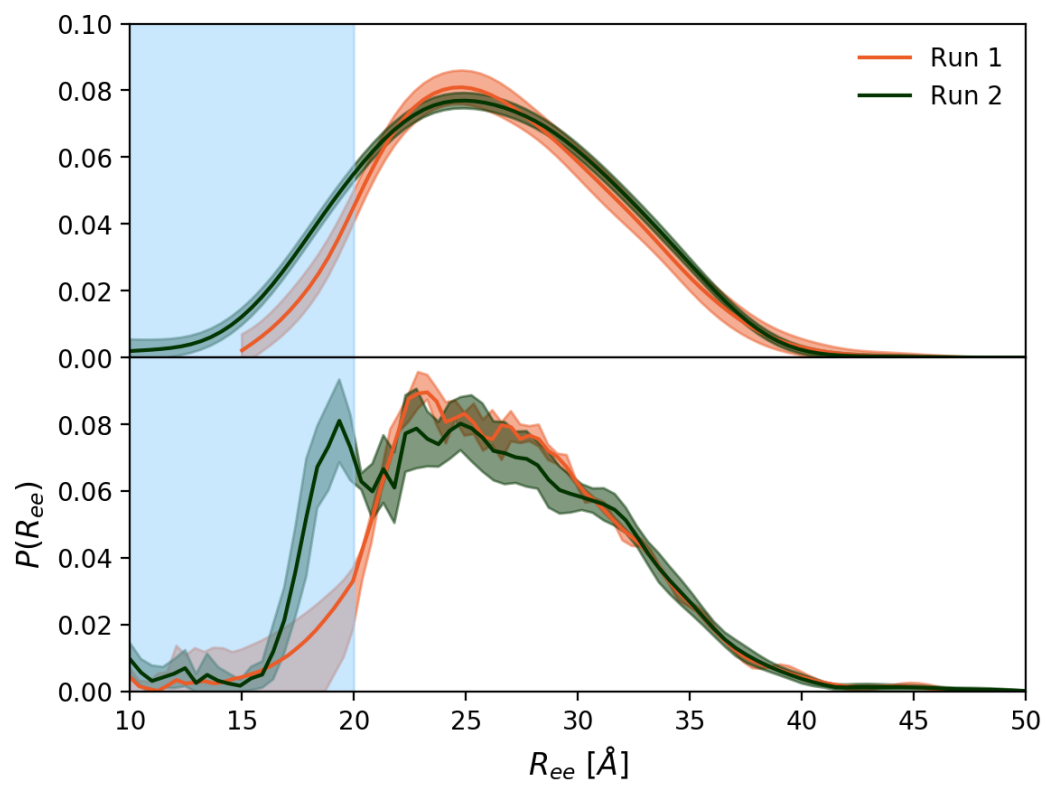

Figure S11: Fitted DEER distributions using LongDistances (top) and SF-SVD (bottom) from two DEER experiments on the $N=11$ polypeptoid. The second run is used in the main text (the first run contained some ESEEM in the timedomain). Uncertainties for the distribution for the SF-SVD fits are computed as described in section S2. 


\section{S4. Continuous-wave electron paramagnetic resonance (cw-EPR)}

Low-temperature cw-EPR spectra are acquired at $150 \mathrm{~K}$ of $200 \mu \mathrm{M}$ singly and doubly labeled 3mer polypeptoids. Samples are loaded into $3 \mathrm{~mm}$ ID quartz tubes and flash frozen in liquid nitrogen immediately prior to measurement. Spectra are acquired on a Bruker EMXplus Xband EPR spectrometer equipped with a Bruker ER4119HS-W1 resonator. Experimental parameters are: $0.63 \mathrm{~mW}$ microwave power, $15 \mathrm{~ms}$ conversion time, $10.24 \mathrm{~ms}$ time constant, and $0.1 \mathrm{mT}$ modulation amplitude. Scans are averaged until sufficiently high signal to noise is obtained ( $\sim 50-200$ scans).

The software package ShortDistances ${ }^{13}$ is used to extract a normal distribution of end-toend distances for a polypeptoid with 3 hydrophilic monomers. ShortDistances fits an average endto-end distance, $\left\langle R_{e e}\right\rangle$, and a full width half $\max (\mathrm{FWHM})$. In the main text, we report a standard deviation $(\sigma)$ rather than a FWHM using a conversion factor of $2 \sqrt{2 \ln (2)} \approx 2.355$. The calculated broadened spectra using the optimal average end to end distance, $\left\langle R_{e e}\right\rangle=16.5 \AA$, and $\sigma=2.2 \AA$ obtained from ShortDistances is compared to the raw data in Fig. S12.

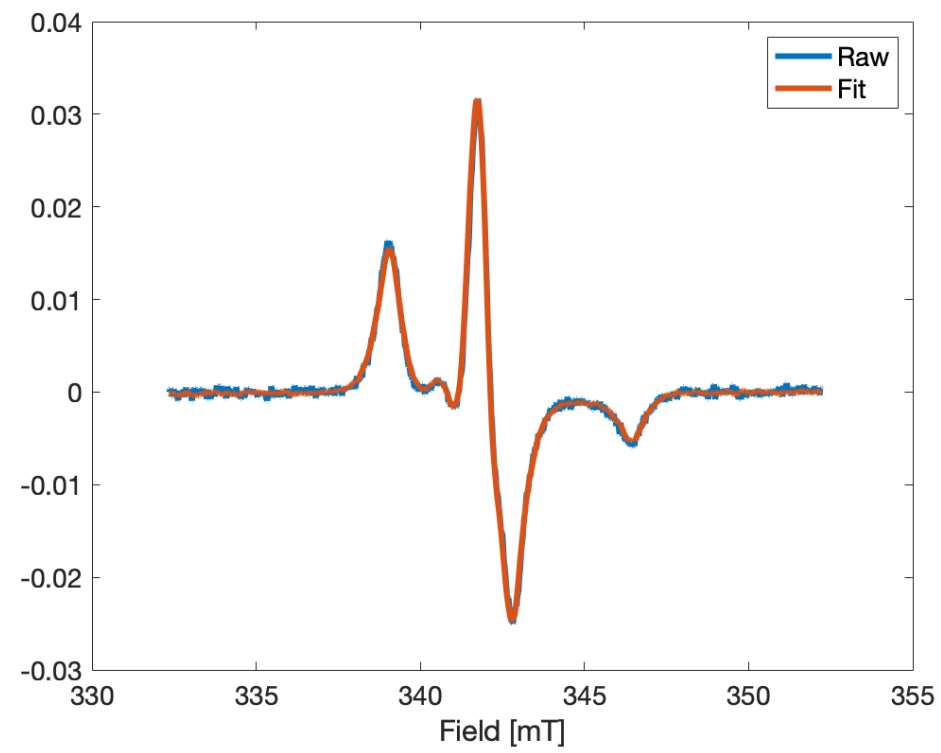

Figure S12. Cw-EPR spectra for the doubly labeled 3mer obtained from experiment (Raw) and calculated (Fit) by convolution of the singly-labeled experimental spectra with the contribution from dipolar broadening of the fitted $P\left(R_{e e}\right)$ distribution show good agreement.

We estimate uncertainties in the distance distribution obtained from cw-EPR by considering the impact of both $\left\langle R_{e e}\right\rangle$ and the FWHM on the resulting fit. We use the $\chi^{2}$ values obtained in ShortDistances to compare goodness of fit for different combinations of $\left\langle R_{e e}\right\rangle$ and the FWHM, with lower values of $\chi^{2}$ indicating a superior fit. To approximate the uncertainty in each parameter, we calculate the standard deviation from the covariance matrix of $\left\langle R_{e e}\right\rangle$ and FWHM. We first construct a Jacobian matrix with one column for each parameter (n columns) and one row for each data point (m rows) for which $\chi^{2}$ was calculated. Each element has the form $\frac{\partial \chi^{2}\left(y_{m}\right)}{\partial p_{n}}=$ $\frac{\left(\chi^{2}\left(p_{n}+d x_{n}\right)-\chi^{2}{ }_{\text {best }}\right)_{m}+\left(\chi^{2}\left(p_{n}-d x_{n}\right)-\chi^{2}{ }_{\text {best }}\right)_{m}}{2 d x_{n}}$ where $p_{n}$ indicates the parameter and $d x_{n}$ is the increment the parameter is varied by. The covariance matrix is then calculated using cov= 
$\frac{\chi^{2}{ }_{b e s t}}{m-n}\left(J_{n \times m}^{T} \times J_{m \times n}\right)^{-1}$. The diagonalized form of the covariance matrix contains standard deviations for each parameter along the diagonal and $95 \%$ confidence intervals are obtained by multiplying the standard deviation by 2 . This analysis yields confidence intervals of $\left\langle R_{e e}\right\rangle=$ $16.5 \pm 0.1 \AA$ and $\mathrm{FWHM}=5.2 \pm 2.8 \AA(\sigma=2.2 \pm 1.2 \AA)$. Fig. S13 shows $\chi^{2}$ while varying $\left\langle R_{e e}\right\rangle$ and holding the FWHM constant (Fig. S13a) and while holding $\left\langle R_{e e}\right\rangle$ but varying the FWHM (Fig. S13b). Clearly, $\chi^{2}$ is far more sensitive to changes in $\left\langle R_{e e}\right\rangle$ than the FWHM, as evidenced by the steeper increase in $\chi^{2}$ as $\left\langle R_{e e}\right\rangle$ is moved away from the optimum.
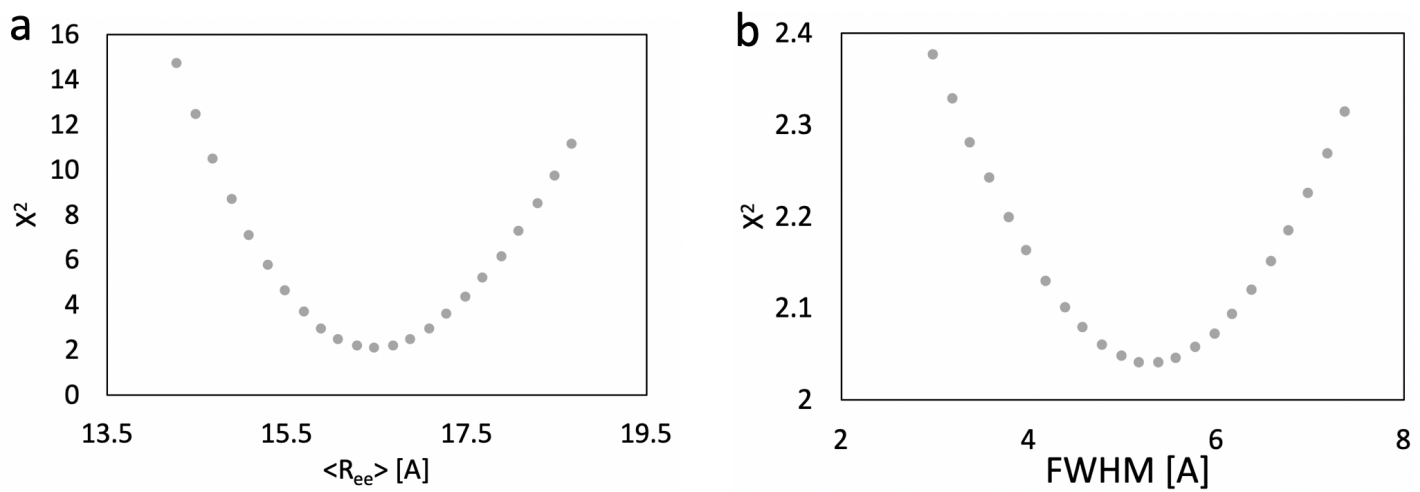

Figure S13: Dependence of goodness of fit on parameter selection. (a) $\chi^{2}$ shows a strong dependence on $\left\langle R_{e e}\right\rangle$ when the FWHM is held constant, in this case at the optimum value. (b) In contrast, $\chi^{2}$ varies far less with the FWHM. 
S5. Simulation box sizes (initial), number of water molecules, equilibration/production times, and number of independent, significant samples

\begin{tabular}{|c|c|c|c|c|c|c|c|}
\hline System & Forcefield & $\begin{array}{c}R_{e e, o o, \max } \\
[\AA]]\end{array}$ & $\boldsymbol{l}_{\boldsymbol{b}}[\AA]$ & $N_{w}$ & $\begin{array}{c}T_{\text {equil }} \\
\text { [ns] }\end{array}$ & $\begin{array}{c}T_{\text {prod }} \\
{[\mathrm{ns}]}\end{array}$ & $N_{s}$ \\
\hline$N=1$ & MFTOID & 9.2 & 36.3752 & 1490 & 400 & 1,400 & 737 \\
\hline$N=1$ & Modified CGenFF & 9.2 & 36.3752 & 1490 & 200 & 2,000 & 990 \\
\hline$N=2$ & MFTOID & 15.9 & 39.8024 & 2091 & 400 & 2,000 & 417 \\
\hline$N=2$ & Modified CGenFF & 15.9 & 39.8024 & 2091 & 420 & 2,400 & 628 \\
\hline$N=3$ & MFTOID & 16.9 & 44.3112 & 2809 & 200 & 4,000 & 263 \\
\hline$N=3$ & Modified CGenFF & 16.9 & 44.3112 & 2809 & 420 & 3,600 & 600 \\
\hline$N=5$ & MFTOID & 23.1 & 51.0089 & 4284 & 200 & 2,800 & 101 \\
\hline$N=5$ & Modified CGenFF & 23.1 & 51.0089 & 4284 & 220 & 3,600 & 227 \\
\hline$N=7$ & MFTOID & 30.3 & 58.3857 & 6552 & 600 & 4,000 & 198 \\
\hline$N=7$ & Modified CGenFF & 30.3 & 58.3857 & 6552 & 220 & 4,800 & 369 \\
\hline$N=9$ & MFTOID & 37.5 & 65.2181 & 8908 & 600 & 4,000 & 253 \\
\hline$N=9$ & Modified CGenFF & 37.5 & 65.2181 & 8908 & 220 & 11,000 & 429 \\
\hline$N=11$ & MFTOID & 45.4 & 72.6911 & 12341 & 600 & 6,000 & 180 \\
\hline$N=11$ & $\begin{array}{l}\text { MFTOID with implicit } \\
\text { solvent }\end{array}$ & 45.4 & 72.6911 & N/A & 600 & 4,000 & 666 \\
\hline$N=11$ & MFTOID with SPC/E & 45.4 & 72.6911 & 12341 & 1,400 & 8,800 & 142 \\
\hline$N=11$ & MFTOID with OPC4 & 45.4 & 72.6911 & 12576 & 600 & 7,600 & 106 \\
\hline $\begin{array}{l}N=11 \\
\text { (unlabeled) }\end{array}$ & MFTOID & $\mathrm{N} / \mathrm{A}$ & 64.2859 & 8644 & 400 & 2,000 & 152 \\
\hline$N=11$ & Modified AMBER & 45.4 & 72.6911 & 12341 & 600 & 10,000 & 116 \\
\hline$N=11$ & $\begin{array}{l}\text { Modified AMBER with } \\
\text { implicit solvent }\end{array}$ & 45.4 & 72.6911 & N/A & 600 & 6,000 & 750 \\
\hline$N=11$ & GAFF $-\varphi$ & 45.4 & 72.6911 & 12341 & 1,400 & 8,000 & 95 \\
\hline$N=11$ & $\begin{array}{l}\text { GAFF- } \varphi \text { with implicit } \\
\text { solvent }\end{array}$ & 45.4 & 72.6911 & $\mathrm{~N} / \mathrm{A}$ & 1,000 & 6,000 & 1764 \\
\hline$N=11$ & Modified CGenFF & 45.4 & 72.6911 & 12341 & 300 & 5,200 & 158 \\
\hline$N=11$ & $\begin{array}{l}\text { Modified CGenFF with } \\
\text { implicit solvent }\end{array}$ & 45.4 & 72.6911 & $\mathrm{~N} / \mathrm{A}$ & 600 & 4,000 & 625 \\
\hline$N=13$ & MFTOID & 53.0 & 81.7029 & 17754 & 600 & 6,200 & 134 \\
\hline$N=13$ & Modified CGenFF & 53.0 & 81.7029 & 17754 & 300 & 7,600 & 104 \\
\hline$N=15$ & MFTOID & 62.5 & 89.4396 & 23293 & 600 & 6,600 & 158 \\
\hline$N=15$ & Modified CGenFF & 62.5 & 89.4396 & 23293 & 820 & 6,400 & 46 \\
\hline$N=19$ & MFTOID & 80.0 & 106.0111 & 38639 & 500 & 5,600 & 114 \\
\hline$N=19$ & Modified CGenFF & 80.0 & 106.0111 & 38639 & 720 & 3,000 & 30 \\
\hline Disarcosine & MFTOID & N/A & 40. & 2156 & 200 & 5,000 & 5,000 \\
\hline
\end{tabular}

Table S2: Besides disarcosine, all systems correspond to varying lengths of the hydrophilic sequence shown in Fig. 1 of the main text. $l_{b}$ is the initial box length and $N_{w}$ is the number of water molecules, set as described in the main text. $T_{\text {equil }}$ and $T_{\text {prod }}$ give the lengths of the equilibration (varying weights) and production (fixed weights) simulations, respectively. $N_{S}$ is an estimate of the number of significant and independent samples, computed as described in section $\mathrm{S} 13$. The maximum end-to-end distance between spin label oxygen radicals, $R_{e e, o o, \max }$, is estimated from the initial, fully extended configuration, which is created in Avogadro 1.2.0 and then geometry minimized. ${ }^{14}$ All forcefields are simulated with the water model specified in section S8 unless otherwise stated. 


\section{S6. Partial charge assignment}

For the partial charges computed using ESP calculations (hydrophilic monomers for the modified AMBER forcefield and spin-label monomers for the modified AMBER and GAFF- $\phi$ forcefields), we create the structures in Avogadro 1.2.0 $0^{14}$ and perform a geometry minimization. Then, we perform a geometry optimization and then a single-point calculation in Gaussian $16^{15}$ using the default SCF procedure and outputting charges derived from the ESP to an Antechamber file using 8 points per unit area and 7 layers. The spin multiplicity is set to 1 for homopolymers of the hydrophilic monomers (a spin multiplicity of 3 results in higher energies) and 2 for calculations with the spin label (a spin multiplicity of 4 results in higher energies).

To obtain charges for the hydrophilic monomers, we then use RESP fitting to fit partial charges on 8-mer homopolymers of the hydrophilic monomers. Then, we use the prepgen tool in AmberTools $16^{16}$ to adjust the charges so that each monomer is net neutral. We average the charges for all monomers except the N-terminal monomer. This procedure is different from the charging procedure for the modified AMBER forcefield in Ref. [17], which involves performing ESP and RESP calculations on an entire polypeptoid and is intractable for longer polypeptoids. We repeat this calculation varying the length of the homopolymer and find that the average charges converge by 8 monomers (Fig. S15 and S16). We also repeat the calculation for shorter homopolymers using the B3LYP functional with the $6-311++\mathrm{G}(\mathrm{d}, \mathrm{p})$ basis set and find convergence to similar values (Fig. S15 and S16). Charges for the C-terminal amino group are the charges fit for the group in the homopolymer of 8 NME monomers.

For the N-terminal spin-label monomer, charges for a twomer with the spin-label monomer at the N-terminus and the NME monomer at the C-terminus are derived from an ESP calculation using the procedure above with the B3LYP functional and the $6-311++G(d, p)$ basis set and then fit with RESP. Then, prepgen is used to adjust charges so that each monomer is net neutral. The same procedure is followed for the C-terminal spin-label monomer, except with a twomer with the NME monomer at the N-terminus and the spin-label monomer at the C-terminus.

For the hydrophilic monomers for the GAFF- $\phi$ forcefield, we create twomers with an Nterminal alcohol monomer and the monomer of interest at the C-terminus in Avogadro and perform a geometry minimization. Partial charges for the twomer are assigned with the AM1-BCC model using AmberTools16. Then, we use the prepgen tool in AmberTools16 to adjust the charges so that each monomer is net neutral. Charges for the C-terminal amino group are fit by fitting charges for a twomer of the alcohol monomer, after which charges are adjusted for the amino group so that it is net neutral.

The MFTOID forcefield and the modified CGenFF forcefield share the same partial charges. The charge on the backbone nitrogens are set to -0.42 , the value fit in Mirijanian et al.. ${ }^{18}$ For the alcohol monomer, charges for the alcohol group, adjacent methylene group, and backbone carbonyl group are set to the same as those for serine in the CHARMM22 forcefield. Charges for the next methylene group (CG in Fig. S14) are set to charges for the methylene group in the same position in proline in the CHARMM22 forcefield. Charges for the remaining hydrogens are set to 0.09 , to be consistent with charges on other methylene hydrogens in the amino acids for the CHARMM22 forcefield, and the remaining charge is divided evenly between the backbone methylene and first sidechain methylene (CA and CB, respectively, in Fig. S14) to make the monomer net neutral. For the NME monomer, the backbone charges and first sidechain methylene (CB in Fig. S14) charges are set to the same as for the alcohol monomer. The ether group charges (CG, OG, CD and associated hydrogens in Fig. S14) are set to those for linear ether groups defined in Ref. [19]. For the spin label monomer, the backbone charges are set to the same as for the alcohol 
monomer. Charges for the ring atoms, excluding the one bonded to the backbone nitrogen (CB in Fig. S14), are the same as in Ref. [20]. Charges for all hydrogens are 0.09, as in Ref. [20]. The net charge in the sidechain ring, excluding $\mathrm{CB}$ and its hydrogen (HB1 in Fig. S14), are divided evenly among the methyl groups, giving a charge of -0.3305 . The net charge for the backbone $\mathrm{CB}$ is assigned to $\mathrm{CB}$, giving a charge of 0.12 . For the N-terminal monomers, the charge for the Nterminal hydrogen is set to 0.33 , to be consistent with the charge for an N-terminal hydrogen for peptides in CHARMM22 (defined for NH3+ terminal groups). The charge on the backbone nitrogen is reduced to -0.75 to keep the monomer net neutral. For the C-terminal amino group, the charges on the hydrogen are set to 0.33 , the same as the N-terminal hydrogen, and the charge on the nitrogen is set to 0.66 to make the group net neutral.

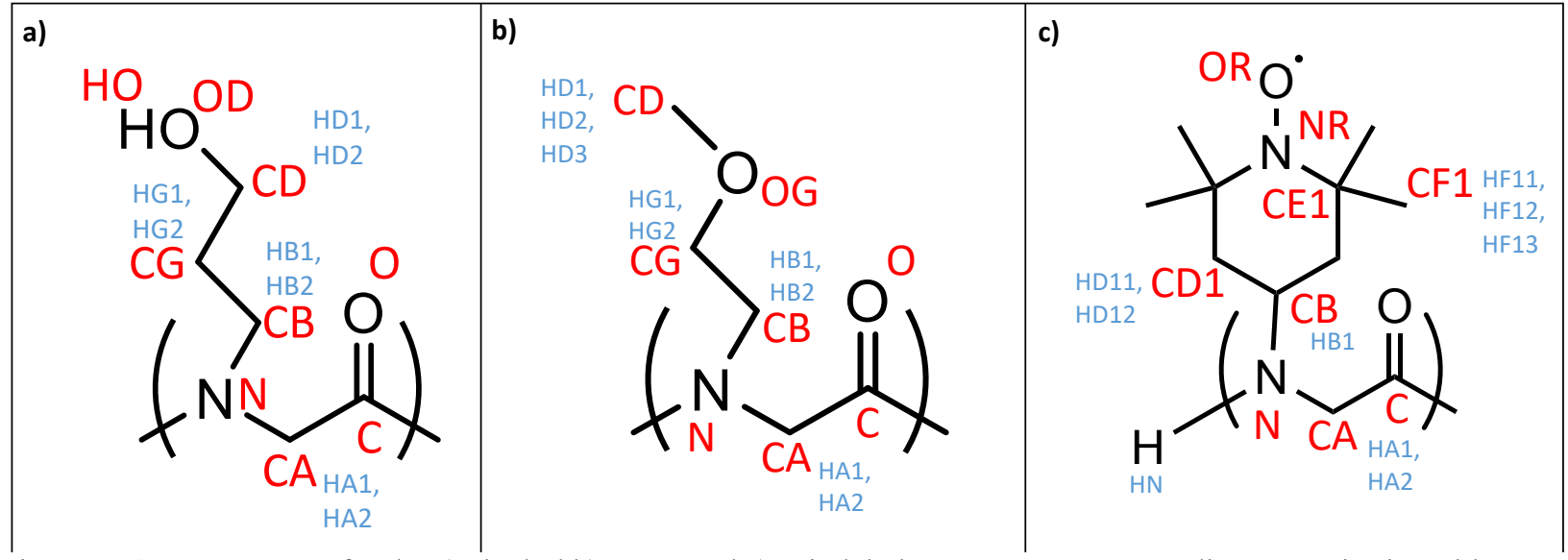

Figure S14: Atom names for the a) alcohol b) NME and c) spin label monomer, corresponding to entries in Tables S3S7.

\begin{tabular}{|c|c|c|c|}
\hline Atom & Modified AMBER & MFTOID / modified CGenFF & GAFF- $\varphi$ \\
\hline $\mathrm{C}$ & 0.760 & 0.51 & 0.641 \\
\hline $\mathrm{O}$ & -0.597 & -0.51 & -0.605 \\
\hline $\mathrm{CA}$ & -0.294 & 0.03 & 0.018 \\
\hline $\mathrm{HA}^{*}$ & 0.143 & 0.09 & 0.090 \\
\hline $\mathrm{N}$ & -0.233 & -0.42 & -0.469 \\
\hline $\mathrm{CB}$ & -0.174 & 0.03 & 0.094 \\
\hline $\mathrm{HB}^{*}$ & 0.073 & 0.09 & 0.061 \\
\hline $\mathrm{CG}$ & 0.067 & -0.18 & -0.144 \\
\hline $\mathrm{HG}^{*}$ & 0.018 & 0.09 & 0.059 \\
\hline $\mathrm{CD}$ & 0.316 & 0.05 & 0.131 \\
\hline $\mathrm{HD}^{*}$ & -0.010 & 0.09 & 0.047 \\
\hline OD & -0.712 & -0.66 & -0.583 \\
\hline $\mathrm{HO}$ & 0.420 & 0.43 & 0.403 \\
\hline
\end{tabular}

Table S3: Partial charges for atoms on the alcohol monomer used with each polypeptoid forcefield. Atom names are shown in Fig. S14. Asterisks signal that there are multiple atoms with the same charge (e.g. HA1 and HA2).

\begin{tabular}{|c|c|c|c|}
\hline Atom & Modified AMBER & MFTOID / modified CGenFF & GAFF- $\boldsymbol{\varphi}$ \\
\hline C & 0.759 & 0.51 & 0.644 \\
\hline O & -0.629 & -0.51 & -0.605 \\
\hline CA & -0.344 & 0.03 & 0.017 \\
\hline HA $^{*}$ & 0.160 & 0.09 & 0.091 \\
\hline N & -0.235 & -0.42 & -0.466 \\
\hline CB & -0.069 & 0.03 & 0.087 \\
\hline
\end{tabular}




\begin{tabular}{|c|c|c|c|}
\hline $\mathrm{HB}^{*}$ & 0.071 & 0.09 & 0.073 \\
\hline $\mathrm{CG}^{*}$ & 0.206 & -0.01 & 0.106 \\
\hline $\mathrm{HG}^{*}$ & 0.043 & 0.09 & 0.038 \\
\hline $\mathrm{OG}$ & -0.429 & -0.34 & -0.417 \\
\hline $\mathrm{CD}$ & 0.039 & -0.1 & 0.120 \\
\hline $\mathrm{HD}^{*}$ & 0.051 & 0.09 & 0.036 \\
\hline
\end{tabular}

Table S4: Same as Table S3 but for the NME monomer.

\begin{tabular}{|c|c|c|}
\hline Atom & Modified AMBER / GAFF- $\boldsymbol{\varphi}$ & MFTOID / modified CGenFF \\
\hline $\mathrm{C}$ & 0.820 & 0.51 \\
\hline $\mathrm{O}$ & -0.635 & -0.51 \\
\hline $\mathrm{CA}$ & -0.351 & 0.03 \\
\hline $\mathrm{HA}^{*}$ & 0.130 & 0.09 \\
\hline $\mathrm{N}$ & 0.004 & -0.42 \\
\hline $\mathrm{CB}$ & 0.015 & 0.12 \\
\hline $\mathrm{HB} 1$ & 0.105 & 0.09 \\
\hline $\mathrm{CD}^{*}$ & -0.484 & -0.237 \\
\hline $\mathrm{HD}^{*}$ & 0.106 & 0.09 \\
\hline $\mathrm{CE}^{*}$ & 0.633 & 0.284 \\
\hline $\mathrm{CF}^{*}$ & -0.330 & -0.3305 \\
\hline HF* & 0.073 & 0.09 \\
\hline NR & -0.079 & 0.167 \\
\hline OR & -0.413 & -0.379 \\
\hline
\end{tabular}

Table S5: Same as Table S3 but for the C-terminal spin-label.

\begin{tabular}{|c|c|c|}
\hline Atom & Modified AMBER / GAFF- $\boldsymbol{\varphi}$ & MFTOID / modified CGenFF \\
\hline $\mathrm{C}$ & 0.698 & 0.51 \\
\hline $\mathrm{O}$ & -0.565 & -0.51 \\
\hline $\mathrm{CA}$ & -0.094 & 0.03 \\
\hline HA & 0.082 & 0.09 \\
\hline $\mathrm{N}$ & 0.049 & -0.75 \\
\hline $\mathrm{HN}$ & 0.334 & 0.33 \\
\hline $\mathrm{CB}$ & 0.361 & 0.12 \\
\hline $\mathrm{HB} 1$ & 0.033 & 0.09 \\
\hline $\mathrm{CD}^{*}$ & -0.672 & -0.237 \\
\hline $\mathrm{HD}^{*}$ & 0.181 & 0.09 \\
\hline $\mathrm{CE}^{*}$ & 0.557 & 0.284 \\
\hline $\mathrm{CF}^{*}$ & -0.257 & -0.3305 \\
\hline $\mathrm{HF}^{*}$ & 0.056 & 0.09 \\
\hline NR & 0.049 & 0.167 \\
\hline OR & -0.432 & -0.379 \\
\hline
\end{tabular}

Table S6: Same as Table S3 but for the N-terminal spin-label.

\begin{tabular}{|c|c|c|c|}
\hline Atom & Modified AMBER & MFTOID / modified CGenFF & GAFF- $\boldsymbol{\varphi}$ \\
\hline $\mathrm{N}$ & -0.974 & -0.66 & -0.644 \\
\hline $\mathrm{H}^{*}$ & 0.487 & 0.33 & 0.321 \\
\hline
\end{tabular}

Table S7: Same as Table S3 but for the C-terminal amino group. 


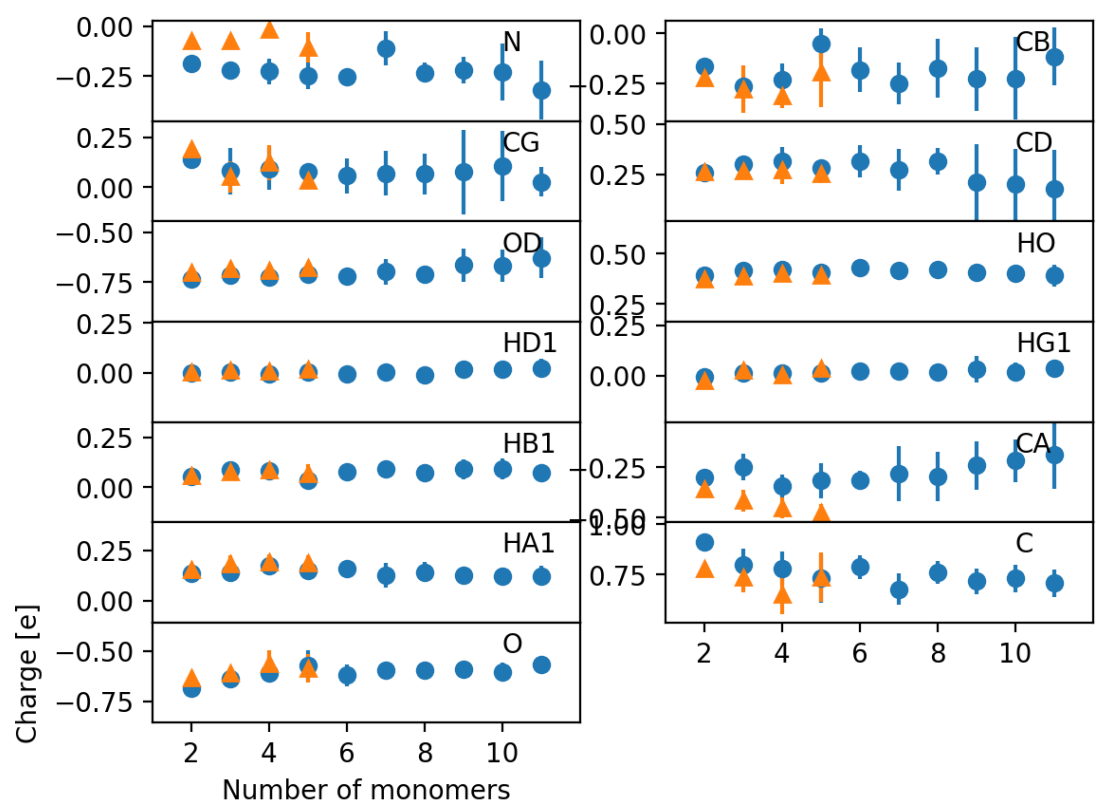

Figure S15: Each plot shows the average partial charge on an atom (names correspond to those in Fig. S14) in homopolymers of the alcohol monomer of varying length. The blue circles correspond to calculations using HartreeFock level of theory with the 6-31G(d) basis set, while the orange triangles correspond to calculations using the B3LYP functional with the 6-311++G(d,p) basis set. The error bars show the standard deviation in the partial charge over the homopolymer. As the number of monomers increases, the average converges, but the longer homopolymers have access to more conformations, therefore increasing the standard deviation. Here, we use the partial charges computed for a homopolymer with 8 monomers. The y-axis for each plot is $0.5 \mathrm{e}$ long. The $\mathrm{x}$-axis is the total number of monomers in the homopolymer, but averages are taken excluding the $\mathrm{N}$-terminal monomer.

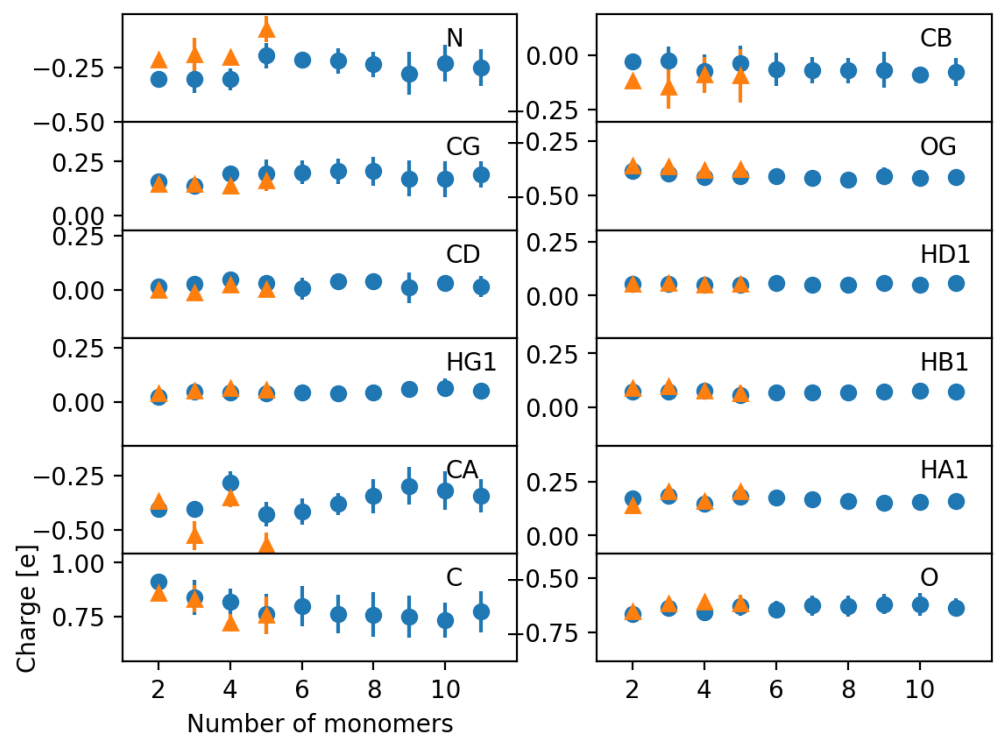

Figure S16: The same as Fig. S15, but for the NME monomer. 


\section{S7. Atom type assignment}

For the modified AMBER and GAFF- $\varphi$ forcefields, we perform atom type assignment to types in the AMBER99SB-ILDN and GAFF forcefields, respectively, using AmberTools 16. ${ }^{16}$ Stendardo et al. ${ }^{21}$ provides atom types for the spin labels for the AMBER forcefield. For the MFTOID ${ }^{18}$ forcefield, we perform atom type assignment to types in the CHARMM22 ${ }^{22}$ and CGenFF3.0.1 ${ }^{23}$ (downloaded 12/10/2018) forcefields (and one new type, TC, from MFTOID ${ }^{18}$ ) using Mirijanian et al., ${ }^{18}$ analogous amino acids, ParamChem, ${ }^{24,25}$ and Sezer et al. ${ }^{20}$ for the spin label. For the modified CGenFF forcefield, we perform atom type assignment to types in the CGenFF4.1 (charmm36-mar2019.ff.tgz downloaded from http://mackerell.umaryland.edu/charmm_ff.shtml\#gromacs on 10/24/2019) or CHARMM22 forcefields (and one new type, NTOID, from Weiser et al. ${ }^{26}$ ) using ParamChem, ${ }^{24,25}$ Weiser et al. ${ }^{26}$ and Sezer et al. ${ }^{20}$ for the spin label.

\begin{tabular}{|c|c|c|c|c|}
\hline Atom & Modified AMBER & GAFF- $\varphi$ & MFTOID & Modified CGenFF \\
\hline $\mathrm{C}$ & $\begin{array}{l}\text { C (AMBER99SB- } \\
\text { ILDN) }\end{array}$ & $\mathrm{c}(\mathrm{GAFF}$ v1.8) & TC (MFTOID) & $\begin{array}{l}\text { CG2O1 (CGenFF type, } \\
\text { assigned from ParamChem) }\end{array}$ \\
\hline $\mathrm{O}$ & $\begin{array}{l}\text { O (AMBER99SB- } \\
\text { ILDN) }\end{array}$ & o (GAFF v1.8) & $\begin{array}{l}\text { O (CHARMM22 type, assigned as } \\
\text { in disarcosine in Mirijanian et al.) }\end{array}$ & $\begin{array}{l}\text { OG2D1 (CGenFF type, } \\
\text { assigned from ParamChem) }\end{array}$ \\
\hline CA & $\begin{array}{l}\text { CT (AMBER99SB- } \\
\text { ILDN) }\end{array}$ & c3 (GAFF v1.8) & $\begin{array}{l}\text { CT2 (CHARMM22 type, assigned } \\
\text { as in disarcosine in Mirijanian et al.) }\end{array}$ & $\begin{array}{l}\text { CG321 (CGenFF type, } \\
\text { assigned from ParamChem) }\end{array}$ \\
\hline $\mathrm{HA}^{*}$ & $\begin{array}{l}\text { H1 (AMBER99SB- } \\
\text { ILDN) }\end{array}$ & h1 (GAFF v1.8) & $\begin{array}{l}\text { HB (CHARMM22 type, assigned as } \\
\text { in disarcosine in Mirijanian et al.) }\end{array}$ & $\begin{array}{c}\text { HGA2 (CGenFF type, } \\
\text { assigned from ParamChem) }\end{array}$ \\
\hline $\mathrm{N}$ & $\begin{array}{l}\text { NT (AMBER99SB- } \\
\text { ILDN) }\end{array}$ & n (GAFF v1.8) & $\begin{array}{l}\text { NH1 (CHARMM22 type, assigned } \\
\text { as in disarcosine in Mirijanian et al.) }\end{array}$ & NTOID (Weiser et al.) \\
\hline $\mathrm{CB}$ & $\begin{array}{l}\text { CT (AMBER99SB- } \\
\text { ILDN) }\end{array}$ & c3 (GAFF v1.8) & $\begin{array}{l}\text { CT2 (CHARMM22 type, assigned } \\
\text { as in disarcosine in Mirijanian et al.) }\end{array}$ & $\begin{array}{c}\text { CG321 (CGenFF type, } \\
\text { assigned from ParamChem) }\end{array}$ \\
\hline $\mathrm{HB}^{*}$ & $\begin{array}{l}\text { H1 (AMBER99SB- } \\
\text { ILDN) }\end{array}$ & h1 (GAFF v1.8) & $\begin{array}{l}\text { HA (CHARMM22 type, assigned as } \\
\text { in disarcosine in Mirijanian et al.) }\end{array}$ & $\begin{array}{l}\text { HGA2 (CGenFF type, } \\
\text { assigned from ParamChem) }\end{array}$ \\
\hline CG & $\begin{array}{l}\text { CT (AMBER99SB- } \\
\text { ILDN) }\end{array}$ & c3 (GAFF v1.8) & CG2 (CHARMM22 SER CB) & $\begin{array}{l}\text { CG321 (CGenFF type, } \\
\text { assigned from ParamChem) }\end{array}$ \\
\hline $\mathrm{HG}^{*}$ & $\begin{array}{l}\text { HC (AMBER99SB- } \\
\text { ILDN) }\end{array}$ & hc (GAFF v1.8) & HA (CHARMM22 SER HB*) & $\begin{array}{c}\text { HGA2 (CGenFF type, } \\
\text { assigned from ParamChem) }\end{array}$ \\
\hline $\mathrm{CD}$ & $\begin{array}{l}\text { CT (AMBER99SB- } \\
\text { ILDN) }\end{array}$ & c3 (GAFF v1.8) & CT2 (CHARMM22 SER CB) & $\begin{array}{c}\text { CG321 (CGenFF type, } \\
\text { assigned from ParamChem) }\end{array}$ \\
\hline $\mathrm{HD}^{*}$ & $\begin{array}{l}\text { H1 (AMBER99SB- } \\
\text { ILDN) }\end{array}$ & h1 (GAFF v1.8) & HA (CHARMM22 SER HB*) & $\begin{array}{l}\text { HGA2 (CGenFF type, } \\
\text { assigned from ParamChem) }\end{array}$ \\
\hline OD & $\begin{array}{l}\text { OH (AMBER99SB- } \\
\text { ILDN) }\end{array}$ & oh (GAFF v1.8) & OH1 (CHARMM22 SER OG) & $\begin{array}{l}\text { OG311 (CGenFF type, } \\
\text { assigned from ParamChem) }\end{array}$ \\
\hline $\mathrm{HO}$ & $\begin{array}{l}\text { HO (AMBER99SB- } \\
\text { ILDN) }\end{array}$ & ho (GAFF v1.8) & H (CHARMM22 SER HG1) & $\begin{array}{c}\text { HGP1 (CGenFF type, } \\
\text { assigned from ParamChem) }\end{array}$ \\
\hline
\end{tabular}

Table S8: Atom types for atoms on the alcohol monomer used with each polypeptoid forcefield. Atom names are shown in Fig. S14. Asterisks signal that there are multiple atoms with the same assignment (e.g. HA1 and HA2).

\begin{tabular}{|c|c|c|c|c|}
\hline Atom & Modified AMBER & GAFF- $\varphi$ & MFTOID & Modified CGenFF \\
\hline $\mathrm{C}$ & $\begin{array}{c}\text { C (AMBER99SB- } \\
\text { ILDN) }\end{array}$ & $\mathrm{c}$ (GAFF v1.8) & TC (MFTOID) & $\begin{array}{c}\text { CG2O1 (CGenFF type, } \\
\text { assigned from ParamChem) }\end{array}$ \\
\hline $\mathrm{O}$ & $\begin{array}{c}\text { O (AMBER99SB- } \\
\text { ILDN) }\end{array}$ & $\mathrm{o}(\mathrm{GAFF}$ v1.8) & $\begin{array}{c}\text { O (CHARMM22 type, assigned as in } \\
\text { disarcosine in Mirijanian et al.) }\end{array}$ & $\begin{array}{c}\text { OG2D1 (CGenFF type, } \\
\text { assigned from ParamChem) }\end{array}$ \\
\hline CA & $\begin{array}{c}\text { CT (AMBER99SB- } \\
\text { ILDN) }\end{array}$ & $\mathrm{c} 3$ (GAFF v1.8) & $\begin{array}{c}\text { CT2 (CHARMM22 type, assigned as } \\
\text { in disarcosine in Mirijanian et al.) }\end{array}$ & $\begin{array}{c}\text { CG321 (CGenFF type, } \\
\text { assigned from ParamChem) }\end{array}$ \\
\hline HA $*$ & $\begin{array}{c}\text { H1 (AMBER99SB- } \\
\text { ILDN) }\end{array}$ & h1 (GAFF v1.8) & $\begin{array}{c}\text { HB (CHARMM22 type, assigned as } \\
\text { in disarcosine in Mirijanian et al.) }\end{array}$ & $\begin{array}{c}\text { HGA2 (CGenFF type, } \\
\text { assigned from ParamChem) }\end{array}$ \\
\hline
\end{tabular}




\begin{tabular}{|c|c|c|c|c|}
\hline $\mathrm{N}$ & $\begin{array}{l}\text { N (AMBER99SB- } \\
\text { ILDN) }\end{array}$ & $\mathrm{n}(\mathrm{GAFF}$ v1.8) & $\begin{array}{l}\text { NH1 (CHARMM22 type, assigned as } \\
\text { in disarcosine in Mirijanian et al.) }\end{array}$ & NTOID (Weiser et al.) \\
\hline $\mathrm{CB}$ & $\begin{array}{l}\text { CT (AMBER99SB- } \\
\text { ILDN) }\end{array}$ & c3 (GAFF v1.8) & $\begin{array}{l}\text { CT2 (CHARMM22 type, assigned as } \\
\text { in disarcosine in Mirijanian et al.) }\end{array}$ & $\begin{array}{l}\text { CG321 (CGenFF type, } \\
\text { assigned from ParamChem) }\end{array}$ \\
\hline $\mathrm{HB}^{*}$ & $\begin{array}{l}\text { H1 (AMBER99SB- } \\
\text { ILDN) }\end{array}$ & h1 (GAFF v1.8) & $\begin{array}{l}\text { HA (CHARMM22 type, assigned as } \\
\text { in disarcosine in Mirijanian et al.) }\end{array}$ & $\begin{array}{c}\text { HGA2 (CGenFF type, } \\
\text { assigned from ParamChem) }\end{array}$ \\
\hline $\mathrm{CG}$ & $\begin{array}{l}\text { CT (AMBER99SB- } \\
\text { ILDN) }\end{array}$ & c3 (GAFF v1.8) & $\begin{array}{c}\text { CT2 (CHARMM22 type, assigned to } \\
\text { be consistent with other sidechain } \\
\text { CH2 groups) }\end{array}$ & $\begin{array}{l}\text { CG321 (CGenFF type, } \\
\text { assigned from ParamChem) }\end{array}$ \\
\hline $\mathrm{HG}^{*}$ & $\begin{array}{l}\text { H1 (AMBER99SB- } \\
\text { ILDN) }\end{array}$ & h1 (GAFF v1.8) & $\begin{array}{c}\text { HA (CHARMM22 type, assigned to } \\
\text { be consistent with other sidechain } \\
\text { CH2 groups) }\end{array}$ & $\begin{array}{l}\text { HGA2 (CGenFF type, } \\
\text { assigned from ParamChem) }\end{array}$ \\
\hline $\mathrm{OG}$ & $\begin{array}{l}\text { OS (AMBER99SB- } \\
\text { ILDN) }\end{array}$ & os (GAFF v1.8) & $\begin{array}{l}\text { OG301 (CGenFF type, assigned from } \\
\text { ParamChem) }\end{array}$ & $\begin{array}{l}\text { OG301 (CGenFF type, } \\
\text { assigned from ParamChem) }\end{array}$ \\
\hline $\mathrm{CD}$ & $\begin{array}{l}\text { CT (AMBER99SB- } \\
\text { ILDN) }\end{array}$ & c3 (GAFF v1.8) & $\begin{array}{c}\text { CT2 (CHARMM22 type, assigned to } \\
\text { be consistent with other sidechain } \\
\text { CH2 groups) }\end{array}$ & $\begin{array}{l}\text { CG331 (CGenFF type, } \\
\text { assigned from ParamChem) }\end{array}$ \\
\hline $\mathrm{HD}^{*}$ & $\begin{array}{l}\text { H1 (AMBER99SB- } \\
\text { ILDN) }\end{array}$ & h1 (GAFF v1.8) & $\begin{array}{l}\text { HA (CHARMM22 type, assigned to } \\
\text { be consistent with other sidechain } \\
\text { CH2 groups) }\end{array}$ & $\begin{array}{c}\text { HGA3 (CGenFF type, } \\
\text { assigned from ParamChem) }\end{array}$ \\
\hline
\end{tabular}

Table S9: Same as Table S8 but for the NME monomer.

\begin{tabular}{|c|c|c|c|c|}
\hline Atom & Modified AMBER & GAFF- $\varphi$ & MFTOID & Modified CGenFF \\
\hline $\mathrm{N}$ & $\begin{array}{c}\text { N (AMBER99SB- } \\
\text { ILDN) }\end{array}$ & n (GAFF v1.8) & $\begin{array}{c}\text { NH1 (CHARMM22 type, assigned to } \\
\text { be consistent with other peptoid } \\
\text { nitrogens) }\end{array}$ & $\begin{array}{c}\text { NTOID (Weiser et al., } \\
\text { assigned to be consistent with } \\
\text { other peptoid nitrogens) }\end{array}$ \\
\hline $\mathrm{H}^{*}$ & $\begin{array}{c}\mathrm{H} \text { (AMBER99SB- } \\
\text { ILDN) }\end{array}$ & hn (GAFF v1.8) & $\begin{array}{c}\text { HC (CHARMM22 type for hydrogen } \\
\text { in N terminal group) }\end{array}$ & $\begin{array}{c}\text { HGP1 (CGenFF type, } \\
\text { assigned from ParamChem) }\end{array}$ \\
\hline
\end{tabular}

Table S10: Same as Table S8 but for the C-terminal amino group.

\begin{tabular}{|c|c|c|c|c|}
\hline Atom & Modified AMBER & GAFF- $\phi$ & MFTOID & Modified CGenFF \\
\hline $\mathrm{C}$ & $\begin{array}{l}\text { C (AMBER99SB- } \\
\text { ILDN) }\end{array}$ & c (GAFF v1.8) & TC (MFTOID) & $\begin{array}{c}\text { CG2O1 (CGenFF type, } \\
\text { assigned from ParamChem) }\end{array}$ \\
\hline $\mathrm{O}$ & $\begin{array}{l}\text { O (AMBER99SB- } \\
\text { ILDN) }\end{array}$ & o (GAFF v1.8) & $\begin{array}{l}\text { O (CHARMM22 type, assigned as in } \\
\text { disarcosine in Mirijanian et al.) }\end{array}$ & $\begin{array}{l}\text { OG2D1 (CGenFF type, } \\
\text { assigned from ParamChem) }\end{array}$ \\
\hline CA & $\begin{array}{l}\text { CT (AMBER99SB- } \\
\text { ILDN) }\end{array}$ & c3 (GAFF v1.8) & $\begin{array}{l}\text { CT2 (CHARMM22 type, assigned as } \\
\text { in disarcosine in Mirijanian et al.) }\end{array}$ & $\begin{array}{l}\text { CG321 (CGenFF type, } \\
\text { assigned from ParamChem) }\end{array}$ \\
\hline $\mathrm{HA}^{*}$ & $\begin{array}{l}\text { H1 (AMBER99SB- } \\
\text { ILDN) }\end{array}$ & h1 (GAFF v1.8) & $\begin{array}{l}\text { HB (CHARMM22 type, assigned as } \\
\text { in disarcosine in Mirijanian et al.) }\end{array}$ & $\begin{array}{l}\text { HGA2 (CGenFF type, } \\
\text { assigned from ParamChem) }\end{array}$ \\
\hline $\mathrm{N}$ & $\begin{array}{c}\text { NT (N-terminal), N } \\
\text { (otherwise) } \\
\text { (AMBER99SB- } \\
\text { ILDN) } \\
\end{array}$ & $\begin{array}{c}\text { n3 (N-terminal), } \\
\text { n (otherwise) } \\
\text { (GAFF v1.8) }\end{array}$ & $\begin{array}{l}\text { NH1 (CHARMM22 type, assigned as } \\
\text { in disarcosine in Mirijanian et al.) }\end{array}$ & NTOID (Weise \\
\hline $\mathrm{HN}^{\dagger}$ & $\begin{array}{l}\text { H (AMBER99SB- } \\
\text { ILDN) }\end{array}$ & hn (GAFF v1.8) & $\begin{array}{l}\text { HC (CHARMM22 type for hydrogen } \\
\text { in N terminal group) }\end{array}$ & $\begin{array}{l}\text { HGPAM1 (CGenFF type, } \\
\text { assigned from ParamChem) }\end{array}$ \\
\hline CB & $\begin{array}{l}\text { CT (AMBER99SB- } \\
\text { ILDN) }\end{array}$ & c3 (GAFF v1.8) & $\begin{array}{l}\text { CT2 (CHARMM22 type, assigned as } \\
\text { in disarcosine in Mirijanian et al.) }\end{array}$ & $\begin{array}{l}\text { CG311 (CGenFF type, } \\
\text { assigned from ParamChem) }\end{array}$ \\
\hline HB1 & $\begin{array}{l}\text { H1(AMBER99SB- } \\
\text { ILDN) }\end{array}$ & h1 (GAFF v1.8) & $\begin{array}{l}\text { HA (CHARMM22 type, assigned as } \\
\text { in disarcosine in Mirijanian et al.) }\end{array}$ & $\begin{array}{l}\text { HGA1 (CGenFF type, } \\
\text { assigned from ParamChem) }\end{array}$ \\
\hline $\mathrm{CD}^{*}$ & $\begin{array}{l}\text { CT (AMBER99SB- } \\
\text { ILDN) }\end{array}$ & c3 (GAFF v1.8) & $\begin{array}{l}\text { CTL2 (CHARMM22 type, Sezer et } \\
\text { al. C1L) }\end{array}$ & $\begin{array}{l}\text { CTL2 (CHARMM22 type, } \\
\text { Sezer et al. H1A) }\end{array}$ \\
\hline $\mathrm{HD}^{*}$ & $\begin{array}{c}\text { HC } \\
\text { (AMBER99SB- } \\
\text { ILDN) }\end{array}$ & hc (GAFF v1.8) & $\begin{array}{c}\text { HAL2 (CHARMM22 type, Sezer et } \\
\text { al. H1L) }\end{array}$ & $\begin{array}{l}\text { HAL2 (CHARMM22 type, } \\
\text { Sezer et al. H1A) }\end{array}$ \\
\hline
\end{tabular}




\begin{tabular}{|c|c|c|c|c|}
\hline CE* & $\begin{array}{c}\text { CT (AMBER99SB- } \\
\text { ILDN) }\end{array}$ & c3 (GAFF v1.8) & $\begin{array}{c}\text { CTL1 (CHARMM22 type, Sezer et } \\
\text { al. C1) }\end{array}$ & $\begin{array}{c}\text { CTL1 (CHARMM22 type, } \\
\text { Sezer et al. H1A) }\end{array}$ \\
\hline CF* & $\begin{array}{c}\text { CT (AMBER99SB- } \\
\text { ILDN) }\end{array}$ & c3 (GAFF v1.8) & $\begin{array}{c}\text { CTL3 (CHARMM22 type, Sezer et } \\
\text { al. C1A) }\end{array}$ & $\begin{array}{c}\text { CTL3 (CHARMM22 type, } \\
\text { Sezer et al. H1A) }\end{array}$ \\
\hline HF* & $\begin{array}{c}\text { HN (Stendardo et } \\
\text { al.) }\end{array}$ & hc (GAFF v1.8) & $\begin{array}{c}\text { HAL3 (CHARMM22 type, Sezer et } \\
\text { al. H1A) }\end{array}$ & $\begin{array}{c}\text { HAL3 (CHARMM22 type, } \\
\text { Sezer et al. H1A) }\end{array}$ \\
\hline NR & $\begin{array}{c}\text { NN (Stendardo et } \\
\text { al.) }\end{array}$ & n3 (GAFF v1.8) & NN (Sezer et al.) & NN (Sezer et al.) \\
\hline OR & $\begin{array}{c}\text { ON (Stendardo et } \\
\text { al.) }\end{array}$ & o (GAFF v1.8) & ON (Sezer et al.) & ON (Sezer et al.) \\
\hline
\end{tabular}

Table S11: Same as Table S8 but for the spin-label monomer. ${ }^{\dagger} \mathrm{HN}$ atom only appears in N-terminal spin-label. 


\section{S8. Forcefield parameterization details and missing forcefield parameters}

An overview of the forcefield parameterization are as follows:

MFTOID. We use MFTOID with the TIP3P water model modified for the CHARMM forcefield $^{22}$ (mTIP3P). We assign backbone atom types from Ref. [18 ]. We assign sidechain atom types and partial charges based on parameters for amino acids ${ }^{22,27}$ or using ParamChem ${ }^{24}$ (sections S6 and S7). We take nitroxide spin label parameters and charging from Ref. [20], a parameterization compatible with CHARMM forcefields. Partial charges and atom types for disarcosine are from Mirijanian et al.. ${ }^{18}$

GAFF- $\boldsymbol{\varphi}$. We use the GAFF- $\varphi$ forcefield with the $\mathrm{SPC} / \mathrm{E}^{28}$ water model. GAFF- $\varphi$ was originally parameterized with implicit solvent, but implicit solvent simulations show larger deviation from the DEER results than explicit solvent simulations (Fig. S31). We perform GAFF atom type assignment using AmberTools $16^{16}$ and GAFF version 1.8 and derive partial charges using the AM1-BCC model ${ }^{29}$ for the hydrophilic monomers. For the spin-labels we run electrostatic potential (ESP) calculations in Gaussian $16^{15}$ for dimers of the NME sidechain and the spin-label monomer with the B3LYP functional at the 6-311++G(d,p) level of theory and then use restrained electrostatic potential (RESP) fitting ${ }^{30}$ in AmberTools to localize the charge on each atom.

Modified AMBER99SB-ILDN. We use the modified AMBER99SB-ILDN forcefield with the SPC/E water model, as in the original study. ${ }^{31}$ We perform AMBER atom type assignment using AmberTools16 and derive partial charges for each hydrophilic monomer by computing the average partial charge on each atom of the monomer in homopolymers of length 8 after performing an ESP calculation in Gaussian 16 using Hartree-Fock level of theory with the 6-31G(d) basis set followed by RESP fitting. We take nitroxide spin label parameters from Ref. [21], a parameterization compatible with AMBER forcefields, omitting the lone-pair parameters, and assign the same partial charges as those used with the GAFF- $\varphi$ forcefield.

Modified CGenFF. We use the modified CGenFF forcefield with the mTIP3P water model. We assign backbone atom types from Ref. [32]. For the hydrophilic monomers, we assign sidechain atom types using ParamChem ${ }^{24,25}$ and assign the same partial charges as those used with MFTOID. We take nitroxide spin label parameters and charging from Ref. [20] and we assign remaining atom types using ParamChem.

In all cases, any additional missing parameters are derived based on analogous parameters in the forcefield. These missing parameters were derived from analogous parameters in the CGenFF4.1, ${ }^{23}$ CHARMM22, ${ }^{22}$ MFTOID, ${ }^{18}$ Weiser et al., ${ }^{26}$ and Sezer et al. ${ }^{20}$ forcefields, as described in the following tables.

\begin{tabular}{|c|c|c|c|}
\hline Atoms & $b_{i j}[\mathrm{~nm}]$ & $k_{i j}\left[\mathrm{~kJ} / \mathrm{mol} / \mathrm{nm}^{2}\right]$ & Source \\
\hline CT2-OG301 & 0.1415 & 301248 & CG321-OG301 in CGenFF \\
\hline CT3-OG301 & 0.1415 & 301248 & CG331-OG301 in CGenFF \\
\hline CT2-CTL2 & 0.1538 & 186188 & CT2-CT1 in CHARMM22 \\
\hline
\end{tabular}

Table S12: Additional parameters for harmonic bond potential in the MFTOID-based forcefield. The potential in GROMACS has the form $V\left(r_{i j}\right)=\frac{1}{2} k_{i j}\left(r_{i j}-b_{i j}\right)^{2}$. 


\begin{tabular}{|c|c|c|c|c|c|}
\hline Atoms & $\theta_{i j k}^{0}[\mathrm{deg}]$ & $\begin{array}{c}k_{i j k} \\
{\left[\mathrm{~kJ} / \mathrm{mol} / \mathrm{rad}^{2}\right]}\end{array}$ & $r_{i k}^{0}[\mathrm{~nm}]$ & $k_{i j k}^{U B}\left[\mathrm{~kJ} / \mathrm{mol} / \mathrm{nm}^{2}\right]$ & Source \\
\hline HC-NH1-TC & 123 & 284.512 & 0 & 0 & CG2O1-NG2S1-HGP1 in CGenFF \\
\hline CT2-CT2-OG301 & 111.5 & 376.56 & 0 & 0 & CG321-CG321-OG301 in CGenFF \\
\hline CT2-OG301-CT3 & 109.7 & 794.96 & 0 & 0 & CG321-OG301-CG331 in CGenFF \\
\hline OG301-CT2-HA & 108.89 & 384.0912 & 0 & 0 & OG301-CG321-HGA2 in CGenFF \\
\hline OG301-CT3-HA & 108.89 & 384.0912 & 0 & 0 & OG301-CG331-HGA3 in CGenFF \\
\hline HAL2-CTL2-CT2 & 110.1 & 221.752 & 0.2179 & 18853.104 & HA-CT2-CT2 in CHARMM22 \\
\hline CTL2-CT2-CTL2 & 113.5 & 488.2728 & 0.2561 & 9338.688 & CT1-CT2-CT1 in CHARMM22 \\
\hline CLT1-CTL2-CT2 & 113.5 & 488.2728 & 0.2561 & 9338.688 & CT1-CT2-CT1 in CHARMM22 \\
\hline HA-CT2-CTL2 & 110.1 & 288.696 & 0.2179 & 18853.104 & HA-CT1-CT2 in CHARMM22 \\
\hline NH1-CT2-CTL2 & 113.5 & 585.76 & 0 & 0 & NH1-CT1-CT2 in CHARMM22 \\
\hline
\end{tabular}

Table S13: Additional parameters for the Urey-Bradley angle potential in the MFTOID-based forcefield. The potential in GROMACS has the form $V\left(\theta_{i j k}\right)=\frac{1}{2} k_{i j k}\left(\theta_{i j k}-\theta_{i j k}^{0}\right)^{2}+\frac{1}{2} k_{i j k}^{U B}\left(r_{i k}-r_{i k}^{0}\right)^{2}$.

\begin{tabular}{|c|c|c|c|c|}
\hline Atoms & $\varphi_{s}[\mathrm{deg}]$ & $k[\mathrm{~kJ} / \mathrm{mol}]$ & $n$ & Source \\
\hline TC-CT2-NH1-CT2 & 0 & 7.5312 & 1 & TC-CT2-NH1-CT3 in Mirijanian et al. \\
\hline CT2-TC-NH1-CT2 & 180 & 7.5312 & 2 & CT2-TC-NH1-CT3 in Mirijanian et al. \\
\hline OG301-CT2-CT2-HA & 0 & 0.79496 & 3 & OG301-CG321-CG321-HGA2 in CGenFF \\
\hline CT2-CT2-OG301-CT3 & 0 & 2.38488 & 1 & CG321-CG321-OG301-CG331 in CGenFF \\
\hline CT2-CT2-OG301-CT3 & 0 & 1.21336 & 2 & CG321-CG321-OG301-CG331 in CGenFF \\
\hline CT2-CT2-OG301-CT3 & 0 & 1.79912 & 3 & CG321-CG321-OG301-CG331 in CGenFF \\
\hline HA-CT2-OG301-CT3 & 0 & 1.18826 & 3 & HGA-CG321-OG301-CG331 in CGenFF \\
\hline HA-CT3-OG301-CT2 & 0 & 1.18826 & 3 & HGA-CG321-OG301-CG321 in CGenFF \\
\hline NH1-CT2-CT2-OG301 & 180 & 0.66944 & 1 & CG331-CG321-CG321-OG301 in CGenFF \\
\hline NH1-CT2-CT2-OG301 & 0 & 1.63176 & 2 & CG331-CG321-CG321-OG301 in CGenFF \\
\hline TC-NH1-CT2-HA & 0 & 0 & 3 & HA-CT2-NH1-TC in Mirijanian et al. \\
\hline CT2-NH1-CT2-HA & 0 & 0 & 1 & HB-CT2-NH1-CT3 in Mirijanian et al. \\
\hline CT2-NH1-CT2-HB & 0 & 0 & 1 & HB-CT2-NH1-CT3 in Mirijanian et al. \\
\hline CT2-NH1-CT2-CT2 & 0 & 0.4184 & 3 & X-CT3-NH1-X in MFTOID \\
\hline CT2-TC-NH1-HC & 0 & 0 & 3 & $\begin{array}{l}\text { No obvious analogy; set to } 0 \text { because } \\
\text { involves a hydrogen }\end{array}$ \\
\hline O-TC-NH1-HC & 0 & 0 & 3 & $\begin{array}{l}\text { No obvious analogy; set to } 0 \text { because } \\
\text { involves a hydrogen }\end{array}$ \\
\hline NN-CTL1-CTL2-CT2 & 0 & 2.481112 & 3 & NN-CTL1-CTL2-CTL1 in Sezer et al. \\
\hline HA-CT2-CTL2-CTL1 & 0 & 0.8368 & 3 & X-CTL1-CTL2-X in CHARMM22 \\
\hline CTL2-CT2-CTL2-CTL1 & 0 & 0.8368 & 3 & $\mathrm{X}$-CTL1-CTL2-X in CHARMM22 \\
\hline NH1-CT2-CTL2-CTL1 & 0 & 0.8368 & 3 & $\mathrm{X}$-CTL1-CTL2-X in CHARMM22 \\
\hline CTL2-CT2-CTL2-HAL2 & 0 & 0.8368 & 3 & X-CTL1-CTL2-X in CHARMM22 \\
\hline HA-CT2-CTL2-HAL2 & 0 & 0.8368 & 3 & $\mathrm{X}$-CTL1-CTL2-X in CHARMM22 \\
\hline CT2-NH1-CT2-CTL2 & 0 & 0.4184 & 3 & CT2-NH1-CT2-CT2 here \\
\hline NH1-CT2-CTL2-HAL2 & 0 & 0 & 3 & $\begin{array}{l}\text { No obvious analogy; set to } 0 \text { because } \\
\text { involves a hydrogen }\end{array}$ \\
\hline
\end{tabular}

Table S14: Additional parameters for the proper dihedral potential in the MFTOID-based forcefield. The potential in GROMACS has the form $V\left(\varphi_{i j k l}\right)=k\left(1+\cos \left(n \varphi-\varphi_{s}\right)\right)$.

\begin{tabular}{|c|c|c|c|}
\hline Atoms & $\xi_{0}[\mathrm{deg}]$ & $k\left[\mathrm{~kJ} / \mathrm{mol} / \mathrm{rad}^{2}\right]$ & Source \\
\hline NH1-X-X-CT2 & 0 & 167.36 & NH1-X-X-CT3 in Mirijanian et al. \\
\hline
\end{tabular}

Table S15: Additional parameters for the improper dihedral potential in the MFTOID-based forcefield. The potential in GROMACS has the form $V\left(\xi_{i j k l}\right)=\frac{1}{2} k\left(\xi_{i j k l}-\xi_{0}\right)^{2}$. 


\begin{tabular}{|c|c|c|c|}
\hline Atoms & $\theta_{i j k}^{0}[\mathrm{deg}]$ & $k_{i j k}\left[\mathrm{kcal} / \mathrm{mol} / \mathrm{rad}^{2}\right]$ & Source \\
\hline O-C-CT & 120.4 & 80 & $\begin{array}{c}\text { Prakash input file (private communication); different } \\
\text { from Mirijanian et al. }\end{array}$ \\
\hline O-C-N & 122.9 & 80 & $\begin{array}{c}\text { Prakash input file (private communication); different } \\
\text { from Mirijanian et al. }\end{array}$ \\
\hline NT-CT-C & 107 & 50 & N-CT-C in Prakash input file (private communication) \\
\hline
\end{tabular}

Table S16: Additional parameters for the harmonic angle potential in the modified AMBER forcefield. The ones listed here are those that differed between the input file sent by Arushi Prakash (corresponding to the modified AMBER99SB-ILDN forcefield in Ref. [17]) and the original MFTOID forcefield from Mirijanian et al. (in these cases, we used the same parameters as the input file from Arushi Prakash), or are parameters that were added to the input file. The potential in AMBER has the form $V\left(\theta_{i j k}\right)=k_{i j k}\left(\theta_{i j k}-\theta_{i j k}^{0}\right)^{2}$.

\begin{tabular}{|c|c|c|c|c|}
\hline Atoms & $\varphi_{S}[\mathrm{deg}]$ & $k[\mathrm{kcal} / \mathrm{mol}]$ & $n$ & Source \\
\hline X-CT-N-X & 0 & 0 & 0 & Prakash input file (private communication) \\
\hline
\end{tabular}

Table S17: Additional parameters for the dihedral in the modified AMBER forcefield. The one listed here differed between the input file sent by Arushi Prakash (corresponding to the modified AMBER99SB-ILDN forcefield in Ref. [17]) and the original MFTOID forcefield from Mirijanian et al. (in this cases, we used the same parameters as the input file from Arushi Prakash). The potential in AMBER has the form $V\left(\varphi_{i j k l}\right)=k\left(1+\cos \left(n \varphi-\varphi_{s}\right)\right)$.

\begin{tabular}{|c|c|c|c|}
\hline Atoms & $b_{i j}[\mathrm{~nm}]$ & $k_{i j}\left[\mathrm{~kJ} / \mathrm{mol} / \mathrm{nm}^{2}\right]$ & Source \\
\hline CG311-CTL2 & 0.1538 & 186188 & CT2-CT1 in CHARMM22 \\
\hline NTOID-HGPAM1 & 0.098 & 338904 & NH1-HC in CHARMM22 \\
\hline NTOID-HGP1 & 0.098 & 338904 & NH1-HC in CHARMM22 \\
\hline CG311-NTOID & 0.143 & 267776 & CG311-NG2S1 in CGenFF \\
\hline
\end{tabular}

Table S18: Additional parameters for harmonic bond potential in the modified CGenFF-based forcefield. The potential in GROMACS has the form $V\left(r_{i j}\right)=\frac{1}{2} k_{i j}\left(r_{i j}-b_{i j}\right)^{2}$.

\begin{tabular}{|c|c|c|c|c|c|}
\hline Atoms & $\begin{array}{c}\theta_{i j k}^{0} \\
{[\mathrm{deg}]}\end{array}$ & $\begin{array}{c}k_{i j k} \\
{\left[\mathrm{~kJ} / \mathrm{mol} / \mathrm{rad}^{2}\right]}\end{array}$ & $r_{i k}^{0}[\mathrm{~nm}]$ & $\begin{array}{c}k_{i j k}^{U B} \\
{\left[\mathrm{~kJ} / \mathrm{mol} / \mathrm{nm}^{2}\right]}\end{array}$ & Source \\
\hline HAL2-CTL2-CG311 & 110.1 & 221.752 & 0.2179 & 18853.104 & HA-CT2-CT2 in CHARMM22 \\
\hline CTL2-CG311-CTL2 & 113.5 & 488.2728 & 0.2561 & 9338.688 & CT1-CT2-CT1 in CHARMM22 \\
\hline CLT1-CTL2-CG311 & 113.5 & 488.2728 & 0.2561 & 9338.688 & CT1-CT2-CT1 in CHARMM22 \\
\hline HGA1-CG311-CTL2 & 110.1 & 288.696 & 0.2179 & 18853.104 & HA-CT1-CT2 in CHARMM22 \\
\hline NTOID-CG311-CTL2 & 113.5 & 585.76 & 0 & 0 & NH1-CT1-CT2 in CHARMM22 \\
\hline $\begin{array}{c}\text { HGPAM1-NTOID- } \\
\text { CG321 }\end{array}$ & 109.5 & 251.04 & 0.2074 & 16736 & HC-NH3-CT2 in CHARMM22 \\
\hline $\begin{array}{c}\text { HGPAM1-NTOID- } \\
\text { CG311 }\end{array}$ & 109.5 & 251.04 & 0.2074 & 16736 & HC-NH3-CT1 in CHARMM22 \\
\hline HGP1-NTOID-HGP1 & 106.5 & 326.352 & 0 & 0 & HC-NH2-HC in CHARMM22 \\
\hline HGP1-NTOID-CG2O1 & 123 & 284.512 & 0 & 0 & HGP1-NG2S1-CG2O1 in CGenFF \\
\hline
\end{tabular}

Table S19: Additional parameters for the Urey-Bradley angle potential in the modified CGenFF-based forcefield. The potential in GROMACS has the form $V\left(\theta_{i j k}\right)=\frac{1}{2} k_{i j k}\left(\theta_{i j k}-\theta_{i j k}^{0}\right)^{2}+\frac{1}{2} k_{i j k}^{U B}\left(r_{i k}-r_{i k}^{0}\right)^{2}$. 


\begin{tabular}{|c|c|c|c|c|}
\hline Atoms & $\begin{array}{c}\varphi_{s} \\
{[\mathrm{deg}]}\end{array}$ & $k[\mathrm{~kJ} / \mathrm{mol}]$ & $n$ & Source \\
\hline NN-CTL1-CTL2-CG311 & 0 & 2.481112 & 3 & NN-CTL1-CTL2-CTL1 in Sezer et al. \\
\hline HGA1-CG311-CTL2-CTL1 & 0 & 0.8368 & 3 & X-CTL1-CTL2-X in CHARMM22 \\
\hline CTL2-CG311-CTL2-CTL1 & 0 & 0.8368 & 3 & X-CTL1-CTL2-X in CHARMM22 \\
\hline NTOID-CG311-CTL2-CTL1 & 0 & 0.8368 & 3 & X-CTL1-CTL2-X in CHARMM22 \\
\hline CTL2-CG311-CTL2-HAL2 & 0 & 0.8368 & 3 & X-CTL1-CTL2-X in CHARMM22 \\
\hline CG321-NTOID-CG311-CTL2 & 0 & 0.4184 & 3 & CT2-NH1-CT2-CT2 here \\
\hline HGA1-CG311-CTL2-HAL2 & 0 & 0.8368 & 3 & X-CTL1-CTL2-X in CHARMM22 \\
\hline NTOID-CG311-CTL2-HAL2 & 0 & 0 & 3 & $\begin{array}{l}\text { No obvious analogy; set to } 0 \text { because } \\
\text { involves a hydrogen }\end{array}$ \\
\hline CG321-CG2O1-NTOID-HGP1 & 0 & 0 & 3 & $\begin{array}{l}\text { No obvious analogy; set to } 0 \text { because } \\
\text { involves a hydrogen }\end{array}$ \\
\hline OG2D1-CG2O1-NTOID-HGP1 & 0 & 0 & 3 & $\begin{array}{l}\text { No obvious analogy; set to } 0 \text { because } \\
\text { involves a hydrogen }\end{array}$ \\
\hline HGPAM1-NTOID-CG321-CG321 & 0 & 0.4184 & 3 & $\mathrm{X}$-CT3-NH1-X in MFTOID \\
\hline HGPAM1-NTOID-CG321-HGA2 & 0 & 0.4184 & 3 & $\mathrm{X}$-CT3-NH1-X in MFTOID \\
\hline HGPAM1-NTOID-CG321-CG2O1 & 0 & 0.4184 & 3 & $\mathrm{X}-\mathrm{CT} 3-\mathrm{NH} 1-\mathrm{X}$ in MFTOID \\
\hline HGPAM1-NTOID-CG311-HGA1 & 0 & 0.4184 & 3 & X-CT3-NH1-X in MFTOID \\
\hline HGPAM1-NTOID-CG311-CTL2 & 0 & 0.4184 & 3 & X-CT3-NH1-X in MFTOID \\
\hline CG2O1-NTOID-CG311-HGA1 & 0 & 0.4184 & 3 & X-CT3-NH1-X in MFTOID \\
\hline CG2O1-NTOID-CG311-CTL2 & 0 & 0.4184 & 3 & X-CT3-NH1-X in MFTOID \\
\hline CG311-NTOID-CG321-CG2O1 & 0 & 3.3472 & 2 & $\begin{array}{l}\text { CG331-NTOID-CG321-CG2O1 in Weiser } \\
\text { and Santiso }\end{array}$ \\
\hline CG321-NTOID-CG321-CG2O1 & 0 & 3.3472 & 2 & $\begin{array}{c}\text { CG331-NTOID-CG321-CG2O1 in Weiser } \\
\text { and Santiso }\end{array}$ \\
\hline CG311-NTOID-CG321-HGA2 & 0 & 0 & 3 & $\begin{array}{l}\text { HGA2-CG321-NTOID-CG331 in Weiser } \\
\text { and Santiso }\end{array}$ \\
\hline CG321-NTOID-CG321-HGA2 & 0 & 0 & 3 & $\begin{array}{l}\text { HGA2-CG321-NTOID-CG331 in Weiser } \\
\text { and Santiso }\end{array}$ \\
\hline CG2O1-NTOID-CG321-CG321 & 0 & 0.4184 & 3 & X-CT3-NH1-X in MFTOID \\
\hline CG321-NTOID-CG321-CG321 & 0 & 0.4184 & 3 & $\mathrm{X}-\mathrm{CT} 3-\mathrm{NH} 1-\mathrm{X}$ in MFTOID \\
\hline CG321-NTOID-CG311-HGA1 & 0 & 0.4184 & 3 & X-CT3-NH1-X in MFTOID \\
\hline NTOID-CG321-CG321-OG301 & 180 & 0.66944 & 1 & CG331-CG321-CG321-OG301 in CGenFF \\
\hline NTOID-CG321-CG321-OG301 & 0 & 1.63176 & 2 & CG331-CG321-CG321-OG301 in CGenFF \\
\hline
\end{tabular}

Table S20: Additional parameters for the proper dihedral potential in the MFTOID-based forcefield. The potential in GROMACS has the form $V\left(\varphi_{i j k l}\right)=k\left(1+\cos \left(n \varphi-\varphi_{s}\right)\right)$. 


\section{S9. Time-domain simulations}

Simulated background-corrected DEER time-domain data, $F(t)$, can be computed, ${ }^{33}$ given a distance distribution $P(r)$, through convolution with the kernel, $\kappa(r, t)$ :

$$
\begin{gathered}
F(t)=1-\lambda\left(1-\sum_{i}^{N_{r}} \kappa\left(r_{i}, t\right) P\left(r_{i}\right) \Delta r\right) \\
\kappa(r, t)=\sqrt{\frac{\pi r^{3}}{6 C_{0} t}}\left[\cos \left(\frac{C_{0} t}{r^{3}}\right) \mathcal{C}\left(\sqrt{\frac{6 C_{0} t}{\pi r^{3}}}\right)+\sin \left(\frac{C_{0} t}{r^{3}}\right) \mathcal{S}\left(\sqrt{\frac{6 C_{0} t}{\pi r^{3}}}\right)\right]
\end{gathered}
$$

Here, $\lambda$ is the inversion efficiency (given by the DEER experiment), $N_{r}$ is the number of points in the discretized distance distribution, $\Delta r$ is the interval between distances in the distribution, $C_{0}$ is a constant and is equal to $3.27 \times 10^{5} \AA^{3} / \mu s$, and $\mathcal{C}$ and $\mathcal{S}$ are the Fresnel cosine and sine integrals. To compute the time-domain data from the simulated distance distributions, the latter are first linearly interpolated to reduce $\Delta r$, since insufficient points in the distance domain can lead to artificial high-frequency oscillations in the time-domain (Fig. S18). This interpolation should not have a significant impact on the computed time-domain signal (Fig. S18). For all simulated signals, the inversion efficiency, $\lambda$, in equation $\mathrm{S} 1$ is set to the experimentally derived value fitted in LongDistances (Table S21). The fitted distance distributions from SF-SVD are first modified by setting all negative values to zero and all values above the maximum end-to-end distance (Table S2) to zero, and renormalizing before computing the time-domain signal, in order to eliminate any obviously unphysical artifacts of the fit.

\begin{tabular}{|l|c|c|}
\hline Length & Modulation depth, $\boldsymbol{\lambda}$ & $\begin{array}{l}\text { Vertical shift for } \\
\text { Fig. 6 plot }\end{array}$ \\
\hline 9 & 0.072 & -0.27 \\
\hline 11 & 0.112968 & -0.14 \\
\hline 15 & 0.067 & -0.10 \\
\hline 19 & 0.082 & 0.0 \\
\hline
\end{tabular}

Table S21: The modulation depth, $\lambda$, in equation S1 is fitted in LongDistances. Here, it does not show a clear trend with respect to length in contrast to Kaminker et al. ${ }^{34}$ who showed that $\lambda$ decreases with an increase in the population of short distances. Here, our use of different observer pulse lengths for each experiment (in order to optimize the signal-to-noise ratio) results in different effective excitation bandwidths, which complicate the dependence of modulation depth on dipolar coupling. ${ }^{5}$
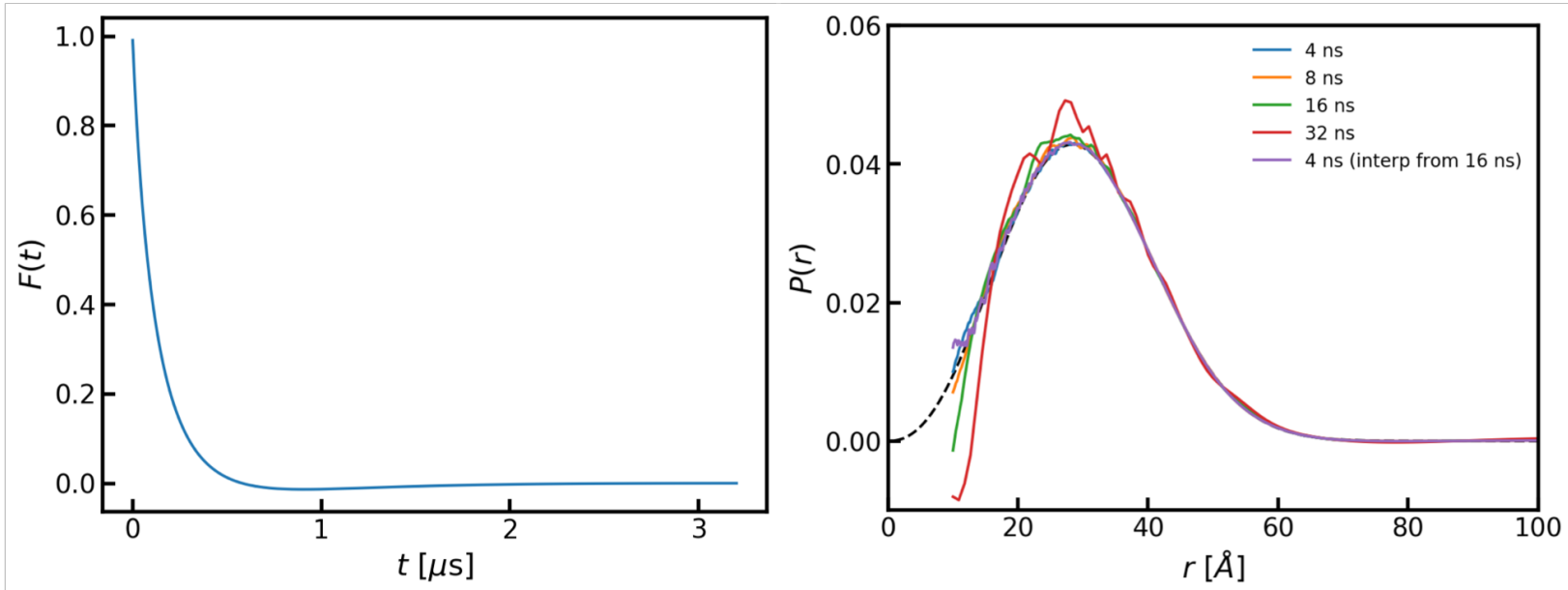

Figure S17: A time-domain signal (left) was computed at 1 ns resolution using equation $\mathrm{S} 1$ with $P(r)=$ $a_{1}(r / \tilde{r})^{\epsilon_{1}} \exp \left(-a_{2}(r / \tilde{r})^{\epsilon_{2}}\right) 4 \pi(r / \tilde{r})^{2}$ with $\epsilon_{1}=0.275, \epsilon_{2}=2.427, a_{1}=0.239, a_{2}=1.076, \tilde{r}=30 \AA$ for an excluded volume polymer distribution with mean end-to-end distance $30 \AA$ (black dashed line on right). The time- 
domain signal is then subsampled at intervals at varying lengths to give signals at different resolution (4ns, $8 \mathrm{~ns}, 16 \mathrm{~ns}$, 32ns). These signals are then fit using SF-SVD to produce the distance distributions at the right. As the time-resolution worsens, the deviation from the original distribution at shorter distances increases. Notably, fitting a signal with $4 \mathrm{~ns}$ resolution interpolated (linearly) from the $16 \mathrm{~ns}$ recovers the shorter distances, though we note that interpolation likely would not perform as well for a more realistic, noisy time-domain signal.
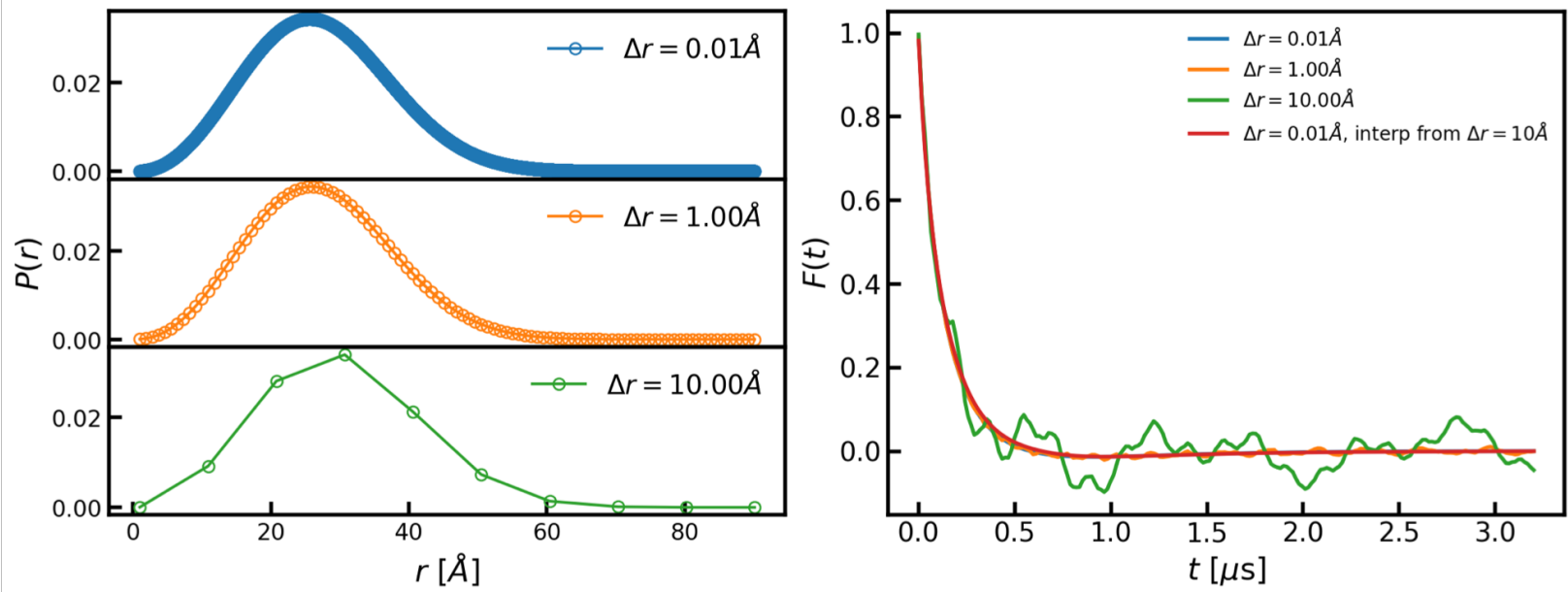

Figure S18: The same excluded volume polymer distribution as in Fig. S17 was used to generate distance distributions at varying distance resolution, $\Delta r$ (left). The time-domain signals were computed using equation S1. Large $\Delta r$ values lead to high-resolution frequencies in the time-domain signals, as the $\Delta r=10 \AA$ case demonstrates dramatically, and as is still visible for $\Delta r=1 \AA$. Linear interpolation of the distance distribution to reduce $\Delta r$ to $0.01 \AA$ removes these oscillations (red), and gives a time-domain signal approximately equal to the signal computed from original distance distribution with resolution $\Delta r=0.01 \AA$.

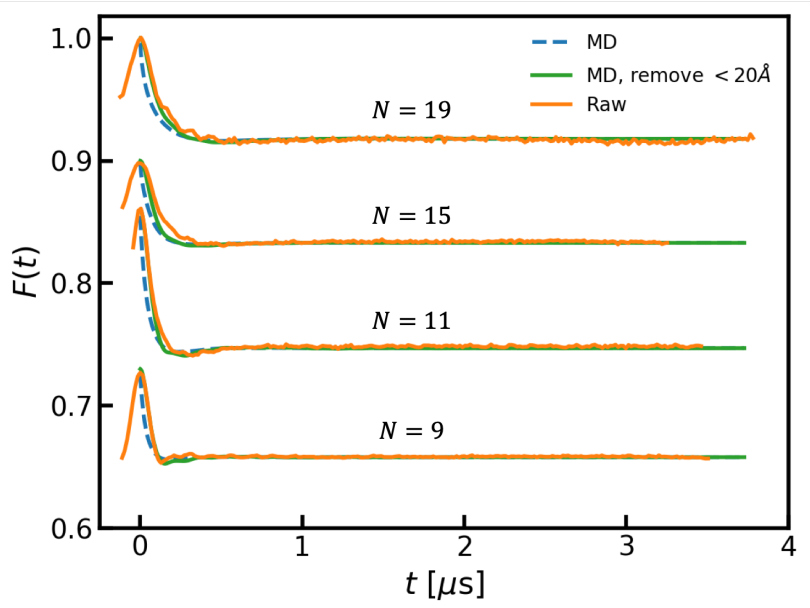

Figure S19: Removing the population below $20 \AA$ (green) shifts the low-time region of the back-calculated MD signal (derived from the $P\left(R_{e e}\right)$ computed using the MFTOID forcefield) closer to the raw signal, suggesting that the differences between the simulated and raw signals at short times could be due to DEER's lack of resolution in the < $20 \AA \AA$ regime. However, even if the population below $20 \AA$ is removed from the $N=9$ distance distribution before computation of the time-domain signal, the simulated signal still shows a slightly broader minimum than the raw signal, which is reflected in the narrower SF-SVD distribution in Fig. 5. 


\section{S10. Isomerization rate and correlation times of the $\omega$ dihedral}

When the $\omega$ dihedral potential is completely off $\left(\lambda_{e e}=0\right)$, the isomerization rate for the isomerizable $\omega$ dihedral in disarcosine is $\sim 150 \mathrm{~ns}^{-1}$. While measuring the isomerization rate in the unsoftened $\left(\lambda_{e e}=1\right)$ state is not tractable with the accessible simulation timescales, the isomerization rate as a function of $\lambda_{e e}$ follows approximately a logistic relationship (Fig. S20). Fitting the trend and extrapolating to $\lambda_{e e}=1$ leads to an estimated isomerization rate of $890 \mathrm{~s}^{-1}$, so that simulating in the fully softened $\left(\lambda_{e e}=0\right)$ state provides a speedup of 9 orders of magnitude. Experiments report even slower isomerization rates of $0.1 \mathrm{~s}^{-1}$ to $0.2 \mathrm{~s}^{-1}, 35$ which would indicate a speedup of 12 orders of magnitude.

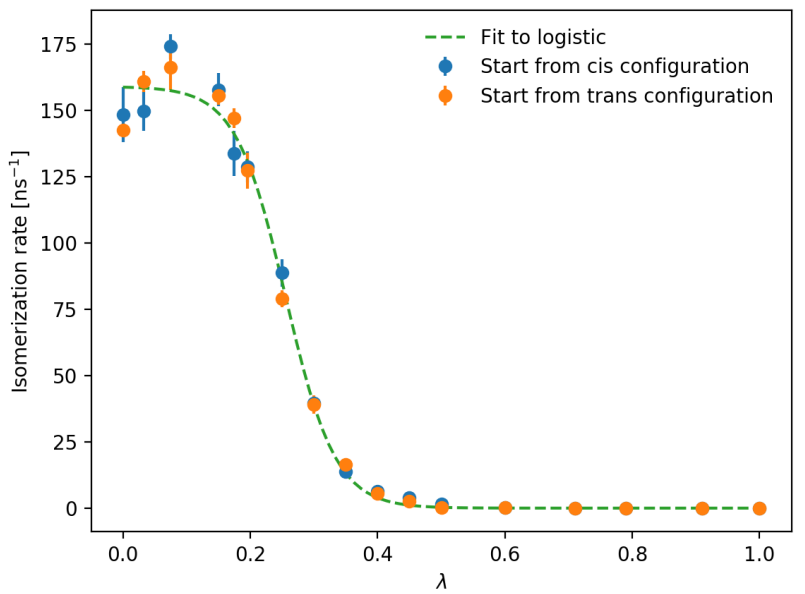

Figure S20: The isomerization rate is computed by simulating disarcosine (after an NVT equilibration for 500ps, followed by an NPT equilibration for 500ps, using a Monte Carlo (MC) barostat with 500fs between MC moves) at each $\lambda_{e e}$ state for $2 \mathrm{~ns}$, saving configurations every $0.2 \mathrm{ps}$, and counting the number of times the $\omega$ dihedral transitions between $-60<\omega<60$ and $\omega>120$ or $\omega<-120$. All settings (temperature, pressure, thermostat, barostat) are the same as for the expanded ensemble simulation. The parameters and partial charges are from Ref. [18], the MFTOID forcefield, and we simulate with mTIP3P water using the number of water molecules reported in Table S2. The measured isomerization rates, especially at small $\lambda_{e e}$, are underestimates, limited by the frequency of saving configurations, but the timescale associated with the maximum isomerization rate $\left(\sim 1 / 180 \mathrm{~ns}^{-1}=5.6 \mathrm{ps}\right)$ is more than 20 times the save frequency, so that the save frequency should not have a significant effect. The definition of an isomerization is also arbitrary and the measured isomerization rates change with the cutoffs used to define the cis and trans states which, at small $\lambda_{e e}$, are not the dominant $\omega$ states. The error bars show the standard error computed from three independent runs from the same initial cis or trans configurations and the points give the average over those three runs. In all cases, the measurements show an orders-of-magnitude increase in the isomerization rate as $\lambda_{e e}$ decreases. The trends are consistent regardless of whether the $\omega$ dihedral is initialized in the cis or trans configuration. The green dashed line shows a fit to a logistic relationship: $r=a /\left(1+\exp \left(-k\left(\lambda-\lambda_{0}\right)\right)\right.$.

In contrast, increasing the isomerization rate by increasing the temperature, as done in temperature replica exchange, requires very high temperatures. At $800 \mathrm{~K}$, the highest temperature used in Ref. [36], the isomerization rate is $5 \mathrm{~ns}^{-1}$, still slower than the isomerization rate at the fully softened state here, demonstrating the utility of a more targeted approach. 


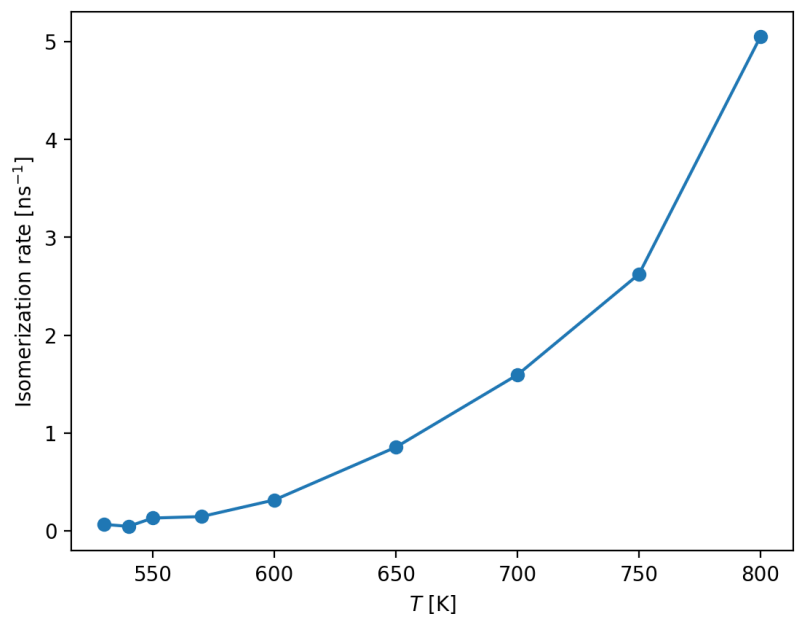

Figure S21: At each temperature, we equilibrate disarcosine in implicit solvent 100ps in the NVT ensemble and then run for 200ns in the NVT ensemble, saving configurations every $0.2 \mathrm{ps}$. The parameters and partial charges are from Ref. [18] (the MFTOID forcefield) and we simulate with the box size reported in Table S2. Implicit solvent simulations were run with the GBn2 solvation model ${ }^{37}$ without non-bonded interaction cutoffs using a Langevin thermostat with a friction coefficient of $1 p s^{-1}$. All other settings are the same as those in the expanded ensemble. We compute the isomerization rate by counting the number of times the $\omega$ dihedral transitions between $-60<\omega<60$ and $\omega>$ 120 or $\omega<-120$ and dividing by the total time of the production simulation (200ns).

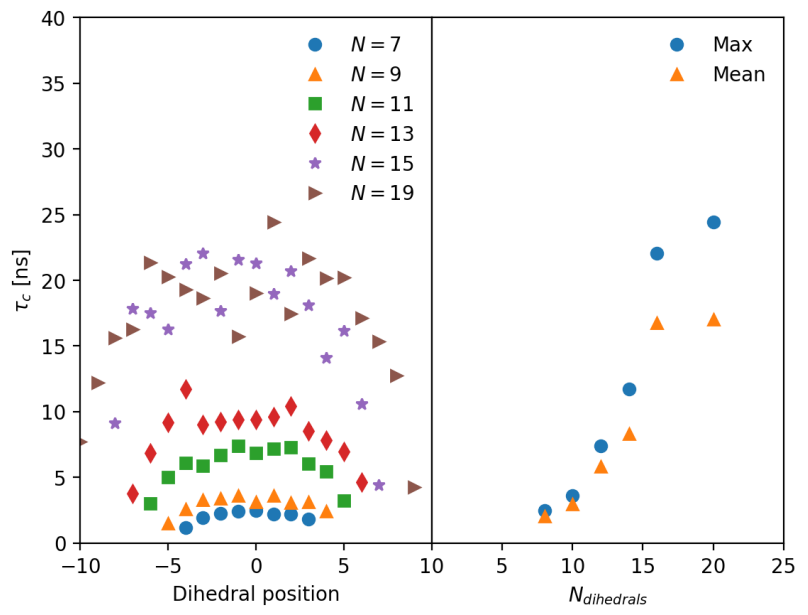

Figure S22: (Left) The correlation times for each dihedral in the hydrophilic polypeptoids, computed from the expanded-ensemble simulations using the MFTOID forcefield as the time constant from an exponential fit of the angle autocorrelation function defined in Ref. [38], are on the order of nanoseconds. Generally, correlation times increase with the length of the polypeptoid and distance of the dihedral from the end of the polypeptoid. Correlation times also depend on the distribution of $\lambda_{e e}$-states (having more states closer to $\lambda_{e e}=0$ reduces it, at the expense of simulating longer at more biased and thus less statistically significant states). (Right) The maximum and average $\omega$ correlation times for each polypeptoid length. 


\section{S11. Expanded ensemble simulation methodology, validation, and convergence}

For the equilibration period, we initialize all state weights to 0 and then use Self-Adjusted Mixture Sampling ${ }^{39}$ (SAMS) to sample across the $\lambda_{e e}$-states and a global weights update scheme ${ }^{39}$ to adjust state weights. When the histogram of states sampled is within $30 \%$ of flat, we estimate the free energy differences between each state and the unbiased state using the multistate Bennett acceptance ratio $(\mathrm{MBAR})^{40}$ and set the state weights to the free energy differences. After this equilibration period during which the weights are continuously updated, we fix the weights to those most recently computed with MBAR. We alternate between continuous weight updates with SAMS and simulating with fixed weights until the fixed weights are able to sample across all $\lambda_{e e^{-}}$ states. Convergence takes on the order of hundreds of nanoseconds (Fig. S24-S25). With the final fixed weights, we then run a production simulation with Metropolized independence sampling to sample states, saving configurations every $0.2 \mathrm{~ns}$.

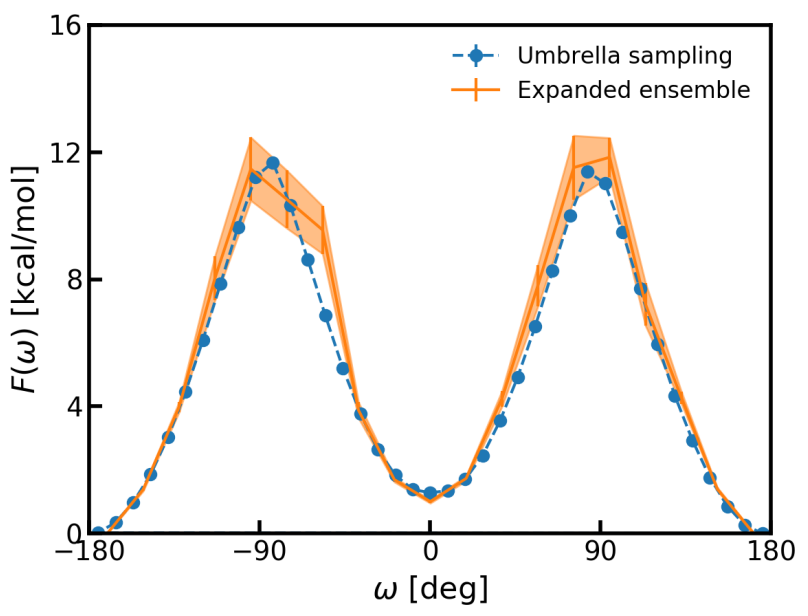

Figure S23: Validation of the expanded-ensemble methodology. The free energy landscape for rotation around the isomerizable amide bond of disarcosine computed using umbrella sampling and using the expanded ensemble method agree in the location of the local minima, demonstrating that the expanded ensemble method is able to reproduce the correct cis/trans populations. These simulations utilize the MFTOID forcefield, as an example, but we would expect agreement for any forcefield. Both plots give a $95 \%$ confidence interval computed with $\mathrm{MBAR}^{40,41}$ (section S13); for umbrella sampling, the uncertainty is smaller than the marker size.

For each hydrophilic polypeptoid, we use a set of $\lambda_{e e}$-states from $\lambda_{e e}=1$ to $\lambda_{e e}=0$. To compute the potential of mean force for rotation around the amide bond of disarcosine, we only use $\lambda_{e e^{-}}$ states from $\lambda_{e e}=1$ to $\lambda_{e e}=0.4$ in order to reduce the uncertainty around $\omega=90$ and $\omega=-90$, which are poorly sampled near equilibrium.

The weights for each $\lambda_{e e}$-state converge to within $\sim 0.5 k_{B} T$ of the free energy difference between the $\lambda_{e e}$-states (computed from the longer, production simulation) for $N=11$ within $600 \mathrm{~ns}$ and for $N=19$ within 800 ns (the trajectories of the weights for these two cases are shown as examples in Fig. S24 and S25, but we would expect this to be the typical behavior across all simulations). We iteratively adjust the weights using the two-stage SAMS scheme proposed in Ref. [39] with $\beta=$ 0.6 and the burn-in size, which determines the rate at which the weight updates decay, set to the number of trial state sampling steps for the histogram of states sampled to be within $30 \%$ of flat. 
We note that in the other equilibration simulations used to generate the weights used in this work, we typically set the burn-in size to infinity to allow for larger fluctuations in the weight updates to

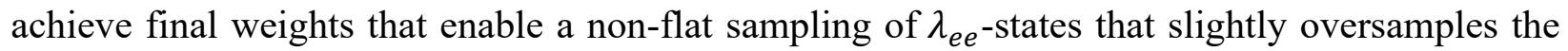
states closer to $\lambda_{e e}=1$ in order to achieve better statistics. This could be more rigorously accomplished by adjusting the mixture weights, $\pi$, as defined in Ref. [39].

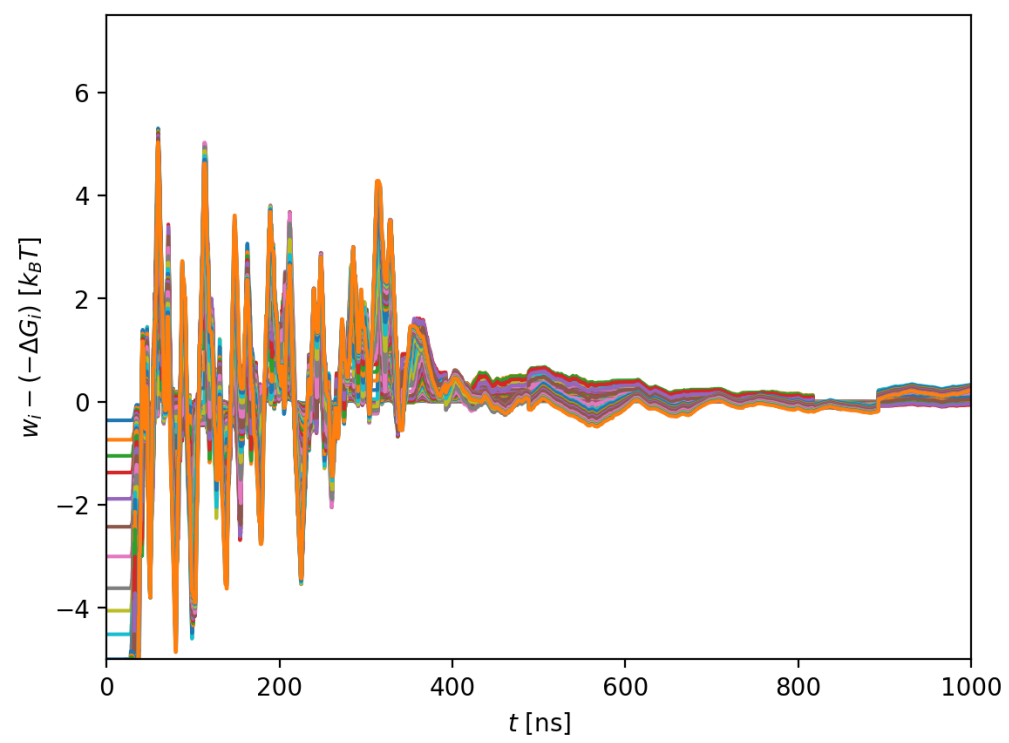

Figure S24: The difference between the estimated weight for each state, $w_{i}$, and the ideal weights, $-\Delta G_{i}$ (where $\Delta G_{i}=G\left(\lambda_{e e}=\lambda_{e e, i}\right)-G\left(\lambda_{e e}=1\right)$, the free energy difference between the unbiased state and state $i$, estimated using $\mathrm{MBAR}^{42}$ from the production simulation) converges to within $0.5 k_{B} T$ within $600 \mathrm{~ns}$ for $N=11$ with the MFTOID forcefield (we do not expect significant differences between forcefields besides the modified CGenFF for which convergence may be slower because more dihedrals are softened, leading to greater free energy differences between the unsoftened and fully softened states). The different colors correspond to the different states.

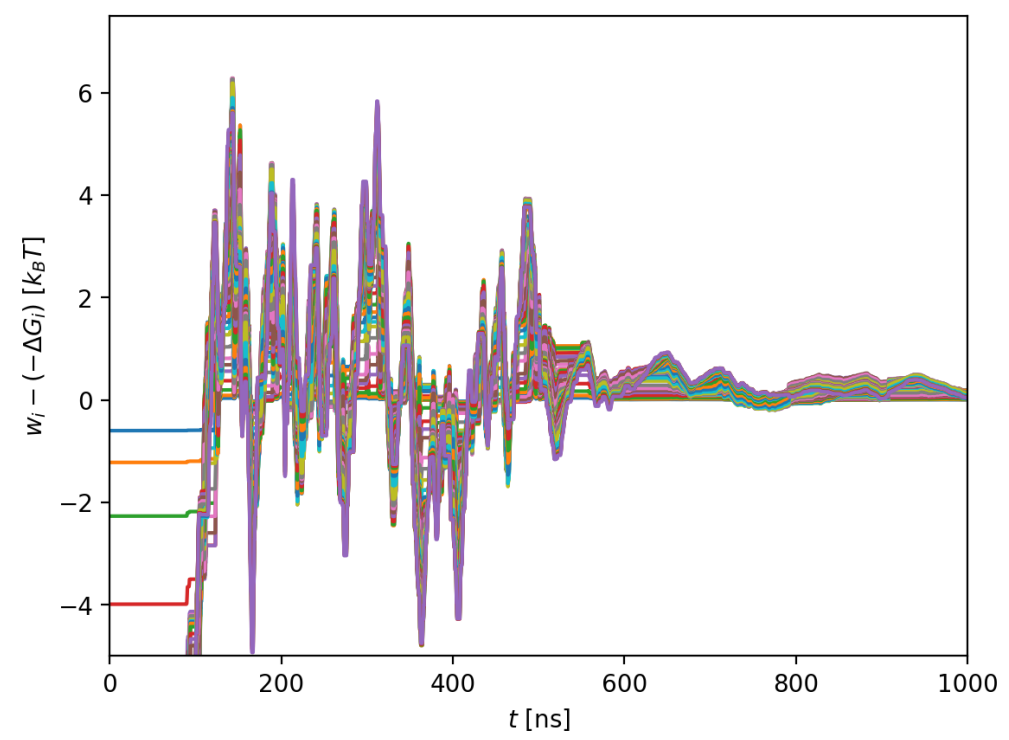

Figure S25: Same as Fig. S24 but for $N=19$ with the MFTOID forcefield. 


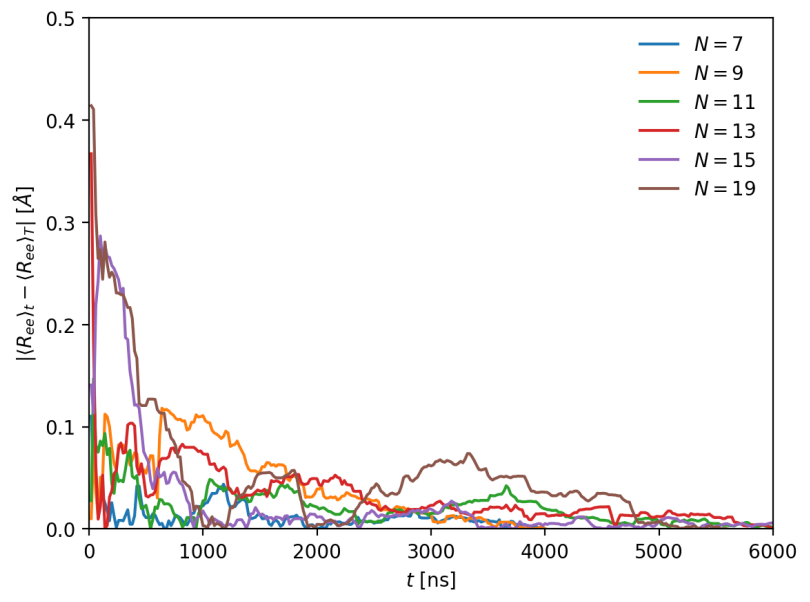

Figure S26: The convergence of the mean end-to-end distance to within $0.1 \AA$ of its final value takes on the order of $\mu \mathrm{s}$. The plotted values are the trajectories of the absolute value differences between the running and final mean endto-end distances for the different lengths with the MFTOID forcefield (we do not expect significant differences between forcefields besides the modified CGenFF for which convergence may be slower because more dihedrals are softened). 


\section{S12. Sampling of the $\phi$ dihedrals}

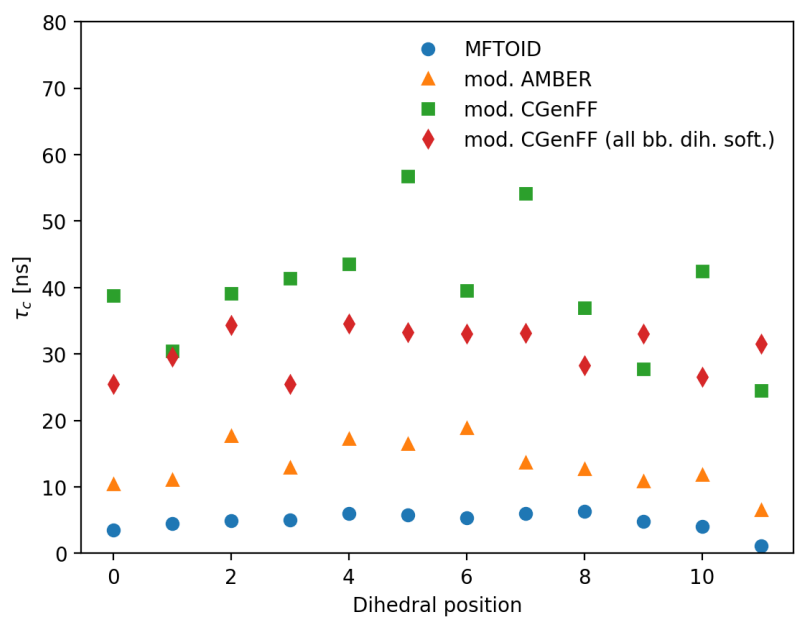

Figure S27: The correlation times for each dihedral in the $N=11$ polypeptoid, computed from the expandedensemble simulations as the time constant from an exponential fit of the angle autocorrelation function defined in Ref. [38]. For all points except the red diamonds, only the $\omega$ dihedral is softened. For the modified CGenFF forcefield, this results in poor sampling of isomerizations of the $\phi$ dihedral. Weiser and Santiso observed fewer than 10 transitions within $50 \mathrm{~ns}$ at $450 \mathrm{~K}$ for a trimer with methyl sidechains, and a decrease in frequency with sidechain size and polypeptoid length. ${ }^{26}$ Softening all backbone dihedrals (the $\omega, \varphi$, and $\phi$ dihedrals shown in Fig. 1) reduces the correlation time for the modified CGenFF forcefield. 


\section{S13. Uncertainty calculations for values derived from simulations}

\section{Free energy of rotation around disarcosine's amide bond}

For the expanded-ensemble calculation, the uncertainties shown in Fig. S23 are those returned from $\mathrm{MBAR}^{42}$ when reweighting the potential of mean force (PMF) for rotation around the amide bond, multiplied by 1.96 to give a $95 \%$ confidence interval. Note that for the MBARcomputed uncertainties to be accurate, the energies input must be from uncorrelated configurations. The statistical inefficiency estimated from the potential energies using the pymbar timeseries module is $304 \mathrm{ps}$ and configurations are saved every $200 \mathrm{ps}$, and so only every other configuration is used for the calculation of the PMF.

For the umbrella-sampling calculation, the uncertainties shown in Fig. S23 (smaller than the marker size) are also those returned from MBAR when reweighting the potential of mean force for rotation around the amide bond, multiplied by 1.96 to give a $95 \%$ confidence interval. The uncertainty is smaller than the marker size. Here, MBAR takes in all configurations, saved every $200 \mathrm{ps}$, so that the uncertainty is likely underestimated.

\section{End-to-end distance distributions}

First, the statistical inefficiency, $g$, is computed using the pymbar timeseries module ${ }^{42,43}$ using the maximum $g$ computed for the trajectories of the potential energy, backbone end-to-end distance, and spin-label end-to-end distance. The number of independent samples, $N_{s, \text { uncorr }}$ is estimated to be the total number of configurations divided by the statistical inefficiency. MBAR is used to estimate the weights for each configuration. Then, 1000 distributions are generated by repeatedly uniformly sampling $N_{s, \text { uncorr }}$ configurations from the entire trajectory with replacement and computing the distance distribution with each configuration weighted according to the MBAR weights. These 1000 distributions are averaged to produce a mean distance distribution and the uncertainties plotted in Figs. 4, 5, 8 are a $68 \%$ confidence interval. The uncertainty in the MBAR-dervied weights is assumed to be small relative to the uncertainty due to limited sampling of the end-to-end distance.

\section{Number of independent and statistically significant samples}

We estimate the average length of simulation time between independent and statistically significant samples using two different methods. Results from both are shown in Fig. S28 and are reasonably consistent. In the first method, we compute the effective sample number, $N_{\text {eff }}$, the number of samples that contribute to an average at the unbiased state, or the number of "significant" samples, for the entire dataset using MBAR. Then, $N_{e f f} / N$ is the fraction of samples that are significant. If we assume that each sample is equally likely to be significant, then the time in between independent and significant samples can be approximated as $g N / N_{\text {eff }}$, where $g$ is the statistical inefficiency defined as above. For the second method, we repeatedly draw a random sample of size $N_{s, \text { uncorr }}$, defined as above. For each sample, which should now contain, on average,

only independent configurations, we compute $N_{\text {eff,sample }}$. Then, on average, the simulation time between independent and significant samples is $T / N_{\text {eff,sample }}$ where $T$ is the total length of the simulation. 


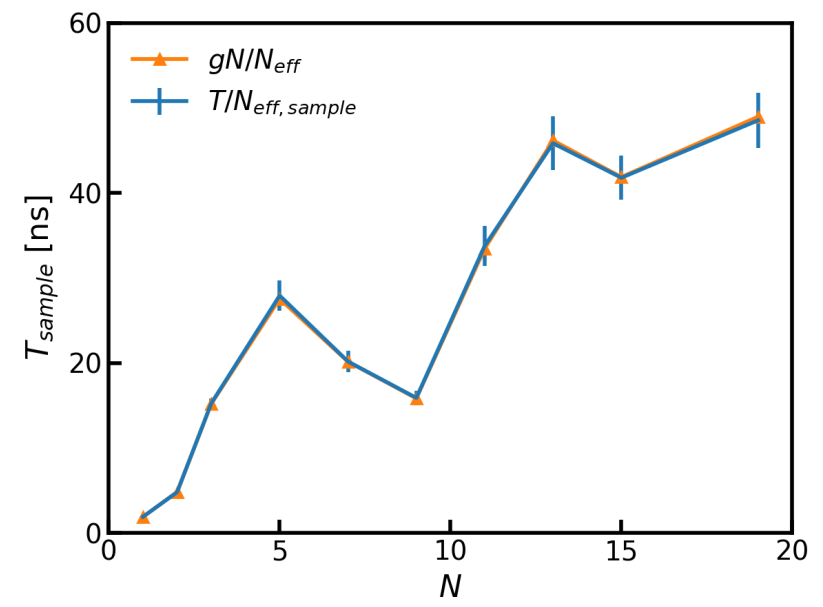

Figure S28: Time in between independent and statistically significant samples. Estimates using two different methods described above for different polypeptoid lengths with the MFTOID forcefield (we do not expect significant differences between forcefields besides the modified CGenFF where more dihedrals are softened) are consistent and increase with the number of monomers $N$. However, the quantity also depends on the $\lambda_{e e}$ states and state weights and thus will not necessarily be monotonic with $N$.

\section{Distances between probability distributions}

The same procedure as used in the computation of the uncertainty in the end-to-end distance distribution is used to generate 1000 distance distributions. The Shannon-Jensen distance and the Wasserstein distance are computed between each simulated distance distribution and the DEER distribution, yielding 1000 distances. The reported value is the mean of these values and the reported intervals give $95 \%$ confidence intervals. The contribution to the uncertainty due to uncertainty in the DEER distribution is assumed to be small and not computed.

\section{Mean end-to-end distances}

As in the computation of the uncertainty in the end-to-end distance distribution, the number of independent samples is computed from the statistical inefficiency. MBAR is used to estimate the weights for each configuration. Then, 1000 means are computed by repeatedly uniformly sampling $N_{s, \text { uncorr }}$ configurations from the entire trajectory with replacement and computing the mean distance with each configuration weighted according to the MBAR weights. The 1000 means are averaged and the error bars in Fig. 7 show a 95\% confidence interval.

The same procedure is followed to compute the uncertainty in the mean within the cw-EPR optimal region for the $N=3$ polypeptoid, whose distribution is shown in Fig. 8 . The only change is that the weights for the configurations with end-to-end distances outside the optimal region are set to 0 .

\section{Scaling exponents}

The reported uncertainties of the scaling exponent, prefactors, and shift factors are one standard deviation estimated from the curve_fit function in scipy.optimize. These uncertainties do not account for the uncertainty in the estimates of the individual values or the propagated uncertainties for values held constant during the fit (e.g. the prefactor in the scaling of the spinlabel end-to-end distance or the shift factor in the scaling of the DEER distances); they just reflect the uncertainty of the fit. 


\section{S14. Umbrella sampling details}

Forty simulations of disarcosine were run restraining the $\omega$ dihedral to some target value, evenly spaced between $-180^{\circ}$ and $180^{\circ}$. The parameters and partial charges are from Ref. [18] (the MFTOID forcefield) and we simulate with mTIP3P water using the number of water molecules reported in Table S2. A harmonic potential, $U=k\left(\omega-\omega_{0}\right)^{2}$ with force constant $k=$ $100 \mathrm{~kJ} / \mathrm{mol}$ and $\omega$ in radians was applied to restrain the dihedral. Prior to the umbrella sampling run, we run an NVT equilibration for 500ps, followed by an NPT equilibration for 500ps, using a Monte Carlo (MC) barostat with 500fs between MC moves. For the production runs, we use the same conditions (temperature, pressure, thermostat, barostat) as the expanded ensemble simulation. Each umbrella simulation is run for $20 \mathrm{~ns}$, with configurations saved every $20 \mathrm{ps}$. The free energy around the $\omega$ dihedral is reconstructed using MBAR.

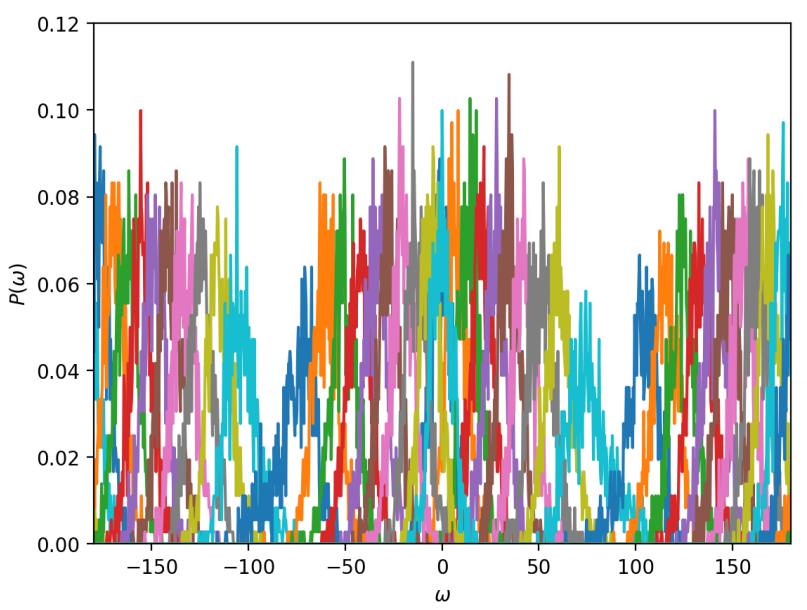

Figure S29: The distribution of $\omega$ dihedrals accessed in each separate umbrella sampling simulation. 
S15. Alternative measures of the distance between distributions

\begin{tabular}{|l|l|l|l|l|}
\hline Forcefield & $\begin{array}{l}\text { JS with SF- } \\
\text { SVD (reported } \\
\text { in main text) }\end{array}$ & $\begin{array}{l}\text { JS with } \\
\text { LongDistances }\end{array}$ & $\begin{array}{l}\text { Wasserstein } \\
\text { with SF-SVD }\end{array}$ & $\begin{array}{l}\text { Wasserstein } \\
\text { with } \\
\text { LongDistances }\end{array}$ \\
\hline MFTOID & $0.16(0.11,0.22)$ & $0.16(0.11,0.21)$ & $1.5(0.7,2.3)$ & $1.5(0.7,2.3)$ \\
\hline GAFF- $\varphi$ & $0.19(0.13,0.26)$ & $0.19(0.13,0.27)$ & $1.1(0.6,2.1)$ & $1.2(0.6,2.1)$ \\
\hline $\begin{array}{l}\text { Modified } \\
\text { AMBER }\end{array}$ & $0.30(0.21,0.39)$ & $0.30(0.21,0.40)$ & $3.0(1.6,4.3)$ & $3.0(1.6,4.3)$ \\
\hline $\begin{array}{l}\text { Modified } \\
\text { CGenFF }\end{array}$ & $0.22(0.15,0.29)$ & $0.22(0.15,0.29)$ & $2.2(1.2,3.2)$ & $2.3(1.2,3.2)$ \\
\hline
\end{tabular}

Table S22: The first Wasserstein distance or the earth mover's distance ${ }^{44}$ measures the distance between two probability distributions. The calculation gives similar trends compared to the Jensen-Shannon distance (the GAFF- $\phi$ and MFTOID forcefields are flipped, but this difference is well within the uncertainty). Computing both distances using the DEER distribution fit from LongDistances (as opposed to SF-SVD) gives very similar results, as the two DEER distributions are very similar above $20 \AA$ (Fig. S30). These metrics are computed in the DEER-optimal (> 20Å) region only. Prior to computation, in order to eliminate any unphysical artifacts, the DEER distribution is set to 0 above the maximum possible $R_{e e}(45.4 \AA$, see Table S2) and negative values in the distributions are also set to 0 . The DEER distribution is then renormalized. Parentheses give $95 \%$ confidence intervals, computed as described in section S13.

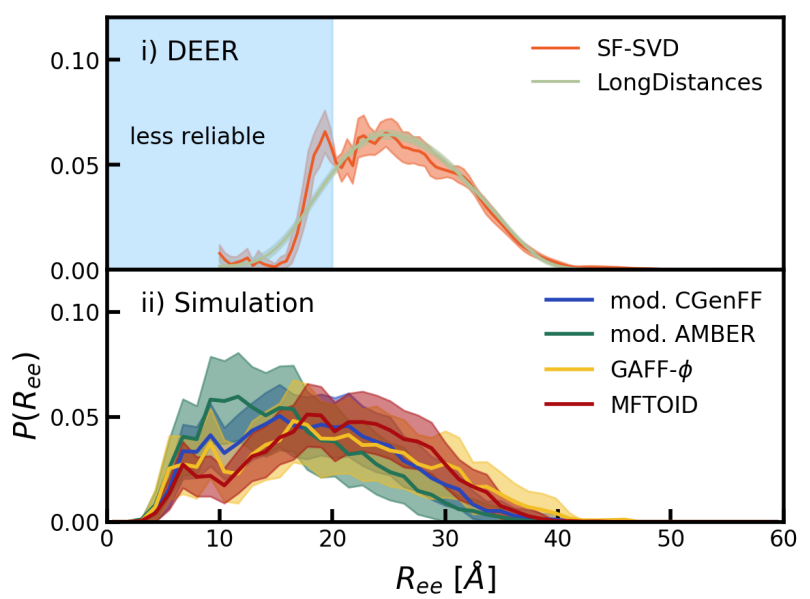

Figure S30: Same as Fig. 4, but with the DEER distribution fit using LongDistances (regularized inversion) as well. 


\section{S16. Impact of water model}

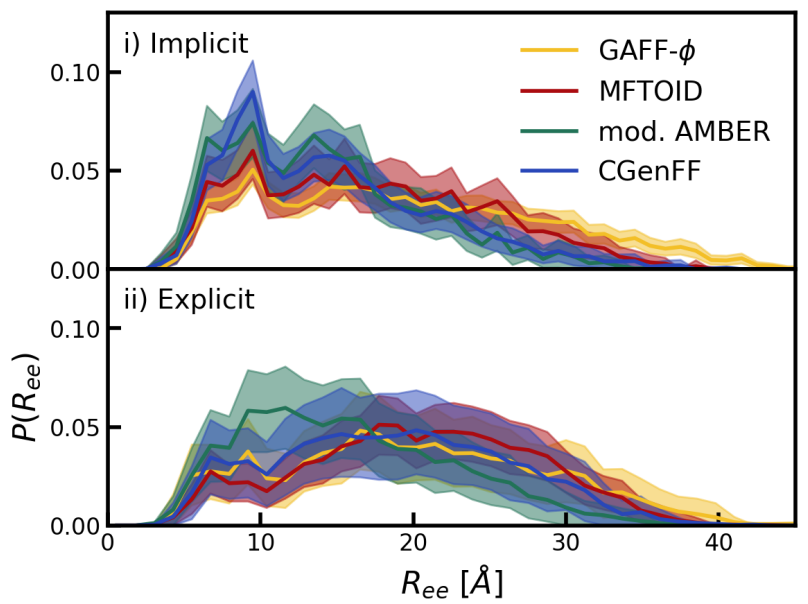

Figure S31: End-to-end distance distributions for $N=11$ computed for each polypeptoid forcefield in implicit solvent (top) compared to those in explicit solvent (bottom, same as the distributions in Fig. 4). Implicit solvent simulations were run with the GBn2 solvation model ${ }^{37}$ without non-bonded interaction cutoffs using a Langevin thermostat with a friction coefficient of $1 p s^{-1}$. Generally, they give more collapsed conformations compared to the explicit solvent.

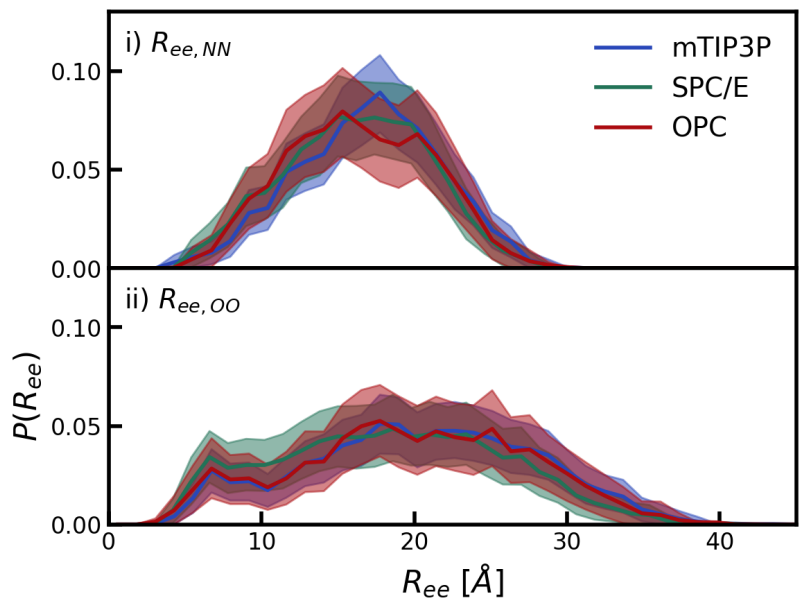

Figure S32: Backbone (top) and spin-label (bottom) end-to-end distance distributions computed for $N=11$ for the MFTOID forcefield with the modified TIP3P, SPC/E, and OPC4 ${ }^{45,46}$ water models. There is little deviation between water models, only a slight shift to lower spin-label end-to-end distances for the SPC/E model. 


\section{S17. Dihedral preferences for hydrophilic polypeptoid and disarcosine}

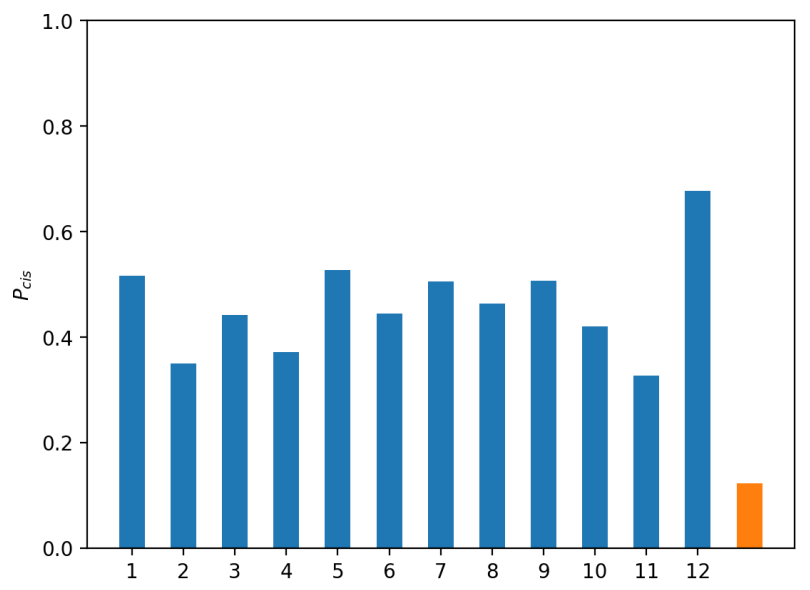

Figure S33: The probability distribution for rotation around each $\omega$ dihedral in the backbone of $N=11$ and also around the $\omega$ dihedral in disarcosine is reweighted from the simulations to the unbiased state using MBAR and the cis preference is computed as the fraction of the distribution between $\omega>90$ and $\omega<90$. These calculations are from the expanded ensemble simulations performed for $N=11$ and disarcosine using the MFTOID forcefield. For the hydrophilic polypeptoid, the cis preference for each dihedral with the nitrogen in a hydrophilic monomer is, on average, 0.44. The cis preference for the dihedral with the nitrogen in the C-terminal spin-label monomer is higher, at 0.68 , consistent with evidence that bulky groups directly attached to the backbone nitrogen lead to a stronger cis preference. ${ }^{47}$ For the same reason, the cis preference for disarcosine isomerizable $\omega$ dihedral is much lower, at 0.12 , since the sidechain is much smaller (a methyl group). Since the most extended conformation places all dihedrals in the trans configuration, a stronger trans preference in disarcosine could bias the conformations towards longer end-toend distances. 


\section{S18. Comparison of labeled and unlabeled molecules}

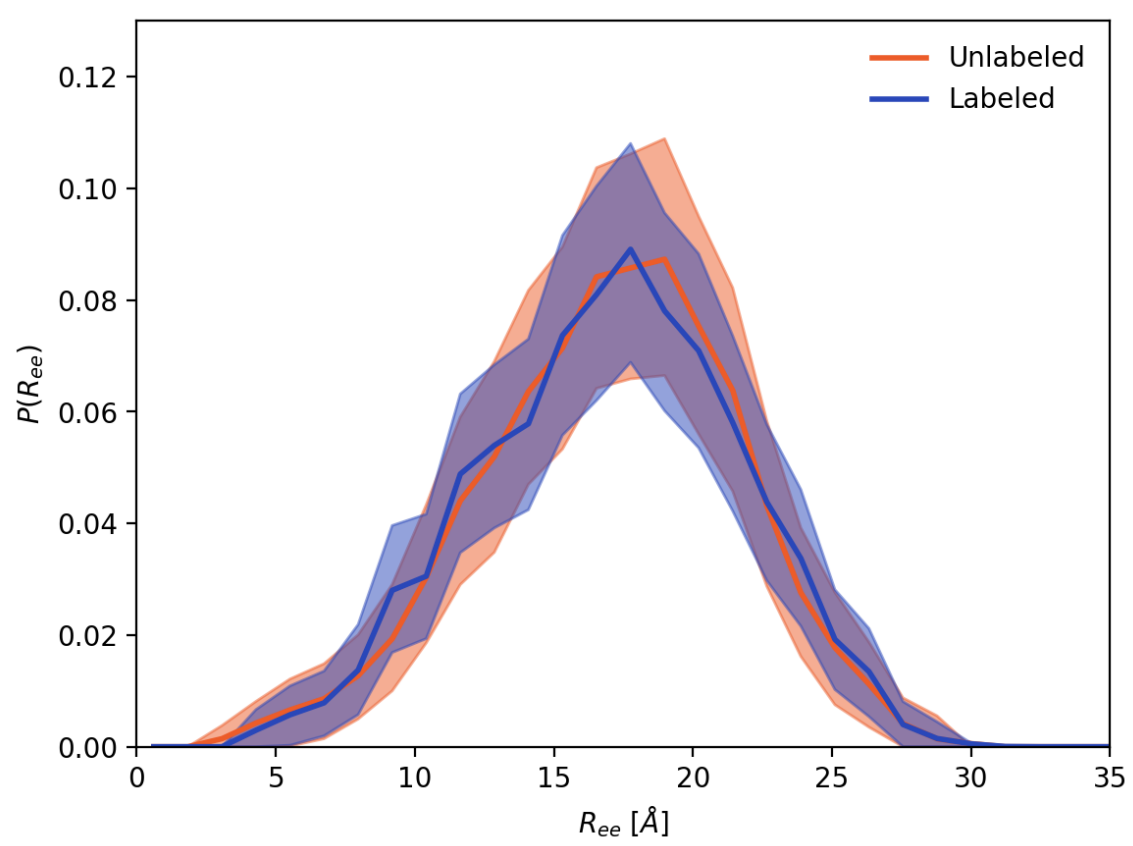

Figure S34: The backbone end-to-end distance distributions, $R_{e e, N N}$, for the $N=11$ polypeptoid terminated with spin labels ("Labeled") and without spin labels ("Unlabeled"), both simulated using the MFTOID forcefield. Their agreement suggests that the spin labels do not significantly perturb the underlying backbone conformational distribution of the polypeptoids. 


\section{S19. Comparison to theoretical form of $P\left(R_{e e}\right)$}

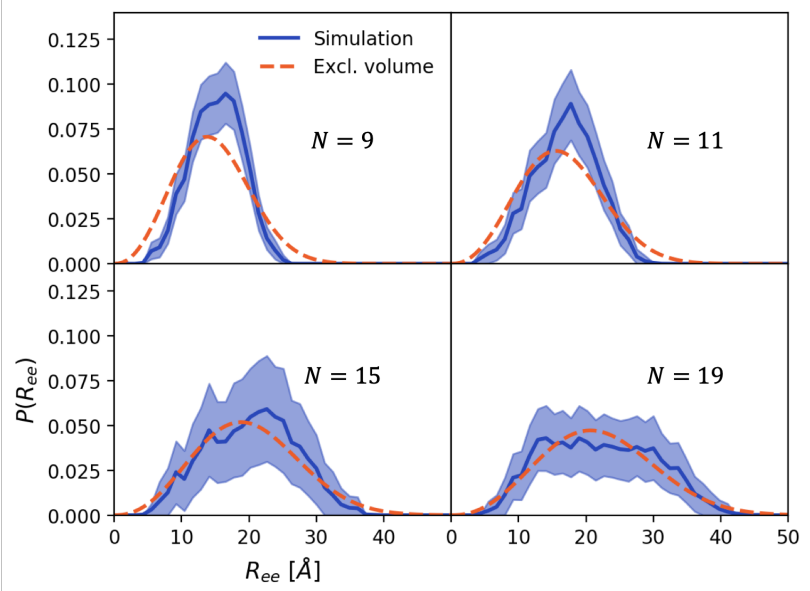

Figure S35: The blue lines give the $P\left(R_{e e, N N}\right)$, the end-to-end distance distribution measured from simulation (with the MFTOID forcefield) in between terminal nitrogens on the polypeptoid backbone (Fig. 7 inset) with shaded regions showing a $68 \%$ confidence interval, computed as described in section S13. The orange, dashed lines show a theoretical $P\left(R_{e e}\right)$ given by: $P\left(R_{e e}\right)=a_{1} x^{\varepsilon_{1}} \exp \left(-a_{2} x^{\varepsilon_{2}}\right) 4 \pi x^{2}$ where $x=R_{e e} /\left\langle R_{e e}^{2}\right\rangle^{1 / 2}{ }^{48}$ and $\varepsilon_{1}=0.269, \varepsilon_{2}=2.427$, $a_{1}=0.299$, and $a_{2}=1.269$. At longer lengths, the theoretical distribution is consistent with the simulated distribution, suggesting that the polypeptoid backbone follows excluded volume scaling. 


\section{S20. Scaling of mean end-to-end distances from LongDistances}

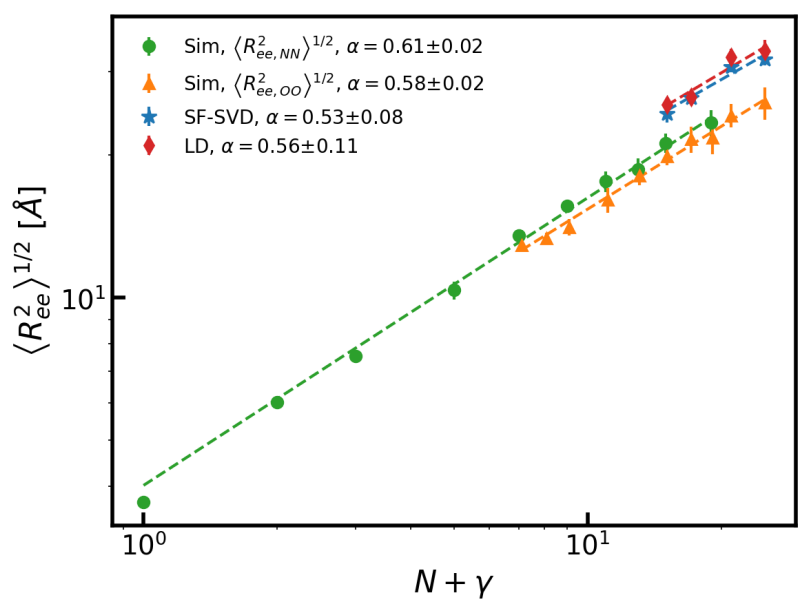

Figure S36: The mean end-to-end distances computed from the DEER distributions fit using LongDistances $(\alpha=$ $0.56 \pm 0.11)$ are consistent with those from the SF-SVD-fitted distributions $(\alpha=0.53 \pm 0.08)$. The prefactors are also within uncertainty ( $b=5.6 \pm 1.9$ and $b=5.9 \pm 1.5$, respectively). Both sets are fit using the same shift factor derived from the simulated distributions $(\gamma=6.0)$. The simulated mean end-to-end distances are the same as those reported in Fig. 7 of the main text. 


\section{S21. Length scaling for MFTOID and modified CGenFF forcefields}

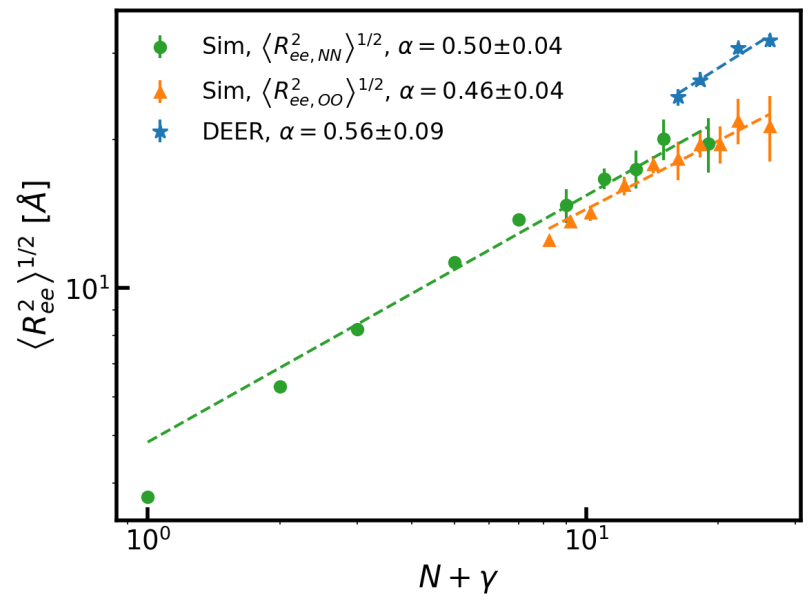

Figure S37: The scaling exponents are fit for the mean end-to-end distances computed from the modified CGenFF forcefield the same way as reported in the main text for the MFTOID forcefield. The backbone end-to-end distances, $\left\langle R_{e e, N N}^{2}\right\rangle^{1 / 2}$ are fit to the power law $b N^{\alpha}$, resulting in a scaling exponent of $\alpha=0.50 \pm 0.04$ and a prefactor of $b=$ $4.8 \pm 0.4$. The spin-label end-to-end distances, $\left\langle R_{e e, o o}^{2}\right\rangle^{1 / 2}$, are then fit to a shifted power law, $b(N+\gamma)^{\alpha}$ with $b$ set to 4.8 , yielding scaling exponent of $\alpha=0.46 \pm 0.04$ and a shift factor of $\gamma=7.2$. When the DEER data is fit to $b(N+\gamma)^{\alpha}$ with this shift factor, the resulting scaling exponent is $\alpha=0.56 \pm 0.09$ and the prefactor is $b=5.2 \pm 1.4$. The mean end-to-end distances computing using the modified CGenFF forcefield follow approximately ideal scaling ( $\alpha=0.5)$, which is not consistent with the excluded volume scaling predicted from the DEER data, from MFTOID for the same hydrophilic polypeptoids, or from Ref. [49] for polysarcosine.

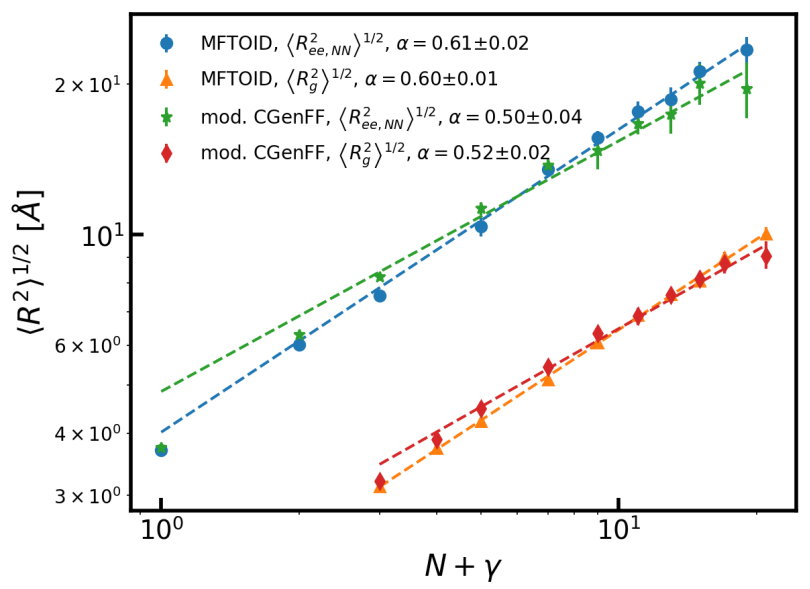

Figure S38: For both the MFTOID and the modified CGenFF forcefields, the scaling exponent of the backbone endto-end distances, $\left\langle R_{e e, N N}^{2}\right\rangle^{1 / 2}$, is consistent with the scaling of the radius of gyration, computed as the radius of gyration of the nitrogen atoms in the backbone. $N$ is the number of hydrophilic monomers, so $\gamma=0$ for the backbone distances, which are computed between the nitrogens of the first hydrophilic monomer and the C-terminal spin-label, and $\gamma=2$ for the radii of gyration, which are computed including the nitrogens on the N-terminal spin-label and the amino group at the C-terminus. The radius of gyration thus also suggests that the modified CGenFF forcefield gives a smaller scaling exponent, closer to ideal scaling, than the MFTOID forcefield. 


\section{S22. Removing configurations at short distances}

The simulated distributions reveal a peak at low end-to-end distance (approximately $8 \AA$ ) that decreases with polypeptoid length and that is likely due to spin-label interaction. This shortdistance feature is below the accessible region for DEER (within the shaded blue box in Fig. 5) and has been previously observed in simulations of polyethylene oxide labeled with the same spinlabel, but using a different spin-label model, ${ }^{7}$ suggesting the aggregation occurs independently of the chosen forcefield. To eliminate the contribution from spin-label interaction that is not captured by DEER, we remove the conformations at short distances in which the spin labels are oriented towards each other. To separate out this contribution, we cluster the configurations to generate groups with the same spin-label conformations. This clustering is performed only on the spin-label heavy atom positions for conformations with a spin-label end-to-end distance below $20 \AA$ and is based on the optimally aligned root-mean-squared distance ${ }^{50}$ between conformations of only the spin-label heavy atoms, using an RMSD cutoff of 2.5Å. Fig. S39 shows an example of this clustering for the $N=11$ polypeptoid from the expanded ensemble simulation with the MFTOID forcefield.

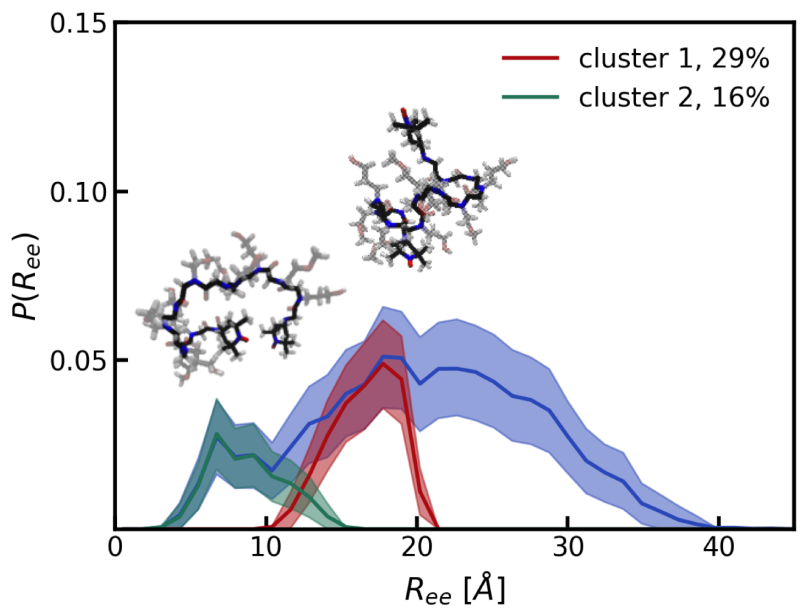

Figure S39: Clustering the spin-label conformations allows identification of the conformations in which the spin labels have aggregated. Here, in the cluster centered at the lowest end-to-end distance (green), the spin labels are oriented towards each other.

We recompute the end-to-end distance distribution without the configurations in the clusters at the lowest end-to-end distance, effectively subtracting out the peak at small end-to-end distances $(\sim 8 \AA)$ but preserving the distributions at higher end-to-end distances. 


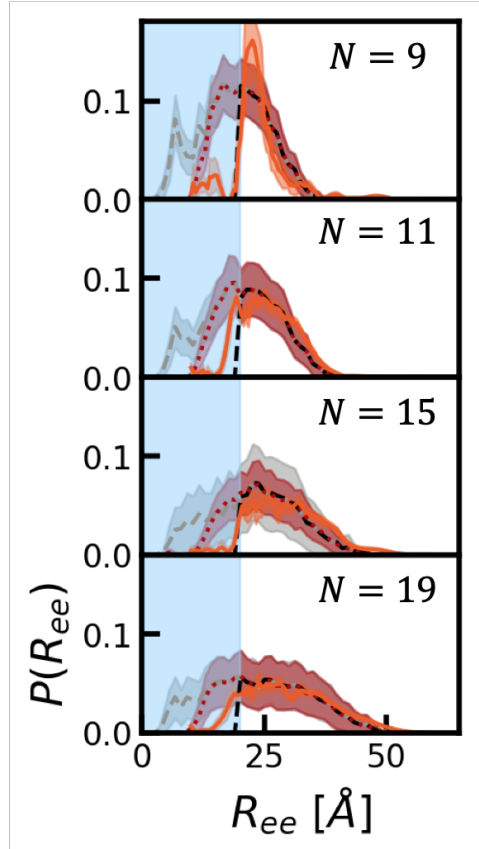

Figure S40: The short-distance peak in the unmodified distribution (dashed lines, same as those in Fig. 5 in the main text, computed from expanded ensemble simulations with the MFTOID forcefield) has been subtracted out of the modified distribution (dotted lines) to approximately account for the inability of DEER to resolve shorter distances where dipolar coupling occurs. Both the unmodified and modified end-to-end distributions show a shift to longer distances and broadening with increasing number of monomers. All distributions are normalized so that their area above $20 \AA$ is 1 . Shaded regions show a $68 \%$ confidence interval, computed as described in section S13.

Calculation of the mean end-to-end distance using these modified distributions gives the same scaling exponents for the backbone and spin-label mean end-to-end distances, compared to the means of the unmodified, simulated distributions. The fitted prefactor for the simulated distances is also within uncertainty of the prefactor for the unmodified distributions. The largest difference is in the fitted shift factor, which is 1.8 "monomers" larger for the modified distributions. In other words, removing the states in which the spin labels are oriented towards each other increases their contribution to the effective number of monomers.

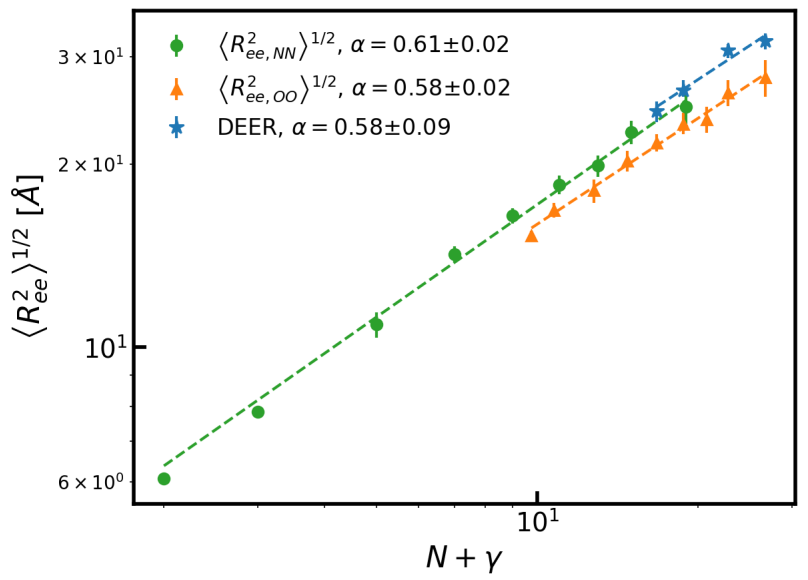

Figure S41: The scaling exponents are fit the same way as reported in the main text. The simulated distances are the mean end-to-end distances for the distributions shown as dotted lines in Fig. S40 along with the corresponding backbone distance distributions (not shown). The backbone end-to-end distances, $\left\langle R_{e e, N N}^{2}\right\rangle^{1 / 2}$ are fit to the power law $b N^{\alpha}$, resulting in a scaling exponent of $\alpha=0.61 \pm 0.02$ and a prefactor of $b=4.2 \pm 0.2$. The spin-label end-to-end 
distances, $\left\langle R_{e e, O o}^{2}\right\rangle^{1 / 2}$, are then fit to a shifted power law, $b(N+\gamma)^{\alpha}$ with $b$ set to 4.2 , yielding scaling exponent of $\alpha=0.58 \pm 0.02$ and a shift factor of $\gamma=7.8$. When the DEER data is fit to $b(N+\gamma)^{\alpha}$ with this shift factor, the resulting scaling exponent is $\alpha=0.58 \pm 0.09$ and the prefactor is $b=4.9 \pm 1.4$.

Another way to remove the contribution of configurations at shorter distances is to simply remove all distances below the DEER optimal region.

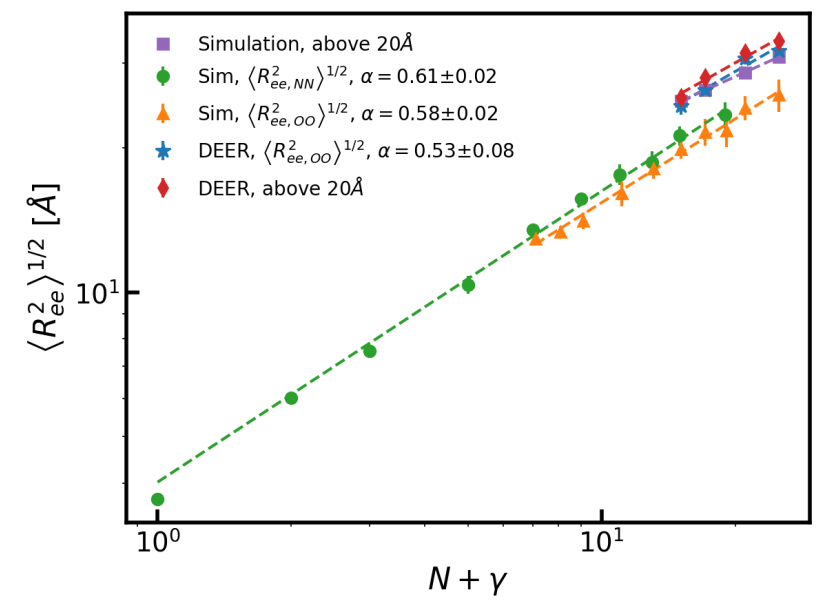

Figure S42: Same as Fig. 7 in the main text but also showing the mean end-to-end distances for the DEER and simulation distributions after cutting off all distances below $20 \AA$. The simulated distances are from the expanded ensemble simulations with the MFTOID forcefield. These are much closer together than the means computed from the full distributions, suggesting that the main source of the inconsistency between the mean distances from DEER and the simulations is the lack of resolution at low end-to-end distances. 


\section{S23. Dependence of predicted distribution on cW-EPR optimal region bounds}

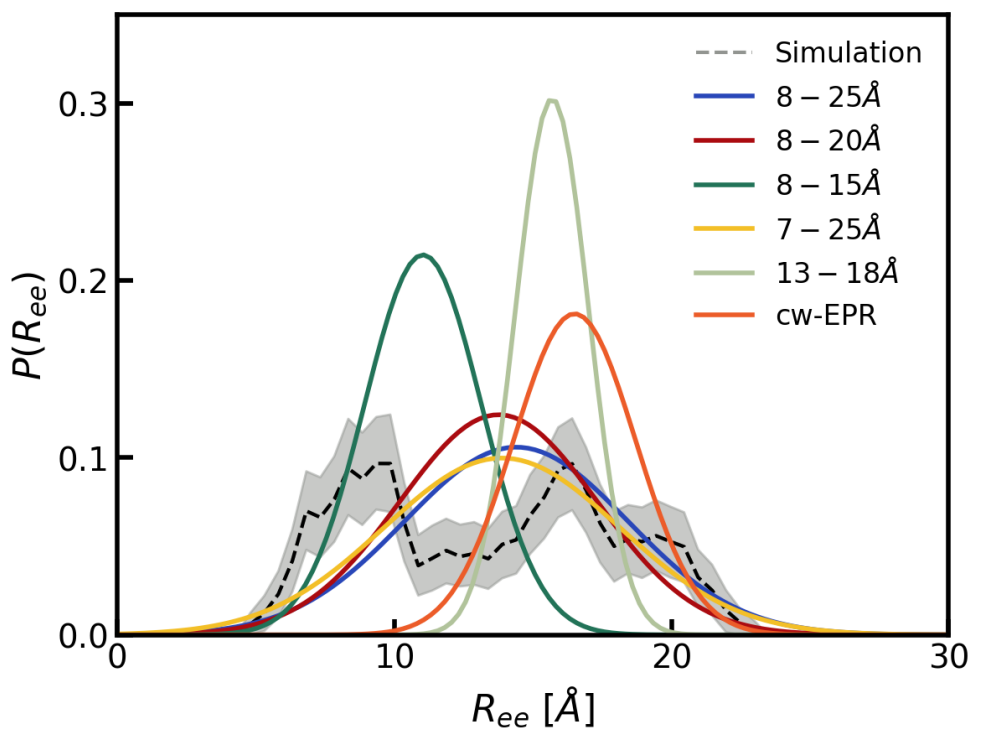

Figure S43: Dependence of predicted distribution on cw-EPR bounds. The cw-EPR calculation approximates the distance distribution as a Gaussian and fits a mean and standard deviation, which should approximately correspond to the mean and standard deviation of the distribution within the optimal region for cw-EPR. The lower bound of the cwEPR region corresponds to the distance at which exchange coupling becomes comparable to dipolar coupling, while the upper bound corresponds to the distance at which broadening becomes negligible. ${ }^{5}$ As both these definitions rely on order-of-magnitude comparisons, there is some uncertainty in the exact bounds for each, which result in variations in the predicted mean and width over the optimal region from the simulated distribution. Here, the black, dashed line shows the simulated end-to-end distribution for the $N=3$ polypeptoid (computed from the expanded ensemble simulation with the MFTOID forcefield), the orange line shows the Gaussian approximation of the end-to-end distance distribution from cw-EPR, and the other colored lines are Gaussian distributions with the same mean and standard deviation of the simulated distribution within various proposed cw-EPR bounds (Table S23).

\begin{tabular}{|l|l|l|l|l|}
\hline $\begin{array}{l}\text { Lower } \\
\text { bound }[\AA]\end{array}$ & $\begin{array}{c}\text { Upper } \\
\text { bound }[\AA]\end{array}$ & \multicolumn{1}{|c|}{$\left\langle\boldsymbol{R}_{\boldsymbol{e}}\right\rangle[\AA]$} & $\boldsymbol{\sigma}_{\boldsymbol{R}_{\boldsymbol{e}}}[\AA]$ & Source \\
\hline 8 & 25 & $14.4(13.9-14.9)$ & 4.0 & $51-53$ \\
\hline 8 & 20 & $13.8(13.3-14.2)$ & 3.6 & $5,54-56$ \\
\hline 8 & 15 & $11.0(10.7-11.4)$ & 2.1 & 5 \\
\hline 7 & 25 & $13.9(13.4-14.4)$ & 4.3 & 57 \\
\hline 13 & 18 & $15.7(15.4-15.9)$ & 1.3 & 58 \\
\hline
\end{tabular}

Table S23: Dependence of predicted mean and standard deviation on cw-EPR bounds. Different definitions of the lower and upper bounds yield different estimates of the mean, $\left\langle R_{e e}\right\rangle$, and standard deviation, $\sigma_{R_{e e}}$, of the simulated distribution (computed from the expanded ensemble simulation of the $N=3$ polypeptoid using the MFTOID forcefield) within those bounds. Numbers inside the parentheses give a $95 \%$ confidence interval of the mean.

Between $8 \AA$ and $25 \AA$, the mean and standard deviation of the simulated distribution are $14.4 \pm$ $0.5 \AA(95 \% \mathrm{CI})$ and $4.0 \AA$, respectively. Both show some deviation from the corresponding cw-EPR measurements, which are $16.5 \pm 0.1 \AA$ and $2.2 \pm 1.2 \AA$, respectively. This discrepancy could result from uncertainty in the estimate of the lower and upper bounds for cw-EPR; while $8-25 \AA$, as first proposed by Rabenstein and Shin, ${ }^{51}$ is the most generally accepted, ${ }^{51-53}$ other bounds have also been reported in the literature ${ }^{5,54,55,57}$ (Table S23), and the predicted mean and standard 
deviation for this relatively narrow distribution are highly sensitive to this choice (Fig. S43). Furthermore, this analysis assumes that all distances within the bounds contribute to the cw-EPR estimates according to their actual population, whereas in reality their contributions may decay close to the boundaries. ${ }^{5}$ 


\section{S24. Visualization of conformational distribution}

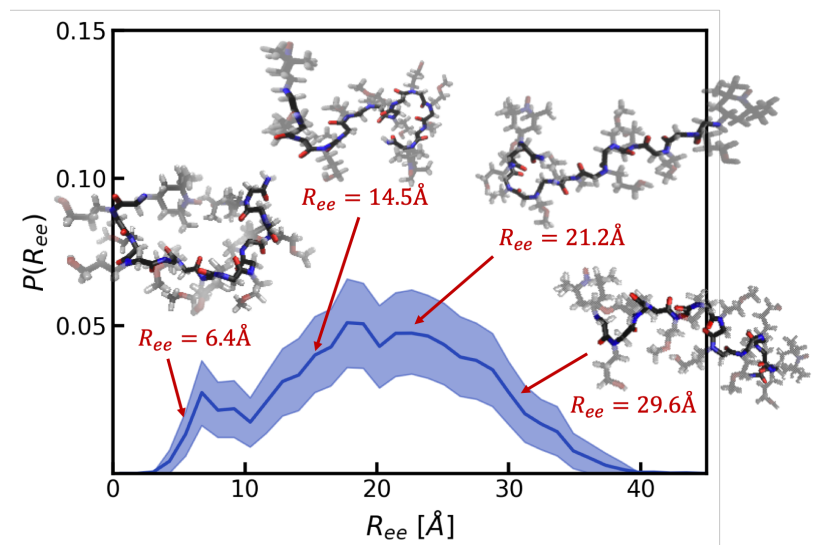

Figure S44: Snapshots from various regions of the end-to-end distribution shows that higher end-to-end distances between the spin-labels correspond with more extended backbone conformations. Snapshots are visualized using VMD. ${ }^{59}$ 


\section{S25. Conformational trends with increasing chain length}

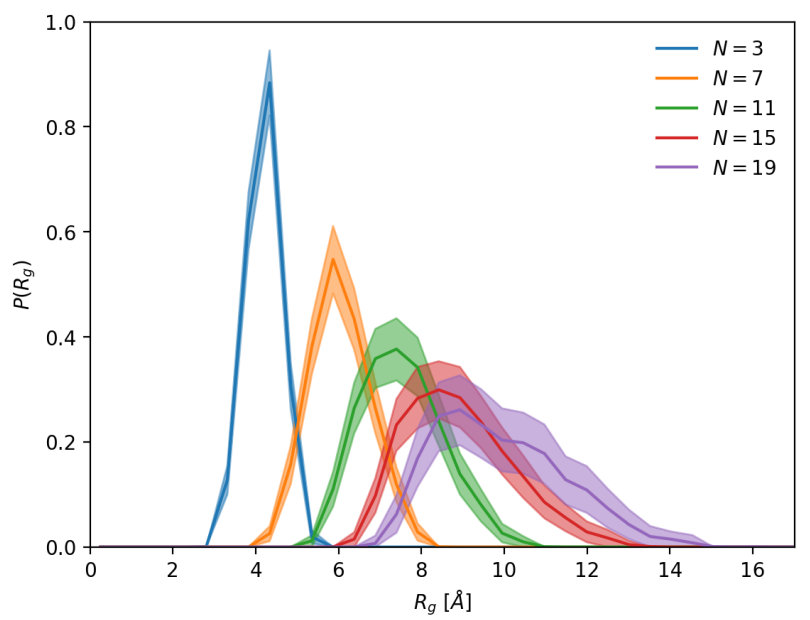

Figure S45: The radii of gyration for each conformation are computed the same way as in Fig. S38 and their distributions are reweighted back to equilibrium in the same way the end-to-end distance distributions are. Similar to the end-to-end distance distributions, the radius of gyration distributions shift to higher distances and become wider with increasing chain length.

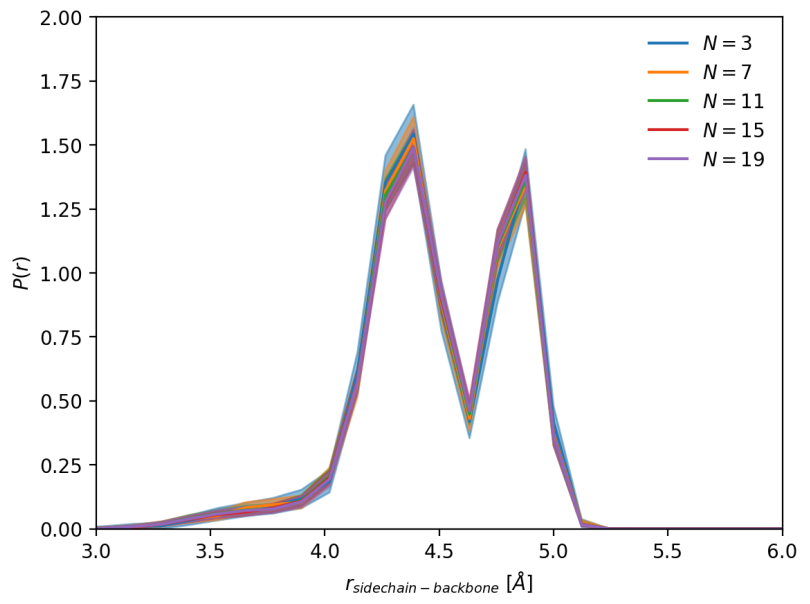

Figure S46: The distribution of distances between the nitrogen atoms on the polypeptoid backbones and the ends of the sidechains (the oxygen in the propanol sidechains and the methyl carbon in the NME sidechains) does not change significantly with chain length.

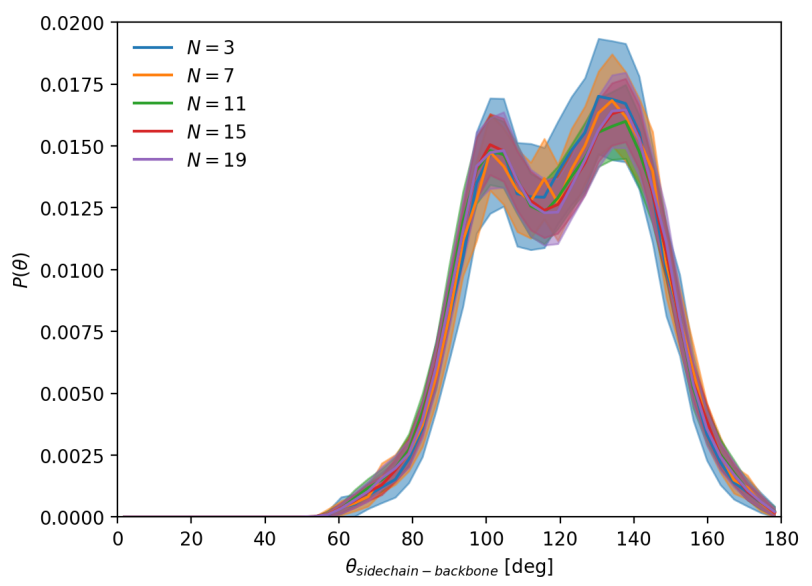


Figure S47: The distribution of angles between a sidechain vector, defined by the backbone nitrogen and end of the sidechain (the oxygen in the propanol sidechains and the methyl carbon in the NME sidechains) and the backbone vector, defined by the backbone nitrogen and carbonyl carbon, does not change significantly with chain length.

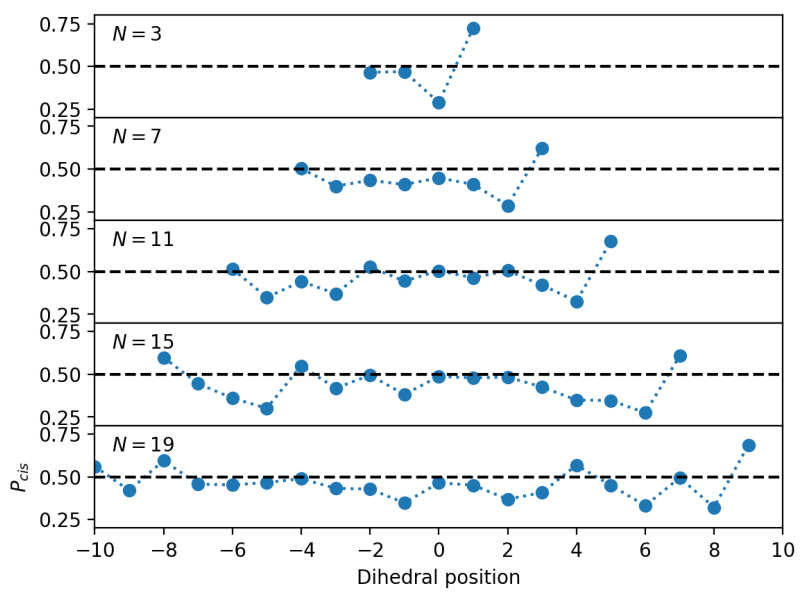

Figure S48: These cis preferences are computed the same way as in Fig. S33. The dashed black lines are at $P_{c i s}=$ 0.5 . As chain length increases, the trends in the cis preferences do not change significantly, with dihedrals in the middle of the polypeptoid remaining close to 0.5 and the last dihedral in the polypeptoid (the one with the nitrogen in the C-terminal spin-label monomer) showing stronger cis preference.

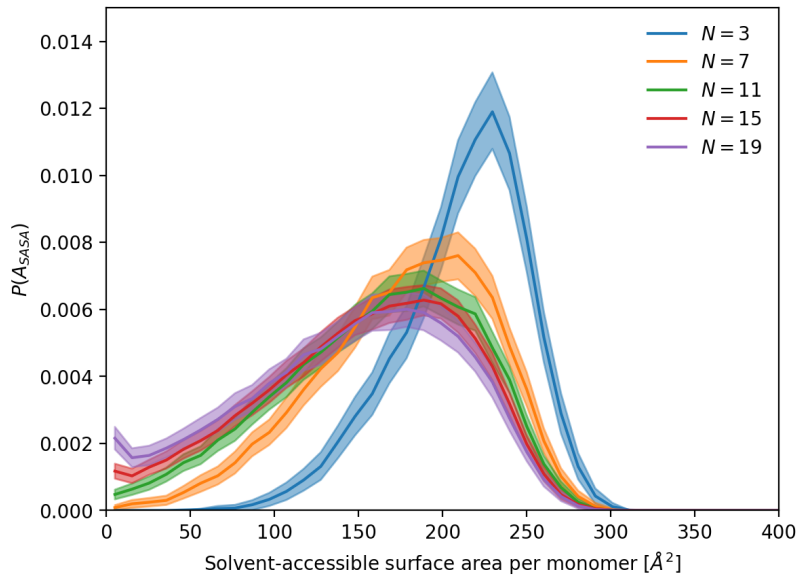

Figure S49: The solvent-accessible surface area for each hydrophilic polypeptoid monomer is computed assigning each atom a radius equal to its Lennard-Jones $\sigma$ parameter and assuming a radius of $1.4 \AA$ for water. The distribution becomes wider and moves to lower areas as chain length increases. 


\section{References}

(1) Figliozzi, G. M.; Goldsmith, R.; Ng, S. C.; Banville, S. C.; Zuckermann, R. N. Synthesis of NSubstituted Glycine Peptoid Libraries. In Methods in Enzymology; Elsevier, 1996; Vol. 267, pp 437-447. https://doi.org/10.1016/S0076-6879(96)67027-X.

(2) Padwa, A.; Rashatasakhon, P.; Ozdemir, A. D.; Willis, J. A Study of Vinyl Radical Cyclization Using $N$-Alkenyl-7-Bromo-Substituted Hexahydroindolinones. The Journal of Organic Chemistry 2005, 70 (2), 519-528. https://doi.org/10.1021/jo048314i.

(3) Schweiger, A.; Jeschke, G. Principles of Pulse Electron Paramagnetic Resonance; Oxford University Press: New York, 2001.

(4) Doll, A.; Qi, M.; Godt, A.; Jeschke, G. CIDME: Short Distances Measured with Long Chirp Pulses. Journal of Magnetic Resonance 2016, 273, 73-82.

https://doi.org/10.1016/j.jmr.2016.10.011.

(5) Banham, J. E.; Baker, C. M.; Ceola, S.; Day, I. J.; Grant, G. H.; Groenen, E. J. J.; Rodgers, C. T.; Jeschke, G.; Timmel, C. R. Distance Measurements in the Borderline Region of Applicability of CW EPR and DEER: A Model Study on a Homologous Series of Spin-Labelled Peptides. Journal of Magnetic Resonance 2008, 191 (2), 202-218.

https://doi.org/10.1016/j.jmr.2007.11.023.

(6) Jeschke, G. DEER Distance Measurements on Proteins. Annual Review of Physical Chemistry 2012, 63 (1), 419-446. https://doi.org/10.1146/annurev-physchem-032511143716.

(7) Sherck, N.; Webber, T.; Brown, D. R.; Keller, T.; Barry, M.; DeStefano, A.; Jiao, S.; Segalman, R. A.; Fredrickson, G. H.; Shell, M. S.; Han, S. End-to-End Distance Probability Distributions of Dilute Poly(Ethylene Oxide) in Aqueous Solution. J. Am. Chem. Soc. 2020, jacs.0c08709. https://doi.org/10.1021/jacs.0c08709.

(8) Altenbach, C. LongDistances; 2019.

(9) Srivastava, M.; Georgieva, E. R.; Freed, J. H. A New Wavelet Denoising Method for Experimental Time-Domain Signals: Pulsed Dipolar Electron Spin Resonance. J. Phys. Chem. A 2017, 121 (12), 2452-2465. https://doi.org/10.1021/acs.jpca.7b00183.

(10) Srivastava, M.; Freed, J. H. Singular Value Decomposition Method to Determine Distance Distributions in Pulsed Dipolar Electron Spin Resonance. J. Phys. Chem. Lett. 2017, 8 (22), 5648-5655. https://doi.org/10.1021/acs.jpclett.7b02379.

(11) Fábregas Ibáñez, L.; Jeschke, G.; Stoll, S. DeerLab: A Comprehensive Software Package for Analyzing Dipolar Electron Paramagnetic Resonance Spectroscopy Data. Magn. Reson. 2020, 1 (2), 209-224. https://doi.org/10.5194/mr-1-209-2020.

(12) Easley, A. D.; Vukin, L. M.; Flouda, P.; Howard, D. L.; Pena, J. L.; Lutkenhaus, J. L. Nitroxide Radical Polymer-Solvent Interactions and Solubility Parameter Determination.

Macromolecules 2020, 53 (18), 7997-8008.

https://doi.org/10.1021/acs.macromol.0c01739.

(13) Altenbach, C. ShortDistances; 2014.

(14) Hanwell, M. D.; Curtis, D. E.; Lonie, D. C.; Vandermeersch, T.; Zurek, E.; Hutchison, G. R. Avogadro: An Advanced Semantic Chemical Editor, Visualization, and Analysis Platform. J Cheminform 2012, 4 (1), 17. https://doi.org/10.1186/1758-2946-4-17.

(15) Gaussian 16, Revision C.01; Gaussian, Inc.: Wallingford CT, 2016. 
(16) AMBER 2016; University of California, San Francisco, 2016.

(17) Prakash, A.; Baer, M. D.; Mundy, C. J.; Pfaendtner, J. Peptoid Backbone Flexibilility Dictates Its Interaction with Water and Surfaces: A Molecular Dynamics Investigation.

Biomacromolecules 2018, 19 (3), 1006-1015.

https://doi.org/10.1021/acs.biomac.7b01813.

(18) Mirijanian, D. T.; Mannige, R. V.; Zuckermann, R. N.; Whitelam, S. Development and Use of an Atomistic CHARMM-Based Forcefield for Peptoid Simulation. J. Comput. Chem. 2014, 35 (5), 360-370. https://doi.org/10.1002/jcc.23478.

(19) Vorobyov, I.; Anisimov, V. M.; Greene, S.; Venable, R. M.; Moser, A.; Pastor, R. W.; MacKerell, A. D. Additive and Classical Drude Polarizable Force Fields for Linear and Cyclic Ethers. J. Chem. Theory Comput. 2007, 3 (3), 1120-1133. https://doi.org/10.1021/ct600350s.

(20) Sezer, D.; Freed, J. H.; Roux, B. Parametrization, Molecular Dynamics Simulation, and Calculation of Electron Spin Resonance Spectra of a Nitroxide Spin Label on a Polyalanine a-Helix. J. Phys. Chem. B 2008, 112 (18), 5755-5767. https://doi.org/10.1021/jp711375x.

(21) Stendardo, E.; Pedone, A.; Cimino, P.; Cristina Menziani, M.; Crescenzi, O.; Barone, V. Extension of the AMBER Force-Field for the Study of Large Nitroxides in Condensed Phases: An Ab Initio Parameterization. Phys. Chem. Chem. Phys. 2010, 12 (37), 11697. https://doi.org/10.1039/c001481h.

(22) MacKerell, A. D.; Bashford, D.; Bellott, M.; Dunbrack, R. L.; Evanseck, J. D.; Field, M. J.; Fischer, S.; Gao, J.; Guo, H.; Ha, S.; Joseph-McCarthy, D.; Kuchnir, L.; Kuczera, K.; Lau, F. T. K.; Mattos, C.; Michnick, S.; Ngo, T.; Nguyen, D. T.; Prodhom, B.; Reiher, W. E.; Roux, B.; Schlenkrich, M.; Smith, J. C.; Stote, R.; Straub, J.; Watanabe, M.; Wiórkiewicz-Kuczera, J.; Yin, D.; Karplus, M. All-Atom Empirical Potential for Molecular Modeling and Dynamics Studies of Proteins. J. Phys. Chem. B 1998, 102 (18), 3586-3616. https://doi.org/10.1021/jp973084f.

(23) Vanommeslaeghe, K.; Hatcher, E.; Acharya, C.; Kundu, S.; Zhong, S.; Shim, J.; Darian, E.; Guvench, O.; Lopes, P.; Vorobyov, I.; Mackerell, A. D. CHARMM General Force Field: A Force Field for Drug-like Molecules Compatible with the CHARMM All-Atom Additive Biological Force Fields. J. Comput. Chem. 2009, NA-NA. https://doi.org/10.1002/jcc.21367.

(24) Vanommeslaeghe, K.; MacKerell, A. D. Automation of the CHARMM General Force Field (CGenFF) I: Bond Perception and Atom Typing. J. Chem. Inf. Model. 2012, 52 (12), 31443154. https://doi.org/10.1021/ci300363c.

(25) Vanommeslaeghe, K.; Raman, E. P.; MacKerell, A. D. Automation of the CHARMM General Force Field (CGenFF) II: Assignment of Bonded Parameters and Partial Atomic Charges. J. Chem. Inf. Model. 2012, 52 (12), 3155-3168. https://doi.org/10.1021/ci3003649.

(26) Weiser, L. J.; Santiso, E. E. A CGenFF-based Force Field for Simulations of Peptoids with Both Cis and Trans Peptide Bonds. J Comput Chem 2019, 40 (22), 1946-1956. https://doi.org/10.1002/jcc.25850.

(27) Mackerell, A. D.; Feig, M.; Brooks, C. L. Extending the Treatment of Backbone Energetics in Protein Force Fields: Limitations of Gas-Phase Quantum Mechanics in Reproducing Protein Conformational Distributions in Molecular Dynamics Simulations. J. Comput. Chem. 2004, 25 (11), 1400-1415. https://doi.org/10.1002/jcc.20065. 
(28) Berendsen, H. J. C.; Grigera, J. R.; Straatsma, T. P. The Missing Term in Effective Pair Potentials. J. Phys. Chem. 1987, 91 (24), 6269-6271.

https://doi.org/10.1021/j100308a038.

(29) Jakalian, A.; Jack, D. B.; Bayly, C. I. Fast, Efficient Generation of High-Quality Atomic Charges. AM1-BCC Model: II. Parameterization and Validation. J. Comput. Chem. 2002, 23 (16), 1623-1641. https://doi.org/10.1002/jcc.10128.

(30) Bayly, C. I.; Cieplak, P.; Cornell, W.; Kollman, P. A. A Well-Behaved Electrostatic Potential Based Method Using Charge Restraints for Deriving Atomic Charges: The RESP Model. J. Phys. Chem. 1993, 97 (40), 10269-10280. https://doi.org/10.1021/j100142a004.

(31) Prakash, A.; Baer, M. D.; Mundy, C. J.; Pfaendtner, J. Peptoid Backbone Flexibilility Dictates Its Interaction with Water and Surfaces: A Molecular Dynamics Investigation.

Biomacromolecules 2018, 19 (3), 1006-1015. https://doi.org/10.1021/acs.biomac.7b01813.

(32) Weiser, L. J.; Santiso, E. E. A CGenFF-based Force Field for Simulations of Peptoids with Both Cis and Trans Peptide Bonds. J Comput Chem 2019, 40 (22), 1946-1956. https://doi.org/10.1002/jcc.25850.

(33) Edwards, T. H.; Stoll, S. A Bayesian Approach to Quantifying Uncertainty from Experimental Noise in DEER Spectroscopy. Journal of Magnetic Resonance 2016, 270, 8797. https://doi.org/10.1016/j.jmr.2016.06.021.

(34) Kaminker, R.; Kaminker, I.; Gutekunst, W. R.; Luo, Y.; Lee, S.; Niu, J.; Han, S.; Hawker, C. J. Tuning Conformation and Properties of Peptidomimetic Backbones through Dual $\mathrm{N} / \mathrm{C}_{\alpha}-$ Substitution. Chem. Commun. 2018, 54 (41), 5237-5240. https://doi.org/10.1039/C8CC01356J.

(35) Sui, Q.; Borchardt, D.; Rabenstein, D. L. Kinetics and Equilibria of Cis/Trans Isomerization of Backbone Amide Bonds in Peptoids. J. Am. Chem. Soc. 2007, 129 (39), 12042-12048. https://doi.org/10.1021/ja0740925.

(36) Mukherjee, S.; Zhou, G.; Michel, C.; Voelz, V. A. Insights into Peptoid Helix Folding Cooperativity from an Improved Backbone Potential. J. Phys. Chem. B 2015, 119 (50), 15407-15417. https://doi.org/10.1021/acs.jpcb.5b09625.

(37) Nguyen, H.; Roe, D. R.; Simmerling, C. Improved Generalized Born Solvent Model Parameters for Protein Simulations. J. Chem. Theory Comput. 2013, 9 (4), 2020-2034. https://doi.org/10.1021/ct3010485.

(38) van der Spoel, D.; Berendsen, H. J. Molecular Dynamics Simulations of Leu-Enkephalin in Water and DMSO. Biophysical Journal 1997, 72 (5), 2032-2041. https://doi.org/10.1016/S0006-3495(97)78847-7.

(39) Tan, Z. Optimally Adjusted Mixture Sampling and Locally Weighted Histogram Analysis. Journal of Computational and Graphical Statistics 2017, 26 (1), 54-65. https://doi.org/10.1080/10618600.2015.1113975.

(40) Shirts, M. R.; Chodera, J. D. Statistically Optimal Analysis of Samples from Multiple Equilibrium States. The Journal of Chemical Physics 2008, 129 (12), 124105. https://doi.org/10.1063/1.2978177.

(41) Chodera, J. D.; Swope, W. C.; Pitera, J. W.; Seok, C.; Dill, K. A. Use of the Weighted Histogram Analysis Method for the Analysis of Simulated and Parallel Tempering 
Simulations. J. Chem. Theory Comput. 2007, 3 (1), 26-41.

https://doi.org/10.1021/ct0502864.

(42) Shirts, M. R.; Chodera, J. D. Statistically Optimal Analysis of Samples from Multiple Equilibrium States. The Journal of Chemical Physics 2008, 129 (12), 124105. https://doi.org/10.1063/1.2978177.

(43) Chodera, J. D.; Swope, W. C.; Pitera, J. W.; Seok, C.; Dill, K. A. Use of the Weighted Histogram Analysis Method for the Analysis of Simulated and Parallel Tempering Simulations. J. Chem. Theory Comput. 2007, 3 (1), 26-41. https://doi.org/10.1021/ct0502864.

(44) Levina, E.; Bickel, P. The Earth Mover's Distance Is the Mallows Distance: Some Insights from Statistics. In Proceedings Eighth IEEE International Conference on Computer Vision. ICCV 2001; IEEE Comput. Soc: Vancouver, BC, Canada, 2001; Vol. 2, pp 251-256. https://doi.org/10.1109/ICCV.2001.937632.

(45) Onufriev, A. V.; Izadi, S. Water Models for Biomolecular Simulations: Water Models for Biomolecular Simulations. WIREs Comput Mol Sci 2018, 8 (2), e1347. https://doi.org/10.1002/wcms.1347.

(46) Izadi, S.; Anandakrishnan, R.; Onufriev, A. V. Building Water Models: A Different Approach. J. Phys. Chem. Lett. 2014, 5 (21), 3863-3871. https://doi.org/10.1021/jz501780a.

(47) Knight, A. S.; Zhou, E. Y.; Francis, M. B.; Zuckermann, R. N. Sequence Programmable Peptoid Polymers for Diverse Materials Applications. Adv. Mater. 2015, 27 (38), 56655691. https://doi.org/10.1002/adma.201500275.

(48) Schäfer, L. Excluded Volume Effects in Polymer Solutions; Springer Berlin Heidelberg: Berlin, Heidelberg, 1999. https://doi.org/10.1007/978-3-642-60093-7.

(49) Zhao, M.; Sampath, J.; Alamdari, S.; Shen, G.; Chen, C.-L.; Mundy, C. J.; Pfaendtner, J.; Ferguson, A. L. A MARTINI-Compatible Coarse-Grained Model for the Mesoscale Simulation of Peptoids. J. Phys. Chem. B 2020, acs.jpcb.0c04567. https://doi.org/10.1021/acs.jpcb.0c04567.

(50) Kabsch, W. A Solution for the Best Rotation to Relate Two Sets of Vectors. Acta Cryst A 1976, 32 (5), 922-923. https://doi.org/10.1107/S0567739476001873.

(51) Rabenstein, M. D.; Shin, Y. K. Determination of the Distance between Two Spin Labels Attached to a Macromolecule. Proceedings of the National Academy of Sciences 1995, 92 (18), 8239-8243. https://doi.org/10.1073/pnas.92.18.8239.

(52) Cooke, J. A.; Brown, L. J. Distance Measurements by Continuous Wave EPR Spectroscopy to Monitor Protein Folding. In Protein Folding, Misfolding, and Disease; Hill, A. F., Barnham, K. J., Bottomley, S. P., Cappai, R., Eds.; Methods in Molecular Biology; Humana Press: Totowa, NJ, 2011; Vol. 752, pp 73-96. https://doi.org/10.1007/978-1-60327-2230_6.

(53) Liu, Y.-S.; Sompornpisut, P.; Perozo, E. Structure of the KcsA Channel Intracellular Gate in the Open State. Nat. Struct Biol. 2001, 8 (10), 883-887. https://doi.org/10.1038/nsb1001883.

(54) Persson, M.; Harbridge, J. R.; Hammarström, P.; Mitri, R.; Mårtensson, L.-G.; Carlsson, U.; Eaton, G. R.; Eaton, S. S. Comparison of Electron Paramagnetic Resonance Methods to Determine Distances between Spin Labels on Human Carbonic Anhydrase II. Biophysical Journal 2001, 80 (6), 2886-2897. https://doi.org/10.1016/S0006-3495(01)76254-6. 
(55) Brown, L. J.; Sale, K. L.; Hills, R.; Rouviere, C.; Song, L.; Zhang, X.; Fajer, P. G. Structure of the Inhibitory Region of Troponin by Site Directed Spin Labeling Electron Paramagnetic Resonance. Proceedings of the National Academy of Sciences 2002, 99 (20), 12765-12770. https://doi.org/10.1073/pnas.202477399.

(56) Chiang, Y.-W.; Zheng, T.-Y.; Kao, C.-J.; Horng, J.-C. Determination of Interspin Distance Distributions by Cw-ESR Is a Single Linear Inverse Problem. Biophysical Journal 2009, 97 (3), 930-936. https://doi.org/10.1016/j.bpj.2009.05.030.

(57) Zhang, F.; Chen, Y.; Kweon, D.-H.; Kim, C. S.; Shin, Y.-K. The Four-Helix Bundle of the Neuronal Target Membrane SNARE Complex Is Neither Disordered in the Middle nor Uncoiled at the C-Terminal Region. J. Biol. Chem. 2002, 277 (27), 24294-24298. https://doi.org/10.1074/jbc.M201200200.

(58) Gunnar Jeschke. Introduction to Electron Paramagnetic Resonance Spectroscopy.

(59) Humphrey, W.; Dalke, A.; Schulten, K. VMD: Visual Molecular Dynamics. Journal of Molecular Graphics 1996, 14 (1), 33-38. https://doi.org/10.1016/0263-7855(96)00018-5. 